\title{
SENSOR INTEGRATION FOR Low-Cost CRASH AvoIDANCE
}

\author{
A Thesis \\ presented to \\ the Faculty of California Polytechnic State University, \\ San Luis Obispo
}

\author{
In Partial Fulfillment \\ of the Requirements for the Degree \\ Master of Science in Mechanical Engineering
}

by

Stephane M. Roussel

October 2009 
(C) 2009

Stephane M. Roussel

ALL RIGHTS RESERVED 


\section{COMMITTEE MEMBERSHIP}

TITLE:

Project

AUTHOR:

DATE SUBMITTED:

COMMITTEE CHAIR:

COMMITTEE MEMBER:

COMMITTEE MEMBER:

COMMITTEE MEMBER
Sensor Integration for Low-Cost Crash Avoidance

Stephane M. Roussel

October 18, 2009

Charles Birdsong Ph.D., Associate Professor

Peter Schuster Ph.D., Associate Professor

Hemanth Porumamilla Ph.D., Assistant Professor

Christopher Clark Ph.D., Assistant Professor 


\section{ABSTRACT \\ Sensor Integration for Low-Cost Crash Avoidance Project \\ Stephane Roussel}

This report is a summary of the development of sensor integration for low-cost crash avoidance for over-land commercial trucks. The goal of the project was to build and test a system composed of low-cost commercially available sensors arranged on a truck trailer to monitor the environment around the truck. The system combines the data from each sensor to increase the reliability of the sensor using a probabilistic data fusion approach. A combination of ultrasonic and magnetoresistive sensors was used in this study. In addition, Radar and digital imaging were investigated as reference signals and possible candidates for additional sensor integration. However, the primary focus of this work is the integration of the ultrasonic and magnetoresistive sensors.

During the investigation the individual sensors were evaluated for their use in the system. This included communication with vendors and lab and field testing. In addition, the sensors were modeled using an analytical mathematical model to help understand and predict the sensor behavior. Next, an algorithm was developed to fuse the data from the individual sensors. A probabilistic approach was used based on Bayesian filtering with a prediction-correction algorithm. Sensor fusion was implemented using joint a probability algorithm. The output of the system is a prediction of the likelihood of the presence of a vehicle in a given region near the host truck trailer. The algorithm was demonstrated on the fusion of an ultrasonic sensor and a magnetic sensor. Testing was conducted using both a light pickup truck and also with a class 8 truck. Various scenarios were evaluated to determine the system performance. These included vehicles passing the host truck 
from behind and the host truck passing vehicles. Also scenarios were included to test the system at distinguishing other vehicles from objects that are not vehicles such as sign posts, walls or railroads that could produce electronic signals similar to those of vehicles and confuse the system. The test results indicate that the system was successful at predicting the presence and absence of vehicles and also successful at eliminating false positives from objects that are not vehicles with overall accuracy ranging from 90 to $100 \%$ depending on the scenario. Some additional improvements in the performance are expected with future improvements in the algorithm discussed in the report.

The report includes a discussion of the mapping of the algorithm output with the implementation of current and future safety and crash avoidance technologies based on the level of confidence of the algorithm output and the seriousness of the impending crash scenario. For example, irreversible countermeasures such as firing an airbag or engaging the brakes should only be initiated if the confidence of the signal is very high, while reversible countermeasures such as warnings to the driver or nearby vehicles can be initiated with a relatively lower confidence.

The results indicate that the system shows good potential as a low cost alternative to competing systems which require multiple, high cost sensors. Truck fleet operators will likely adopt technology only if the costs are justified by reduced damage and insurance costs, therefore developing an effective crash avoidance system at a low cost is required for the technology to be adopted on a large scale. 


\section{ACKNOWLEDGEMENTS}

This work would not have been possible without the support and encouragement of my thesis committee. I first started working on this project with Dr. Charles Birdsong and Dr. Peter Schuster. These two have provided continuous support and guidance throughout this work. Soon after, Dr. Hemanth Porumamilla joined this group and since has been a mentor and continually challenged me to do my best in both academia and industry. Finally, I want to thank Dr. Christopher Clark for his insight and support in probabilistic methods. Without his contributions this project would not have been possible. I am thankful for the support and guidance of this committee has given me over the time span of this project.

I would also like to acknowledge the contributions of research assistants and friends. Mario Garcia, Joey Marino, and Steve Johnson provided the software and hardware support throughout this project. Their ingenuity and hard work is of an unmatched caliber and was the foundation of this project. I would also like to thank my colleagues and friends from the graduate lab. Chris Biddy, Tom Gwon, and Raghav Pancholy are the few that know how to keep spirits up when times are rough. No matter the situation, I know I can always turn to this group for their insight and advice. It has been a pleasure to work and be in the company of this group.

I cannot end without thanking my family and close friends, whose constant encouragement and support I have relied on through my academic career. They continually inspire me and give me the drive necessary to succeed. It is to them that I dedicate this work. 


\section{TABLE OF CONTENTS}

LIST OF FIGURES ............................................................................................................................. ix

LIST OF TABLES......................................................................................................

CHAPTERS.......................................................................................................................... xi

1. PROJECT BACKGROUND .................................................................................................. 1

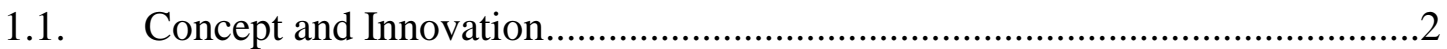

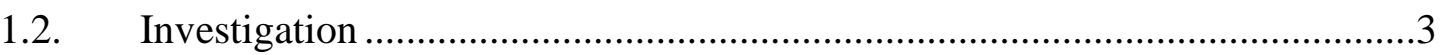

2. SENSOR TESTING AND MODELING .......................................................................... 5

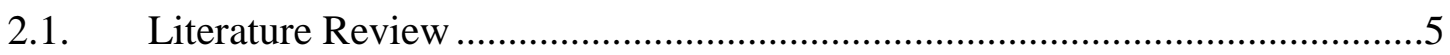

2.1.1. Accident Data 5

2.1.2. State-of-the-art Sensors 6

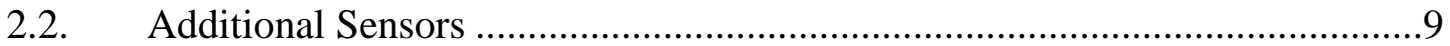

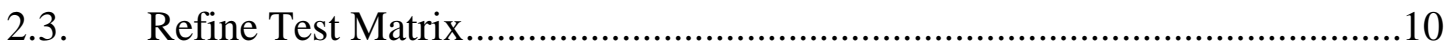

2.4. Sensor Modeling ...............................................................................11

2.4.1. Magnetic Sensor 11

2.4.2. Ultrasonic Sensor 21

2.4.3. Computer Vision Sensor Modeling 24

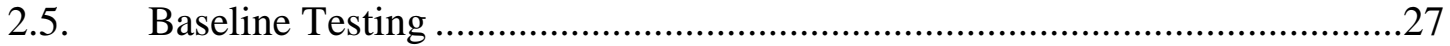

2.5.1. Magnetic Sensor Testing 27

2.5.2. Radar Sensor Testing 29

2.5.3. Ultrasonic Sensor Testing 32

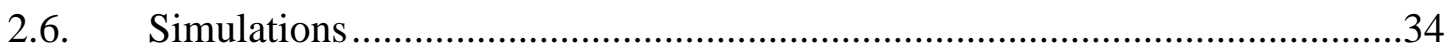

3. VEHICLE DETECTION ALGORITHM ..........................................................................38

3.1. Algorithm Development.........................................................................

3.1.1. Basic Concepts in Probability 38

3.1.2. Bayesian Filtering 41

3.1.3. Bayesian Filter Algorithm 42

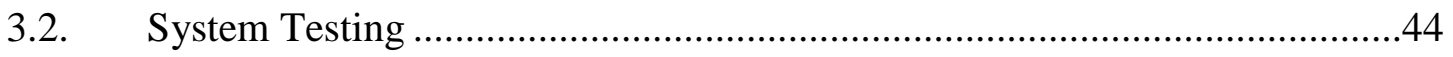

3.2.1. Data Collection 44

3.2.2. Statistical Sensor Modeling (Individual Sensors) 45

3.2.3. Bayesian Filter Results (Individual Sensors) 49

3.2.4. Sensor Fusion 51 
3.2.5. Full Scale Testing 53

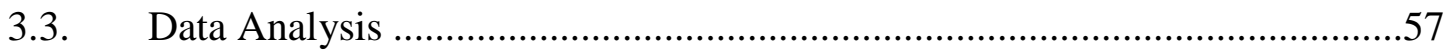

3.3.1. Bayes Filter Algorithm Performance 57

3.3.2. Filter Performance 60

3.3.3. Improving Filter Performance 67

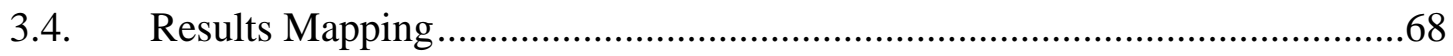

3.4.1. Improving Truck Driver Awareness 69

3.4.2. Improve Nearby Drivers’ Awareness 70

3.4.3. Preventing Risky Actions 71

3.4.4. Taking Preventative Actions $\quad 72$

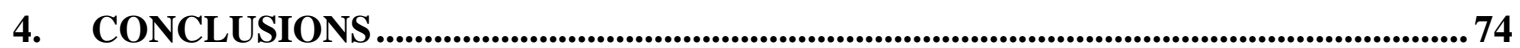

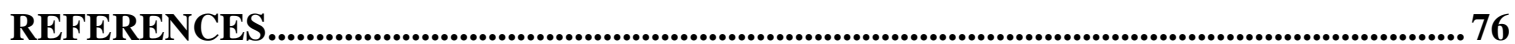




\section{LIST OF FIGURES}

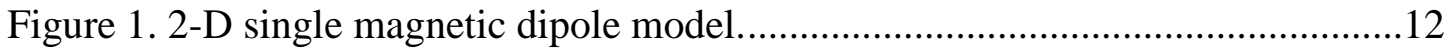

Figure 2. Magnetic dipole in 3-D space ........................................................... 14

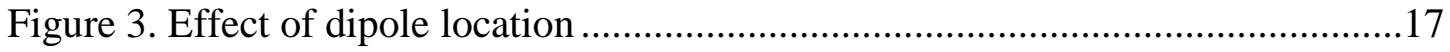

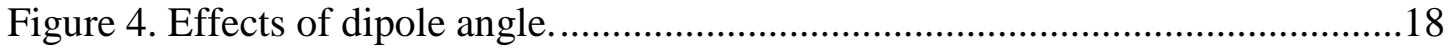

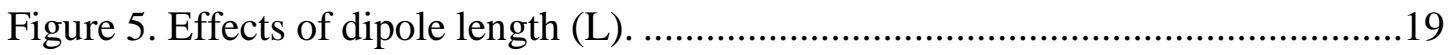

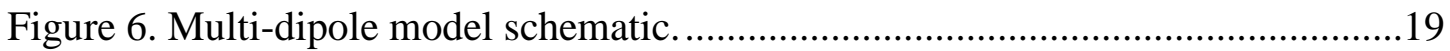

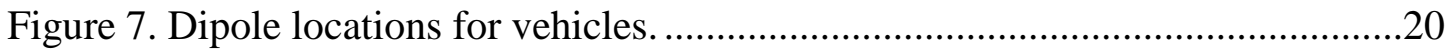

Figure 8. Minimum object sizes for side-mounted ultrasonic sensor return signal.....23

Figure 9. Coverage zone for side-mounted ultrasonic sensors. .............................24

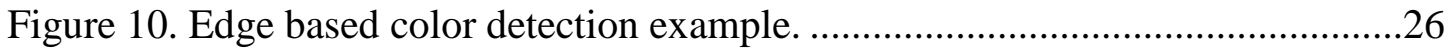

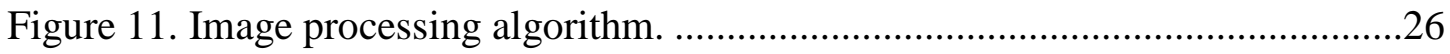

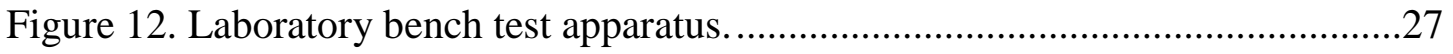

Figure 13. Magnetic sensor bench test results. ................................................28

Figure 14. Magnetic sensor road test configuration.............................................29

Figure 15. Magnetic sensor road test result. ......................................................29

Figure 16. Schematic of radar azimuth and elevation angle measurements...............30

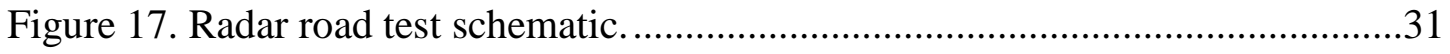

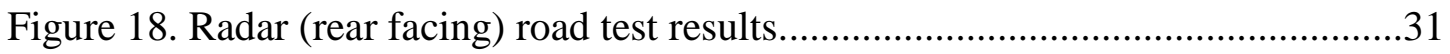

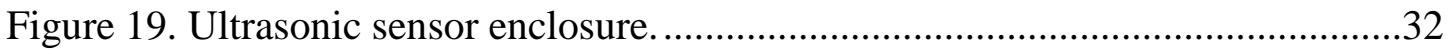

Figure 20. Ultrasonic sensor beam characteristic. ............................................... 33

Figure 21. Ultrasonic sensor mobile test setup................................................... 34

Figure 22. Comparison of simple dipole model experiment and simulation ................35

Figure 23. Magnetic threshold for object discrimination for simple dipoles...............36

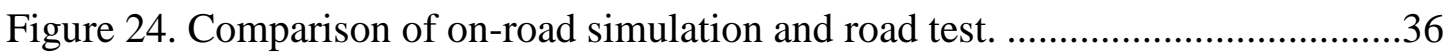


Figure 25. Magnetic threshold for object discrimination for vehicles.

Figure 26. Joint likelihood of two sensors [22]............................................... 40

Figure 27. Raw ultrasonic data with noise...........................................................42

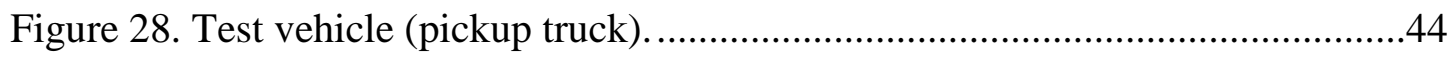

Figure 29. Sensor data for vehicle passing scenario. ............................................45

Figure 30. Ultrasonic sensor belief distribution when vehicle present.....................46

Figure 31. Ultrasonic sensor belief distribution when vehicle absent. .....................47

Figure 32. Magnetic sensor belief distribution when vehicle present. .......................48

Figure 33. Ultrasonic sensor raw data and predictive models. ...............................49

Figure 34. Bayesian filter algorithm (individual sensor) .....................................50

Figure 35. Likelihood of vehicle presence (ultrasonic sensor). ...............................50

Figure 36. Likelihood of vehicle presence (magnetic sensor)..................................51

Figure 37. Bayesian filter algorithm (multiple sensors). ......................................51

Figure 38. Joint likelihood of vehicle presence. ..................................................52

Figure 39. Joint likelihood of a non-metallic object. ..........................................52

Figure 40. Equipment housing for road testing. ..................................................54

Figure 41. Carriage for equipment housing. .....................................................54

Figure 42. Equipment housing and carriage on large truck...................................55

Figure 43. Sensor placement on large truck.......................................................55

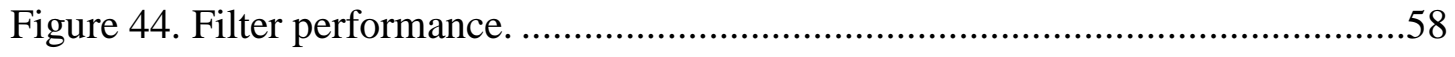

Figure 45. Two passenger vehicles test using developed and tuned senor models......62

Figure 46. Trash containers test using developed and tuned sensor models...............63

Figure 47. Railroad tracks test using developed sensor model...............................64

Figure 48. Metal building test using developed and tuned sensor model. ..................65

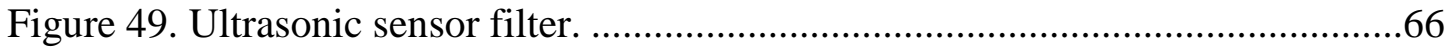




\section{LIST OF TABLES}

TABLE 1. Variables and equations to determine ultrasonic sensor placement..........24

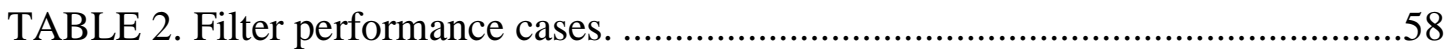

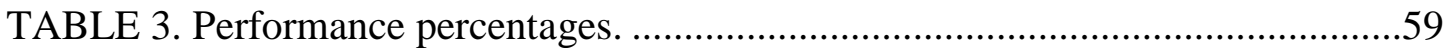

TABLE 4. Example performance results table.........................................................60

TABLE 5. Filter performance while passing two passenger vehicles. ......................62

TABLE 6. Filter performance while passing a vehicle and trash containers..............63

TABLE 7. Filter performance while driving over railroad tracks (high magnetic influence).

TABLE 8. Filter performance while driving over railroad tracks (high magnetic signature and high range influence) .........................................................66 


\section{PROJECT BACKGROUND}

The project is aimed at developing a sensing system for Class 8 trucks that detects objects around the vehicle, discriminates between object types, and determines object threat levels. This system will provide data to enable multiple accident avoidance countermeasures, such as:

- Support decision-making in engaging on-board safety systems (e.g. airbags)

- Warn driver of potential threat objects in projected path (audible, visual, or haptic)

- Prevent drivers from engaging in risky maneuvers (e.g. turning across a vehicle's path)

- Perform preventative measures to avoid accidents (e.g. braking or stabilizing)

- Perform protective measures to reduce accident severity (e.g. braking or deployments)

To achieve these goals, this project will focus on developing a sensor integration system to gather and process data from a wide selection of low-cost exterior sensors. By taking advantage of multiple sensors, we can fill the gaps in coverage and avoid the limitations in detection that a single type of sensor inevitably has. In addition, we can use the different sensor results returned from the same object to improve our object discrimination and threat decisions.

The product will be a system consisting of multiple sensors connected by one or more digital computer processors. The computers will host a software algorithm which handles communication from the sensors, signal processing and filtering, and data fusion. The output of the algorithm will be capable of providing feedback to the driver and triggering safety device deployment. Result mapping will provide a mapping between sensor output and recommended actions triggered within the vehicle. Note that implementing and testing safety devices is outside the scope of this project. 


\subsection{CONCEPT AND INNOVATION}

This research project makes use of low-cost readily available sensors to detect objects outside the commercial vehicle. Because the data from these individual sensors may not be reliable enough to make irreversible decisions, a critical data fusion step must be taken. Data from the multiple sensors will be integrated, or 'fused' together to create a situational awareness within the system that is far greater than the individual sensors can provide, at a price far less than a new customdesigned sensor.

Although not all accidents can be eliminated, a properly functioning detection system (the aim of this project) should be capable of predicting more than $90 \%$ of the potential accident situations. Many of these can be avoided by warning the truck driver to avoid maneuvers (such as lane changes or turns), or with an appropriate warning system for other drivers at the side or the rear of the truck. In addition, new automated systems, such as a resistance to turning, or automated braking, may be used to prevent further crashes.

The implementation of these systems in commercial vehicles should result in an immediate reduction in the number and severity of crashes. We anticipate the benefits from internal warnings and/or automated responses should be permanent. However, without further migration of the technology into personal use vehicles as well, any exterior warnings may prove of limited benefit as other drivers become used to the warnings and resume more risky behaviors. Fortunately, the sensor integration approach proposed in this research applies equally well to object detection for passenger cars. Once a reliable low-cost system has been demonstrated in commercial vehicles, it should not take long to migrate into passenger cars, thereby providing additional life-saving benefits. 


\subsection{INVESTIGATION}

The investigation is organized into two stages: Sensor Testing and Modeling and Vehicle Detection Algorithm. Sensor Testing and Modeling consists of literature review and acquiring and testing candidate sensors. Vehicle Detection Algorithm consists of algorithm development, system testing, data analysis, result mapping and final documentation and review.

\section{Sensor Testing \& Modeling}

- Literature Review: Reviewed work done by others and identified additional sensors for this application.

- Additional Sensors: The literature review identified additional promising sensors that were considered for integration into the final system. This task involved identifying manufacturers for these sensors, contacting manufacturers, negotiating equipment prices or donations, and ordering parts.

- Refine Test Matrix: Another outcome of the literature review was more data on sensor limitations. Together with input from sensor suppliers, in task 4 these limitations were used to refine existing test matrices for previously obtained sensors and define test protocols for the new sensors.

- Modeling: To generate the most information in the shortest time, a set of mathematical models for each sensor was developed. These models predict the anticipated output from the sensors under a variety of circumstances. The modeling will aid in the simulation and algorithm development. Modeling included effects of sensor range, noise, sampling rate, etc. Sensor target models were developed to test the system with targets of different size, texture, distance, velocity, acceleration, etc. The objective is to use the modeling to test proposed algorithms to process sensor data and predict a crash event.

- Baseline Testing: The goal of the initial testing was to confirm the validity of the mathematical models and identify the key strengths and weaknesses of each sensor. In addition, experience with these tests enabled the final product confirmation tests to be more completely defined.

- Simulations: After the models were validated by baseline testing of the sensors, certain design parameters (e.g. size, locations, and power) were varied to define 
the most robust configuration under a variety of sensing scenarios. Simulations were also used to confirm individual sensor's strengths and weaknesses and determine a fit between the proposed sensors.

\section{Vehicle Detection Algorithm}

- Algorithm Development: Develop preliminary algorithms for integrating ('fusing') sensors into a complete vehicle collision avoidance sensing system.

- System Testing: Completed physical testing of the sensor configurations identified in Task 7 together with the integration algorithm defined in Task 9. These tests consisted of lab bench tests, static traffic tests, and dynamic traffic tests on subject vehicles.

- Data Analysis: The data collected during system testing was analyzed to determine the potential for the sensor system to perform collision risk predictions

- Results Mapping: After reviewing the results of the sensor and algorithm tests, the team identified and evaluated a set of accident avoidance countermeasures that might be triggered by this system. Based on sensor performance, connections were proposed between specific system predictions and potential countermeasures. These connections are intended to be used by potential implementers to decide how to use the sensing system to effect changes in the real-world traffic environment.

Once a set of options has been identified, these will be associated with particular outputs from the sensor algorithm. For example, a high probability of crash near the vehicle center might trigger pre-crash braking to reduce the speed, while a medium probability of a corner impact might trigger warning to steer away from the obstacles. The actual mapping defined for this project will be much more complex, since the output from the sensor is far more detailed than these examples imply. 


\section{SENSOR TESTING AND MODELING}

This section summarizes the tasks completed during Sensor Testing and Modeling. These include:

- Literature Review

- $\quad$ Refine Test Matrix

- $\quad$ Sensor Modeling

- Sensor Baseline Testing

- Sensor Simulations

Several of the tasks deal with investigation of specific sensors such as a magnetic sensor, radar sensor and ultrasonic sensor. The same sensors appear in multiple tasks. It was decided to organize this section by task instead of by sensor in order to be consistent with the project proposal and organization.

\subsection{LITERATURE REVIEW}

Two desired outcomes from the literature review were to:

- Collect accident data and identify a project focus area.

- Identify state-of-the art sensors and their capabilities and limitations.

\subsubsection{Accident Data}

Truck accident data was reviewed to confirm the type and circumstances of current accidents. From this data, and information about the current commercial activities relating to accident prevention, a research focus area was identified.

The Federal Motor Carrier Safety Administration (FMCSA) Large Truck Crash Causation Study (LTCCS) [1] reports that rear-end collisions represent $30 \%$ of class 8 truck accidents. Half of those rear-end collisions (14\% of all class 8 accidents) involve an object impacting the rear of the truck trailer. No existing or proposed commercial warning system is focused on this area. 
Sideswipe collisions represent $12 \%$ of class 8 truck accidents. Half of these ( $6 \%$ of all class 8 accidents) occur when another vehicle encroaches on the truck's lane, while $40 \%$ (5\% of all accidents) involve the truck encroaching on another vehicle. Many side-detection systems are in production or under development to warn the truck driver of objects nearby, but most act only if a lane change is anticipated. Current and proposed commercial side detection systems do not warn other vehicles or interact with rear-facing sensors to anticipate problems.

Based on this data, the team chose to focus on a sensing system to prevent rear-impact and sideswipe crashes. Both long- and short-range sensor were considered for these crash modes, as described in the next section.

\subsubsection{State-of-the-art Sensors}

A thorough literature review was completed to identify the state-of-the-art sensors and their applications in today's crash avoidance systems. Most technical research in this area focuses on a specific type of sensor and its application limitations. Instead of focusing on a particular sensor type, this literature search was aimed at reviewing all sensor types being used in crash avoidance and similar applications.

Although many different crash avoidance systems were identified, all of them rely on one of more of the following technologies: RADAR, LIDAR, computer vision, ultrasonic, infrared, or magnetoresistive.

\section{RADAR}

Radar is a well-known and commonly used sensor in today's crash avoidance systems. The one factor that separates Radar from other sensors is its ability to operate in adverse weather conditions such as rain, fog, and dirty environments. This sensor is also of interest for its market 
availability and its ability to measure range, relative velocity, and angle, as well as detect and track multiple targets.

Radar is a key technology for automotive applications of driver assistance and safety. Compared to other technologies used for the same purpose it is a robust and proven technology. In addition radar operates under bad weather conditions, which is usually the most critical time a driver will need assistance [2].

Currently cars equipped with $24 \mathrm{GHz}$ Short Range Radar (SSR) systems in combination with 77 GHz Long Range Radar (LRR) are already in the market [3]. The used of the LRR has been mostly for adaptive cruise control [4]. Possible applications for SRR include collision warning and mitigation, blind spot monitoring, and lane change assistance [2]. Recently, a number of automotive suppliers have developed proprietary $24 \mathrm{GHz}$ SRR concepts [5].

\section{LIDAR}

LIDAR measures distance and speed by analyzing the time of flight and Doppler shift of its transmitted light signal. The source of light is usually transmitted from a set of LEDs with a narrow field of view. The light signals can also be transmitted in an array or progressively scanned to achieve a wider field of view. LIDAR is able to measure distance and speed accurately, but this accuracy can decrease if the laser's optics is affected by dirt or rain. The literature search indicated LIDAR performance can be similar to RADAR, but at a reduced price [6].

\section{Computer Vision}

Computer vision consists of one or more cameras to characterize a vehicle's environment or monitor driver state. Computer vision can be found in blind spot detection, parking aid, and driver fatigue warning systems [6]. These systems work by sending digital pictures to a processor that 
converts these images into data such as distance, speed, and image identification. Using computer vision requires a great deal of processing, but the possibilities for a crash avoidance system are only limited by the processor speed and picture resolution. When using this type of sensor, processing speed is of large concern because the time it takes to process an image may delay a warning to the driver. Weather also can affect the processing of this system. Fog, dirt, and the sun may affect the image quality and thus the output data of to the system.

\section{Ultrasonic Sensor}

The use of these sensors in active crash avoidance applications is not common due to the adverse environment the sensor must endure, including weather and high vehicle speeds. Our review focused on finding applications of these sensors in a trucking environment. Several studies have investigated ultrasonic sensor performance during highway driving [7], [8]. These reports claim that wind turbulence causes errors in the sensor measurements. It was decided that the low-cost and range measurement benefits of these sensors was worth corroborating these claims.

\section{Infrared Sensor}

Infrared sensors can be classified into two categories: active and passive. These sensors have the ability to measure distance and speed by measuring the change in intensity of the reflected infrared beams. An active infrared sensor emits a scatter of infrared pulses and detects the reflected signals. The passive infrared sensor measures incoming infrared waves emitted by a heat source without transmitting any signal. In crash avoidance systems, these heat sources may be pedestrian, animals, or even engine heat. Use of infrared sensors in crash avoidance applications is limited because they are affected by sun, headlights, and other heat sources [6]. 


\section{Magneto-resistive Sensor}

The inherent ability of magnetic sensors to accurately detect the changes in the earth's magnetic field has resulted in their primary use as navigation devices for over 2,000 years. In the more recent past, extensive research has resulted in the invention of different types of magnetic sensors that are capable of measuring magnetic field strengths from $\mu$ Gauss to MGauss [9]. These magnetic sensors are capable of directly measuring the earth's magnetic field as well as localized changes in this magnetic field (presence of ferromagnetic materials). Typical magneto-resistive sensors are low cost, high sensitivity magnetic devices with a measurement range from several $\mu$ Gauss to tens of Gauss [10]. In addition, their small size and resilience to harsh environments have led to their extensive use in varied applications such as navigational systems [11], [12], traffic surveillance [13], [14], and vehicle detection [12], [15], [16]. Some research has also been conducted on the use of magnetic sensors onboard vehicles for proximity and blind spot detection [17], [18]. Based on this background information, a similar magneto-resistive type sensor has been utilized in this project for the purpose of vehicle type detection.

\subsection{ADDITIONAL SENSORS}

This section consists of the consideration and procurement of sensors for inclusion in the project, based on the results of the literature search. Potential sensor candidates were evaluated based on their cost, signal characteristics and availability. Sensors procured include:

- Radar sensor - $24 \mathrm{GHz}$ RADAR sensors were donated by an OEM sensor manufacturer.

- Computer Vision sensor - A Uni-brain fire wire camera was purchased to act as a test validation tool as well as being used for image processing. The camera resolution is $640 \times 480$ with a FOV of $42^{\circ}$ horizontally and $32^{\circ}$ vertically [19].

- Ultrasonic sensor - MaxBotix $42 \mathrm{KHz}$ LV-MaxSonar®-EZ1 ultrasonic sensors were purchased [20]. This sensor transmits a pulsed inaudible $42 \mathrm{kHz}$ sound wave at a sampling rate of $20 \mathrm{~Hz}$ to detect range up to $6 \mathrm{~m}$. Range is measured 
using a time-of-flight (TOF) method, where the time it takes for a transmitted signal to return to the sensor face is analyzed. Relating this time and the velocity of sound the range can be determined.

- Magneto-resistive sensor - The particular sensor selected for the present study is the HMC 2003 series 3-axis magneto-resistive sensor manufactured by Honeywell [10]. This sensor type has been shown to function as either a compass by measuring the earth's magnetic field with respect to the sensor's orientation or as a vehicle detecting device by measuring only localized distortions in a magnetic field (presence of ferromagnetic material) [12]. This study focuses solely on vehicle detection and any effect due to the earth's constant magnetic field and sensor orientation is filtered out for all the analyses presented. This sensor uses three nickel-iron, permalloy magneto-resistive sensors with a magnetic field sensing range of 2 Gauss and has a resolution of $40 \mu$ Gauss. With a sensing bandwidth of $1 \mathrm{kHz}$, this sensor is capable of vehicle proximity detection even at high relative speeds [10].

Based on the literature search, each of these sensors appeared to meet the project goals of low cost, readily available, and having useful signal characteristics. Two other possible sensors were identified during the literature review but were later rejected: A scanning LIDAR sensor could not obtained at a reasonable cost for the project. Infrared (IR) sensors were rejected due to their inherent limitations (confusion by existing IR sources) and development cost (image processing).

Each of the procured sensors were tested, modeled, and evaluated for their potential to contribute to the goals of the project. These steps will be discussed for each sensor in the following sections.

\subsection{REFINE TEST MATRIX}

The original project anticipated the investigation of radar and ultrasonic sensors and included testing for these sensors. As discussed, magneto-resistive and computer vision sensors were also selected as possible sensor candidates. This section revises the testing matrix to include the magneto-resistive and computer vision sensors. 
The magneto-resistive sensor has not been used for this application in the past and its characteristics are not well established. Therefore testing was planned that would characterize the sensor behavior under a tightly controlled environment. This consisted of a special test fixture on a lab bench setting. Next static and mobile traffic environment testing was planned. The results of these tests are described along with the other sensors in Task 6 - Baseline Testing.

The computer vision sensor also required testing to verify its capabilities. A basic test plan was developed to record video in relevant settings (on-highway, multiple vehicles, daylight) and successively test the computer vision algorithms with one, two, and several vehicles. Since the major effort for vision sensing is in the processing algorithms, this was the extent of the baseline test plan. If algorithm development proceeds smoothly, additional video will be collected in different conditions (hill climb, nighttime, fog, etc).

\subsection{SENSOR MODELING}

Mathematical models of the sensors were constructed to provide a means of simulating the performance of the sensors and to facilitate algorithm development of the project. In addition, the modeling helped to understand the behavior of the sensors when it was not obvious (especially with the magnetic sensor).

\subsubsection{Magnetic Sensor}

The Honeywell HMC 2003 series three-axis anisotropic magnetic sensor hybrid has its sensor elements oriented as a resistive "Wheatstone bridge" that varies resistance slightly as the magnetic field changes in each element. This change in resistance causes a change in output voltage, whose voltage magnitude is related to the induced magnetic field by the sensitivity equation (1).

$$
(\text { Out }+) \text { - (Out- })=\mathrm{S}^{*} \mathrm{~V}_{\mathrm{b}} * \mathrm{~B}_{\mathrm{s}}
$$


where,

$$
\begin{aligned}
& \mathrm{S}=\text { Sensitivity (nominally } 1 \mathrm{mV} / \mathrm{V} / \text { Gauss), } \\
& \mathrm{V}_{\mathrm{b}}=\text { Bridge supply voltage (Volts), and } \\
& \mathrm{B}_{\mathrm{s}}=\text { Bridge applied magnetic flux (Gauss). }
\end{aligned}
$$

The values of $S$ and $V_{b}$ in the above expression are constants and depend only on the sensor type. However, the magnitude of $B_{s}$ depends on the properties of the sensed object, which, in our case are magnets used in laboratory tests and vehicles in field testing. Hence, it was necessary to model the magnetic source to produce the induced field strength $B_{s}$.

\section{Simple 2-D Dipole Modeling}

In literature, a 2-D dipole modeling approach was undertaken to capture the magnetic behavior of vehicles [14], [15]. In these studies, it was mentioned that each of the automobile axles behaved as a magnet and it was concluded that the total induced field of the vehicle could be adequately represented by a single magnet (single dipole) [14]. To ascertain the validity of these claims and conclusions, a simple 2-D single dipole model similar to the one proposed in [14] was developed and is described below.

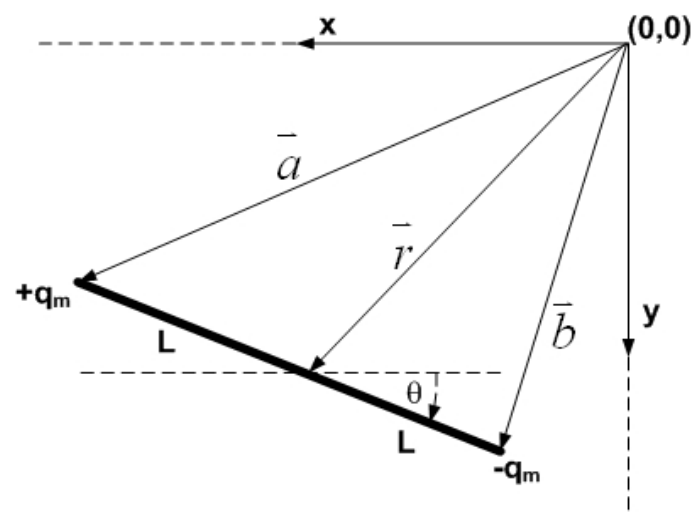

Figure 1. 2-D single magnetic dipole model.

Consider the 2-D dipole model shown in Fig. 1. The dipole in this model corresponds to the axially magnetized permanent magnet that was used in the bench test. The origin of the co- 
ordinate axes in the figure is where the magnetic sensor is considered to be located. For the purposes of model development, assume that the dipole is comprised of two equal but opposite magnetic charges $+q_{m}$ and $-q_{m}$ separated by a distance $2 L$. The center of the dipole is considered to be located at coordinates $(x, y)$ with respect to the origin and makes an angle of $\theta$ with the $\mathrm{x}$ axis.

For this particular configuration, the magnetic field $B_{s}$ induced by the dipole at the sensor location is given by the well-known expression,

$$
\overrightarrow{B_{s}}=\frac{\mu q_{m}}{4 \pi}\left(\frac{\vec{a}}{a^{3}}-\frac{\vec{b}}{b^{3}}\right)
$$

where,

$$
\begin{aligned}
& \mu=\text { magnetic permeability of the magnet material, and } \\
& q_{m}=\text { magnetic charge strength of the dipole. }
\end{aligned}
$$

From vector algebra we have,

$$
\begin{aligned}
& \vec{a}=\vec{r}+\vec{u} L \\
& \vec{b}=\vec{r}-\vec{u} L
\end{aligned}
$$

where,

$\vec{u}$ - unit vector along the dipole length.

$\vec{r}$ - distance vector from the dipole center to the origin.

$L$ - half length of dipole.

The unit vector $\vec{u}$ and $\vec{r}$ are given by

$$
\begin{gathered}
\vec{u}=\cos (\theta) \vec{\imath}-\sin (\theta) \vec{\jmath} \\
\vec{r}=x \vec{\imath}+y \vec{\jmath}
\end{gathered}
$$

Substituting (5) and (6) into (3) and (4) we get;

$$
\begin{aligned}
& \vec{a}=(x+L \cos (\theta)) \vec{\imath}+(y-L \sin (\theta)) \vec{\jmath} \\
& \vec{b}=(x-L \cos (\theta)) \vec{\imath}+(y+L \sin (\theta)) \vec{\jmath}
\end{aligned}
$$

Finally, substituting (7) and (8) into (2) and simplifying we get; 


$$
\overrightarrow{B_{s}}=\left(\frac{\mu q_{m}}{4 \pi}\right)\left[\frac{x+L \cos (\theta)}{a^{3}}-\frac{x-L \cos (\theta)}{b^{3}}\right] \vec{\imath}+\left(\frac{\mu q_{m}}{4 \pi}\right)\left[\frac{y-L \sin (\theta)}{a^{3}}-\frac{y+L \sin (\theta)}{b^{3}}\right] \vec{\jmath}
$$

The above expression is of the form

$$
\overrightarrow{B_{s}}=B_{x} \vec{\imath}+B_{y} \vec{\jmath}
$$

In (2), the terms $\mu$ and $q_{m}$ are theoretical quantities that are difficult to ascertain in real-world applications and have been replaced by empirical relations as shown below.

$$
\begin{aligned}
& B_{x}=K_{x}\left[\frac{x+L \cos (\theta)}{a^{3}}-\frac{x-L \cos (\theta)}{b^{3}}\right] \\
& B_{y}=K_{y}\left[\frac{y-L \sin (\theta)}{a^{3}}-\frac{y+L \sin (\theta)}{b^{3}}\right]
\end{aligned}
$$

The predictions of this model will be compared against the bench test results later in this report.

\section{3-D Mathematical Model Development}

The road tests mentioned above involved the measurement of vehicle magnetic footprints along $x$, $y$, and $z$ axes. Compared to the 2-D bench tests, the $z$ axis was also considered for measurement increasing the model dimension to 3-D. Although, the added dimension increases the complexity of the model, it provides additional information on the magnetic footprints that could be utilized to better distinguish the different vehicle types.

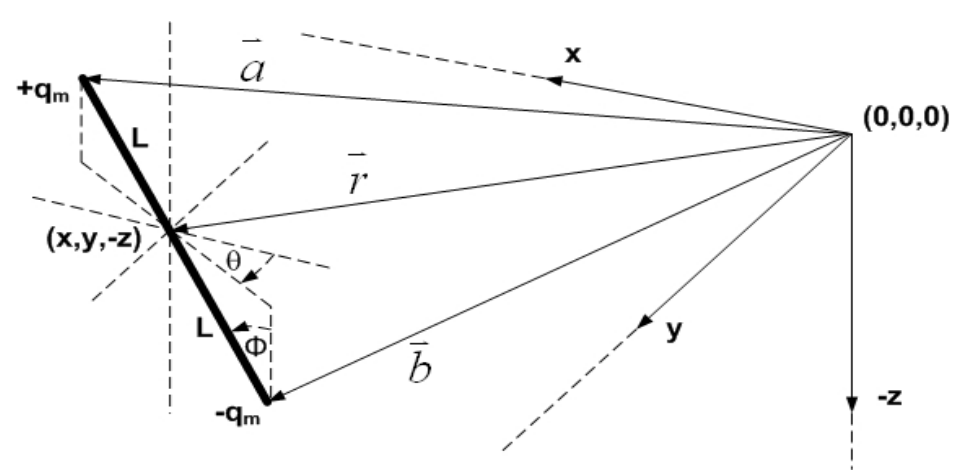

Figure 2. Magnetic dipole in 3-D space.

Consider a dipole of length $2 L$ and having magnetic charge strength of $q_{m}$ similar to that shown in

Fig. 1. However, the dipole is now oriented in 3-D space with its center located at coordinates $(x$, 
$y$, and $-z$ ) as shown in Fig. 2. Equations (3) and (4) still hold true for the three dimensional case; however, the vectors $\vec{u}$ and $\vec{r}$ are now three dimensional and given by the expressions (13) and (14).

$$
\begin{gathered}
\vec{u}=\cos (\theta) \sin (\phi) \vec{\imath}-\sin (\theta) \sin (\phi) \vec{\jmath}-\cos (\phi) \vec{k} \\
\vec{r}=x \vec{\imath}+y \vec{\jmath}-z \vec{k}
\end{gathered}
$$

Substituting (13) and (14) into (3) and (4) we get;

$$
\begin{aligned}
& \vec{a}=(x+L \cos (\theta) \sin (\phi)) \vec{\imath}+(y-L \sin (\theta) \sin (\phi)) \vec{\jmath}+(-z+L \cos (\phi)) \vec{k} \\
& \vec{b}=(x-L \cos (\theta) \sin (\phi)) \vec{\imath}+(y+L \sin (\theta) \sin (\phi)) \vec{\jmath}+(-z-L \cos (\phi)) \vec{k}
\end{aligned}
$$

Further, substituting (15) and (16) into (2) and simplifying we get;

$$
\begin{aligned}
& \overrightarrow{B_{s}}=\left(\frac{\mu q_{m}}{4 \pi}\right)\left[\frac{x+L \cos (\theta) \sin (\phi)}{a^{3}}-\frac{x-L \cos (\theta) \sin (\phi)}{b^{3}}\right] \vec{\imath} \\
& +\left(\frac{\mu q_{m}}{4 \pi}\right)\left[\frac{y-L \sin (\theta) \sin (\phi)}{a^{3}}-\frac{y+L \sin (\theta) \sin (\phi)}{b^{3}}\right] \vec{\jmath} \\
& +\left(\frac{\mu q_{m}}{4 \pi}\right)\left[\frac{-z+L \cos (\phi)}{a^{3}}-\frac{-z-L \cos (\phi)}{b^{3}}\right] \vec{k}
\end{aligned}
$$

where,

$$
\begin{aligned}
& a=\sqrt{(x+L \cos (\theta) \sin (\phi))^{2}+(y-L \sin (\theta) \sin (\phi))^{2}+(-z+L \cos (\phi))^{2}} \\
& b=\sqrt{(x-L \cos (\theta) \sin (\phi))^{2}+(y+L \sin (\theta) \sin (\phi))^{2}+(-z-L \cos (\phi))^{2}}
\end{aligned}
$$

Equation (17) is of the form:

$$
\overrightarrow{B_{s}}=B_{x} \vec{\imath}+B_{y} \vec{\jmath}+B_{z} \vec{k}
$$

Similar to (9) the terms $\mu$ and $q_{m}$ in (17) are theoretical quantities that are difficult to ascertain and are replaced by empirical co-relations (21)-(23).

$$
B_{x}=K_{x}\left[\frac{x+L \cos (\theta) \sin (\phi)}{a^{3}}-\frac{x-L \cos (\theta) \sin (\phi)}{b^{3}}\right]
$$




$$
\begin{gathered}
B_{y}=K_{y}\left[\frac{y-L \sin (\theta) \sin (\phi)}{a^{3}}-\frac{y+L \sin (\theta) \sin (\phi)}{b^{3}}\right] \\
B_{z}=K_{z}\left[\frac{-z+L \cos (\phi)}{a^{3}}-\frac{-z-L \cos (\phi)}{b^{3}}\right]
\end{gathered}
$$

Equations (21)- (23) provide the expressions relating the magnetic fields along the $x, y$, and $z$ axes to the model parameters; dipole length $(L)$, dipole angles $(\theta, \varphi)$, location of dipole center $(x, y, z)$, and the empirical co-relations $\left(K_{x}, K_{y}, K_{z}\right)$. To better understand the effect that each of these model parameters has on the field strengths, a detailed parameter simulation study was carried out.

\section{Parameter Simulation Study}

The behavior of the magnetic field induced by a dipole is dependent on its geometry and spacial orientation with respect to the sensor. Hence, a detailed parameter study is required to isolate the effects of each of these parameters on the model response. As mentioned above, the different model parameters are; dipole length $(L)$, dipole angles $(\theta, \varphi)$, location of dipole center $(x, y, z)$ and the empirical co-relations $\left(K_{x}, K_{y}, K_{z}\right)$. From (21)-(23), it can be noticed that the empirical corelations $\left(K_{x}, K_{y}, K_{z}\right)$ are simple multiplying factors and hence have only a scaling effect on the magnitudes of the magnetic fields. Also, since the dipole is considered to move along the $y$-axis of the sensor, the effects of the dipole y coordinate is implicit in all the parameter study plots. The following paragraphs describe the effects of each of the other parameters individually. 

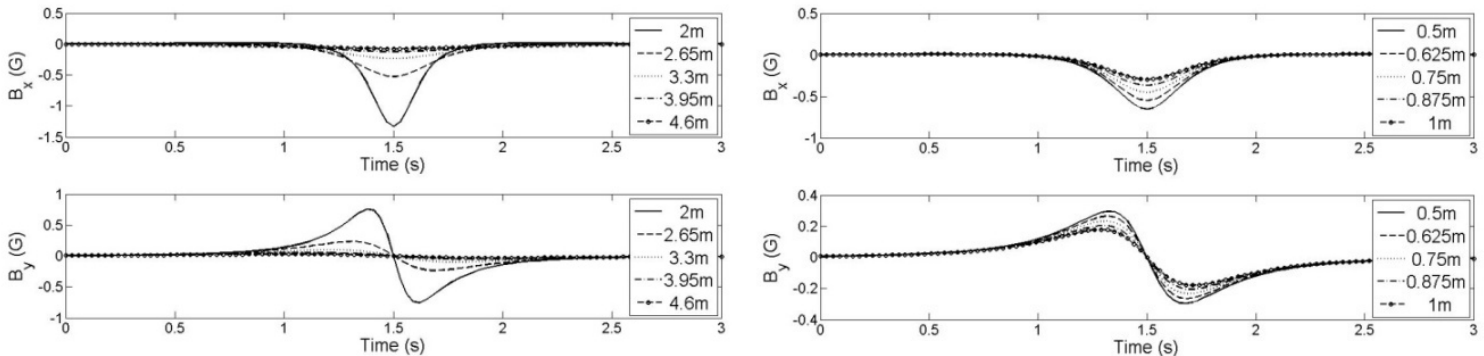

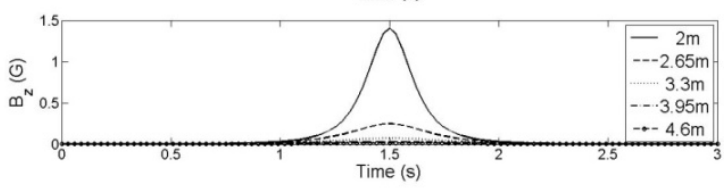

(a) Dipole X-coordinate location

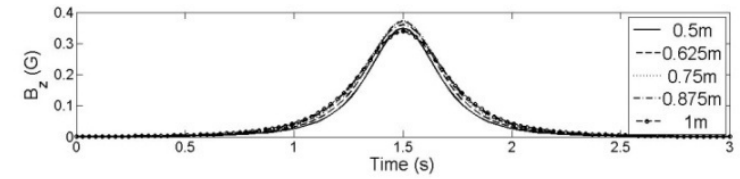

(b) Dipole z-coordinate location

Figure 3. Effect of dipole location

\section{Dipole Coordinate Location}

From Fig. 3, it can be seen that the $x$ coordinate location of the dipole decides the lateral position of the dipole with respect to the sensor location. Simulations were carried out by varying this distance from $2 \mathrm{~m}$ to $4.6 \mathrm{~m}$ and the results are as shown in Fig. 3. As expected, the magnitude of the magnetic footprints in all the axes increase as the $x$ distance is reduced.

The effect of the $z$ coordinate location of the dipole is more interesting (Fig. 3). While the magnitudes of the induced magnetic fields along the $x$ and $y$ axes peak when $z=0$, the behavior observed along the $z$ measurement axis shows a different trend. From Fig. 3, it can be observed that as the $z$ value is decreased, starting from a value of zero, the magnitude of $B_{z}$ approaches a maximum at $z=-1$ before decreasing and eventually tending to zero as the $z$ coordinate further decreases. Similar trend will be observed from symmetry for positive values of $z$. 

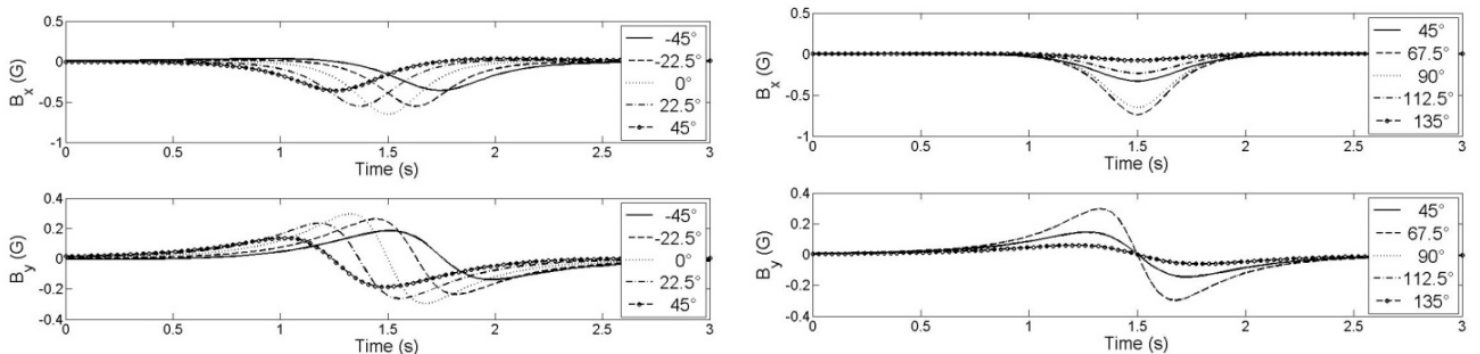

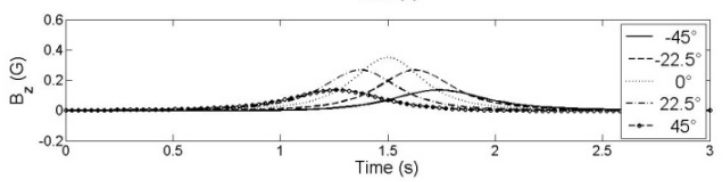

(a) Dipole bearing $(\theta)$.

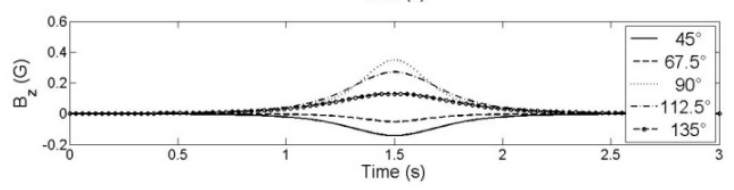

(b) Dipole bearing $(\varphi)$.

Figure 4. Effects of dipole angle.

\section{Dipole Bearing}

Again from Fig. 2, the dipole was assumed to make an angle $\theta$ with the $x$-axis and an angle $\varphi$ with the $z$-axis. The angle $\theta$ orients the dipole in one of two positions depending on whether $\theta$ is positive or negative. In the case when $\theta$ is positive, the magnetic sensor sees the magnetic pole closest to the sensor quicker than if the angle $\theta$ were negative. Hence, as $\theta$ is changed from its positive maximum to its negative minimum, the effect is that of a delayed sensing on the part of the sensor (Fig. 4). This delay appears to be symmetric on either side of the zero bearing. Also, it can be observed that changing $\theta$ has little effect on the magnetic field strengths $B_{x}$ and $B_{y}$, but alters the magnitude of $B_{z}$ more considerably.

Figure 4 also shows the effect of changing the dipole angle $\varphi$, which is the angle that the dipole was assumed to make with the $z$-axis (Fig. 2). As compared to the effect of changing the angle $\theta$, where the magnetic trend was continuous and changed monotonically, the angle $\varphi$ has an effect similar to that of changing the $z$ coordinate location. It can also be noticed from Fig. 4 that the magnitudes of $B_{x}, B_{y}$, and $B_{z}$ are the largest when the dipole is oriented in the $x-y$ plane $\left(\varphi=90^{\circ}\right)$.

The effect of changing the dipole length is shown in Fig. 5. It can be observed that increasing the length of the dipole has a similar effect to increasing the dipole magnetic intensity. This is 
because, increasing the length increases the distance of the $+q_{m}$ magnetic pole from the sensor location causing the $-q_{m}$ magnetic pole to produce an enhanced effect at the same location without cancellation (Fig. 2).
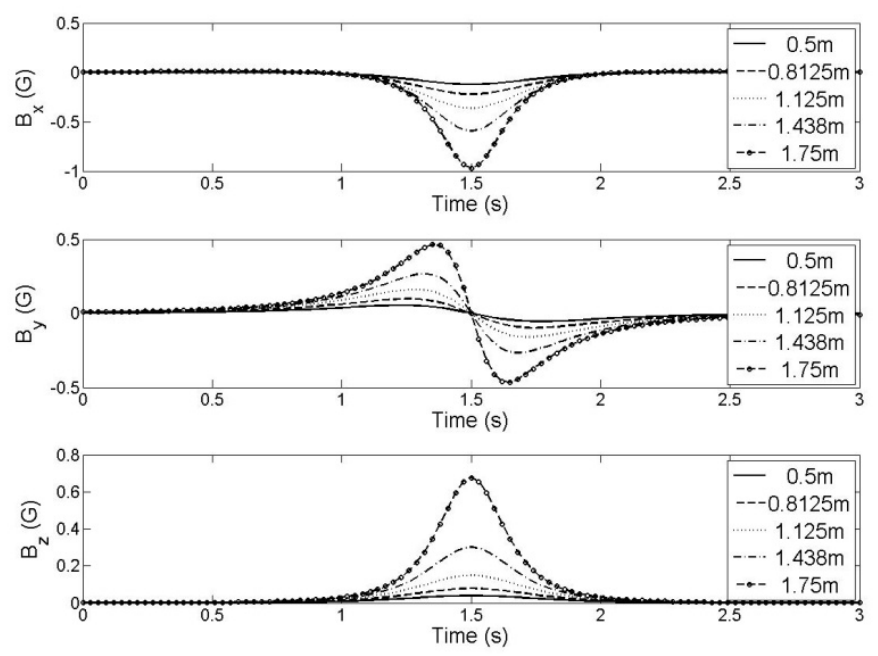

Figure 5. Effects of dipole length $(L)$.

Equations (21)-(23) provided magnetic field strength information for a single dipole along the $x$, $y$, and $z$ axes. However, the magnetic profiles of vehicles obtained from road tests showed higher order dynamics suggesting the presence of multiple dipoles (Fig. 7). This complex behavior was assumed to be due to superposition effects and could be captured by modeling these vehicles as multiple dipoles (Fig. 6). A slight modification to the single dipole model (21)-(23) yields an $n$ dipole model that was used to model cars and trucks (24)-(26).

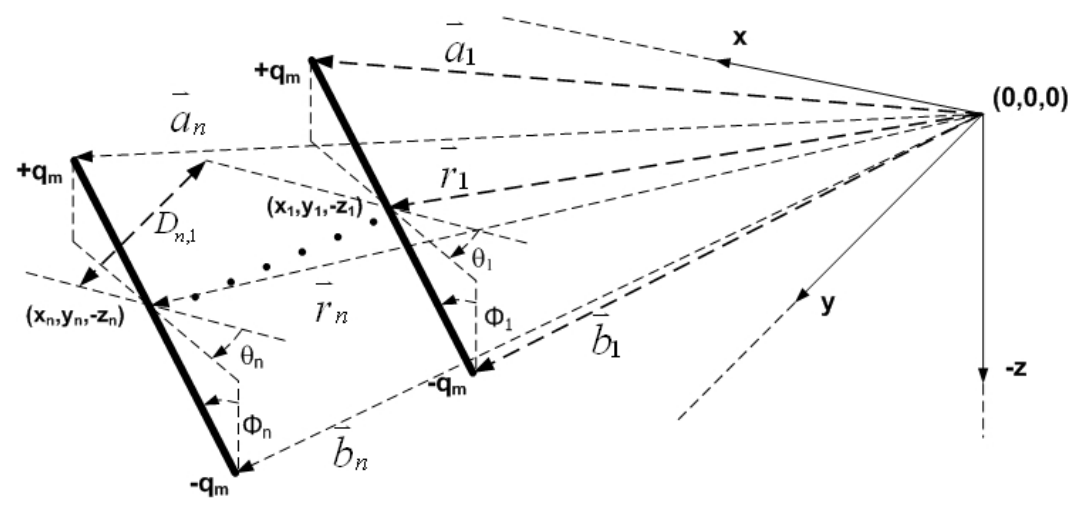

Figure 6. Multi-dipole model schematic. 


$$
\begin{gathered}
B_{x}=\sum_{n=1}^{n} K_{x_{n}}\left[\frac{x_{n}+L_{n} \cos \left(\theta_{n}\right) \sin \left(\phi_{n}\right)}{a_{n}^{3}}-\frac{x_{n}-L_{n} \cos \left(\theta_{n}\right) \sin \left(\phi_{n}\right)}{b_{n}^{3}}\right] \\
B_{y}=\sum_{n=1}^{n} K_{y_{n}}\left[\frac{y_{1}+D_{n, 1}-L_{n} \sin \left(\theta_{n}\right) \sin \left(\phi_{n}\right)}{a_{n}^{3}}-\frac{y_{1}+D_{n, 1}-L_{n} \cos \left(\theta_{n}\right) \sin \left(\phi_{n}\right)}{b_{n}^{3}}\right] \\
B_{z}=\sum_{n=1}^{n} K_{z_{n}}\left[\frac{-z_{n}+L_{n} \cos \left(\phi_{n}\right)}{a_{n}^{3}}-\frac{-z_{n}-L_{n} \cos \left(\phi_{n}\right)}{b_{n}^{3}}\right]
\end{gathered}
$$

where,

$$
D_{n, 1} \text { - distance between the } 1^{\text {st }} \text { dipole and the } \mathrm{n}^{\text {th }} \text { dipole measured along the } \mathrm{y} \text {-axis }
$$

$$
\begin{aligned}
& a_{n} \\
& =\sqrt{\left(x_{n}+L_{n} \cos \left(\theta_{n}\right) \sin \left(\phi_{n}\right)\right)^{2}+\left(y_{1}+D_{n, 1}-L_{n} \sin \left(\theta_{n}\right) \sin \left(\phi_{n}\right)\right)^{2}+\left(-z_{n}+L_{n} \cos \left(\phi_{n}\right)\right)^{2}} \\
& b_{n} \\
& =\sqrt{\left(x_{n}-L_{n} \cos \left(\theta_{n}\right) \sin \left(\phi_{n}\right)\right)^{2}+\left(y_{1}+D_{n, 1}-L_{n} \cos \left(\theta_{n}\right) \sin \left(\phi_{n}\right)\right)^{2}+\left(-z_{n}-L_{n} \cos \left(\phi_{n}\right)\right)^{2}}
\end{aligned}
$$

From the insights gained in the parameter simulation study, this multi-dipole model was tuned to obtain a good representation for the magnetic behavior of a typical passenger car and a truck. The 3-D mathematical model comprised of 2 dipoles for modeling the magnetic effects of cars and 5 dipoles to get a reasonably accurate representation of typical class-8 trucks. As shown in Fig. 7, the dipole locations for both cars and trucks closely relate to the metal concentration areas such as wheel axles, engine manifold, etc where the magnetic field lines get concentrated.

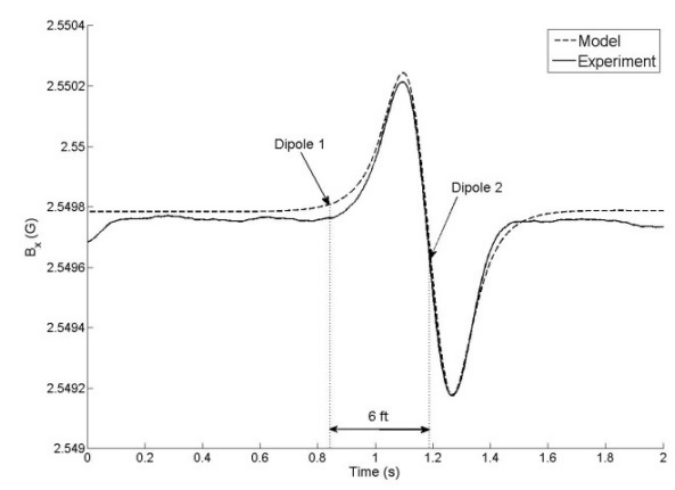

(a) Passenger car.

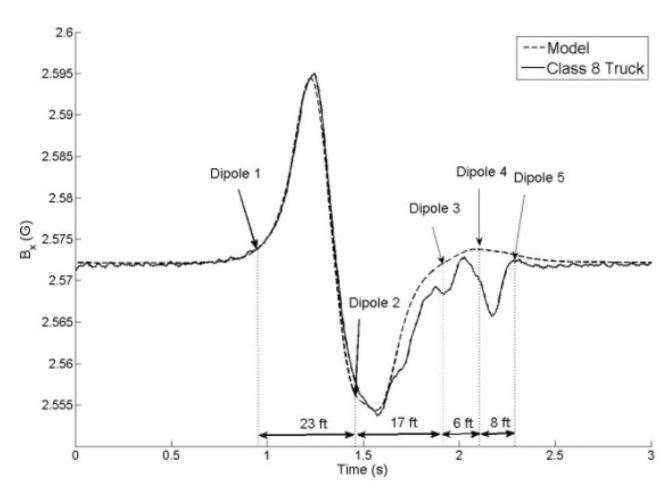

(b) Class 8 truck.

Figure 7. Dipole locations for vehicles. 


\subsubsection{Ultrasonic Sensor}

The MaxBotix $42 \mathrm{KHz}$ LV-MaxSonar®-EZ1 is a piezoelectric transducer that emits an inaudible sound wave when electricity is applied to it. The time it takes to receive an echo from a transmitted signal can be analyzed to measure range. Although ultrasound sensors are well understood for low-speed vehicle applications, the added complexities of operating at highway speeds and the increased reliability for a safety (versus convenience) application necessitated further study. Modeling focused on how these other factors influence the detection zone and predicted distances.

\section{Ultrasonic Sensor Environmental Effects}

In a laboratory setting ultrasonic sensors have a simple linear relationship between range and sensor output voltage; however, the adverse environmental elements that this sensor will endure in this crash avoidance application may affect this simple relationship. This model may be modified to include effects of relative humidity, air temperature, vehicle speed, and wind turbulence. Each of these effects influences the velocity of sound and thus, influences the range measurement of the ultrasonic sensor.

From our literature review, it has been shown that changes in the relative humidity may be neglected because the change in the velocity of sound is relatively small. For example, the change in the velocity of sound is only $0.15 \%$ over a range of $10 \% \mathrm{RH}-90 \% \mathrm{RH}$ at $20^{\circ} \mathrm{C}$ [7].Effects from temperature may also be neglected for this specific application. It has been reported that the effect of temperature on the velocity of sound $[\mathrm{m} / \mathrm{s}]$ in air may be approximated with the function:

$$
V_{s} \approx 20.055 \sqrt{T}
$$

where $T$ is absolute temperature in Kelvin [7]. Class 8 vehicle operating temperature range may be assumed to be $-30^{\circ} \mathrm{C}$ to $+55^{\circ} \mathrm{C}$. In this range, this ultrasonic sensor may have up to $\sim 8 \%$ uncertainty in its range measurement. Because this sensor's primary function is to detect the 
presence of a vehicle in the adjacent lane and precise distance measurement is of secondary importance, this error may be neglected.

Uncertainty of an ultrasonic sensor due to vehicle speed $V_{w}$, which has the same effect of the component of the wind that flows along the length of the vehicle, has been reported to be [7]:

$$
\frac{\Delta D}{D} \approx \frac{1}{2} \cdot\left(\frac{V_{w}}{V_{s}}\right)^{2}
$$

Again, this error is neglected because the error of a vehicle at highway speeds at $20^{\circ} \mathrm{C}$ is only $\sim 0.35 \%$.

Mathematical modeling of air turbulence over the ultrasonic sensor was considered; however, due to the nontrivial approach of modeling air turbulence in various conditions, an experimental approach to reduce turbulence was taken. The approach taken to reduce air turbulence was simple shielding. This will be discussed in the testing section.

In the end, although temperature and vehicle speed can be considered in the ultrasonic sensor algorithm, their effect is likely insignificant for the purposes of this study.

\section{Ultrasonic Sensor Sample Rate}

Most current ultrasonic sensors are used in low speed scenarios such as parking aids; however, in a crash avoidance system the sensor must operate properly over a range of vehicle speeds. For a side-mounted ultrasonic sensor, at higher vehicle speeds it is possible to pass an object without detecting it if the sensor sampling rate is too slow. In order to verify the effectiveness of a sidemounted ultrasonic sensor the relation between sensor sample rate, vehicle speed, and the minimum detectable length were studied. This relationship can be represented as $L=v / f$, where $\mathrm{L}$ is the minimum detectable object length, $\mathrm{f}$ is the sensor sample rate in $\mathrm{Hz}$, and $\mathrm{v}$ is the relative speed. Figure 8 shows this relation for ultrasonic sensors with various sample rates. The sensor 
procured for this study operates at a sample rate of $20 \mathrm{~Hz}$ and therefore will receive a return signal for objects $1.4 \mathrm{~m}$ or longer at $100 \mathrm{~km} / \mathrm{hr}$; this is the minimum distance required to measure at least one data point. In the worst case scenario where the host vehicle passes a stopped motorcycle ( $2 \mathrm{~m}$ long) on the side of the highway at $100 \mathrm{~km} / \mathrm{hr}$, the selected ultrasonic sensor will be able to detect this object.

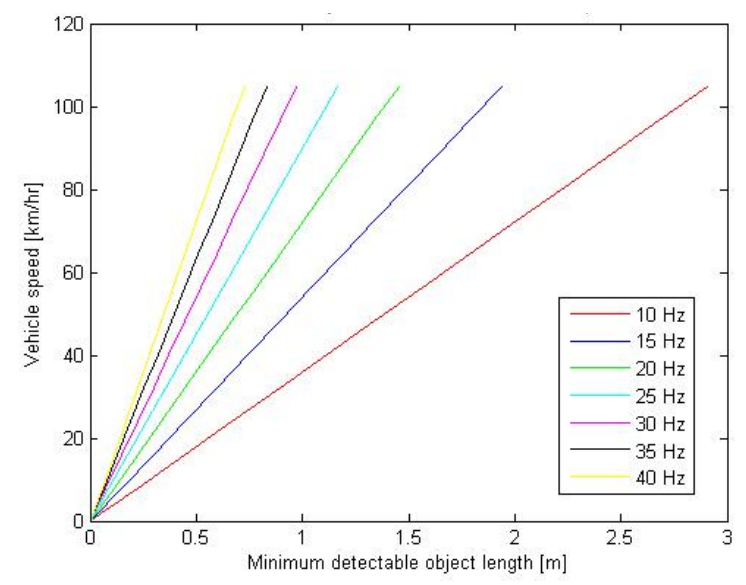

Figure 8. Minimum object sizes for side-mounted ultrasonic sensor return signal.

\section{Ultrasonic Sensor Placement}

Placement of the ultrasonic sensors is crucial because a set of sensors must monitor an area that covers the entire length of a class 8 truck's trailer. To ensure that a vehicle can be detected along the entire length of the trailer the ultrasonic sensors may be placed at an angle $\theta$ relative to the trailer of the large truck (Fig. 9). This allows a single ultrasonic sensor to be used to monitor a region rather than detect what is directly adjacent to the trailer. The ultrasonic sensor spacing and angle settings must be selected to ensure that the crash avoidance system is aware of a vehicle's presence as the vehicle moves along the length of the trailer and as it transitions from one ultrasonic sensing region to the subsequent one. With the assumption that the ultrasonic sensors are mounted at a fixed angle and only detect targets on their centerline, a geometric relationship has been defined between object length, road parameters, sensor capabilities, and sensor spacing. Figure 9 shows a class 8 truck trailer with two ultrasound sensors mounted on its lateral side and 
a motorcycle (minimum detectable threat) in the adjacent lane. Table 1 shows equations and values used to determine the minimum separation. The minimum separation between two ultrasonic sensors is the sum of the target length and a component of the target width.

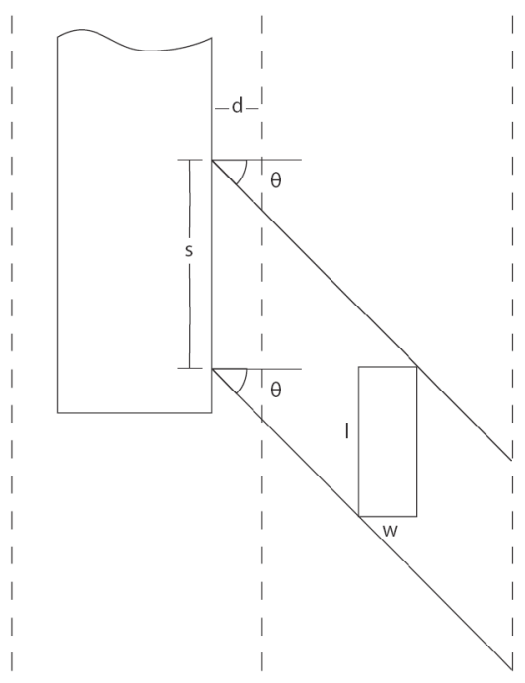

Figure 9. Coverage zone for side-mounted ultrasonic sensors.

TABLE 1. Variables and equations to determine ultrasonic sensor placement

\begin{tabular}{|c|c|c|c|}
\hline Variable & Description & Equation & Motorcycle \\
\hline $\mathrm{r}$ & Ultrasonic max range & $\mathrm{n} / \mathrm{a}$ & $6.0 \mathrm{~m}$ \\
\hline $\mathrm{w}$ & Lane width & $\mathrm{n} / \mathrm{a}$ & $3.5 \mathrm{~m}$ \\
\hline $\mathrm{wt}$ & Trailer width & $\mathrm{n} / \mathrm{a}$ & $2.5 \mathrm{~m}$ \\
\hline $\mathrm{wm}$ & Motorcycle width & $\mathrm{n} / \mathrm{a}$ & $1.0 \mathrm{~m}$ \\
\hline $\mathrm{lm}$ & Motorcycle length & $\mathrm{n} / \mathrm{a}$ & $2.0 \mathrm{~m}$ \\
\hline $\mathrm{d}$ & Distance to lane divider & $d=\frac{w-w_{t}}{2}$ & $0.5 \mathrm{~m}$ \\
\hline$\theta$ & Ultrasonic sensor angle & $\theta=\cos ^{-1}\left(\frac{d+w}{r}\right)$ & $48^{\circ}$ \\
\hline $\mathrm{s}$ & Maximum sensor spacing & $s=l_{m}+w_{m} \tan \theta$ & $3.1 \mathrm{~m}$ \\
\hline
\end{tabular}

\subsubsection{Computer Vision Sensor Modeling}

In order to use computer vision to recognize vehicles accurately in real-time, appropriate hardware coupled with a fast robust algorithm must be used. Popular methods used for object detection requires using specialty cameras (i.e. infrared) or implementing stereoscopic vision. 
However, those methods are expensive and require substantial processing capability. A low-cost low-processor system is a more appropriate solution for this project.

Based on the real-time requirements and available hardware, several image processing techniques have been selected as potential methods of detecting vehicles.

\section{Edge based shape detection}

Most vehicles are rectangular in nature. Captured frames can be filtered using an edge detection algorithm such as the Sobel or Canny. A shape detection algorithm such as the Hough transform follows that, allowing objects to be digitized and tracked in subsequent frames. Depending on the image resolution, the edge detection algorithm and the number of detected objects, the algorithm may require substantial processor power.

\section{Frame comparison}

By comparing the previous captured frame with the current frame and executing a pixel-by-pixel intensity subtraction, the differences between frames can be detected. This can be used to either filter out stationary objects when the host vehicle is not moving, or to detect moving objects that are moving behind the host vehicle. This is merely an intermediate filtering step used to reduce as much stationary terrain in the image as possible, resulting in less noise. Using this method in conjunction with an edge based detection method yields more accurate vehicle recognition results.

\section{Edge based color detection}

Under normal daylight conditions, a vehicle's color can be used for detection. The captured image is masked into a strip to reduce processing requirements. The frame undergoes color and edge detection. Comparing data from previous captured frames, vehicle location and relative 
distance can be determined. Figure 10 depicts two successive frames and edge tracking for the green vehicle.
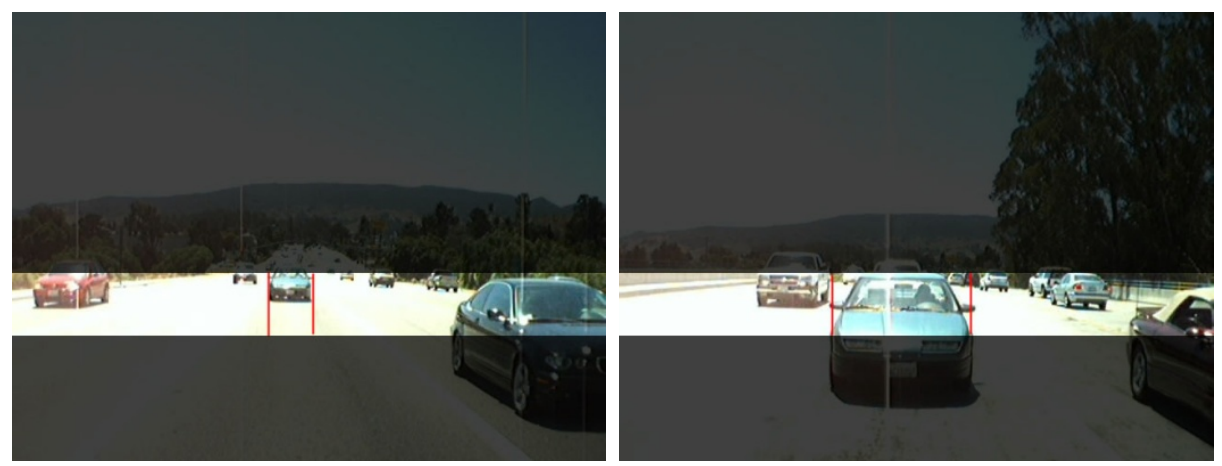

Figure 10. Edge based color detection example.

\section{Headlight detection}

During evening or low-light conditions, a vehicle can be tracked using headlight detection.

Similar to color detection, the captured image will be masked and the intensity would be used to track the vehicle.

\section{Object discrimination}

A digital camera is mounted on the rear of the test vehicle. Data transfers through FireWire (IEEE 1394) to a laptop. An external power source currently powers the camera, but a 6 pin-6 pin

FireWire can supply the necessary power. Matlab running on the laptop handles low level FireWire interfacing as well as image processing tasks.

The image processing algorithm developed focuses on shape detection (Fig. 11).

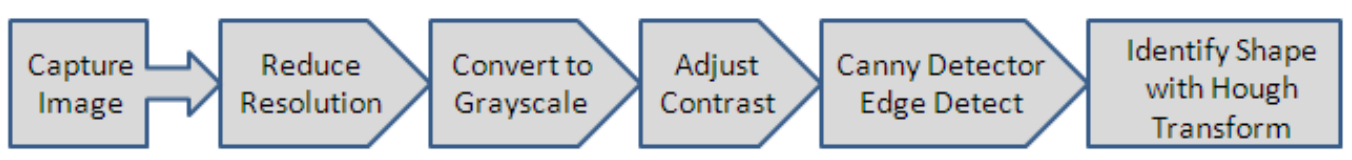

Figure 11. Image processing algorithm.

Captured video footage of different sized vehicles driving on a freeway is fed to the algorithm to see how effective it is in detecting vehicles and filtering out terrain. 
The vision algorithm was found to be very processor intensive and refinements to its processing methods were considered to increase the accuracy of vehicle detection in real time. In order to decrease processing time, the image resolution was quartered (dimensions halved) and an edgebased color detection method was considered.

Due to the high development time required to implement a reliable computer vision system, the computer vision is only considered to be a secondary system used as a reference to verify the presence of vehicles and is not an integral part in the crash avoidance algorithm.

\subsection{BASELINE TESTING}

Baseline testing was conducted on the magnetic, radar, and ultrasonic sensors to establish critical performance characteristics. Testing was conducted first in a lab workbench environment, then in a static traffic environment and finally in a moving traffic environment. Baseline testing was not conducted on the vision sensor because it was concluded this sensor is not suitable for the proposed crash avoidance algorithm (Section 2.4.3); however, the sensor is still used to reference the presence of objects detected by the other sensors.

\subsubsection{Magnetic Sensor Testing}

The experimental study was carried out in two parts. The first part was a laboratory test that utilized a bench top unit (Fig. 12) while the second part consisted of road tests.

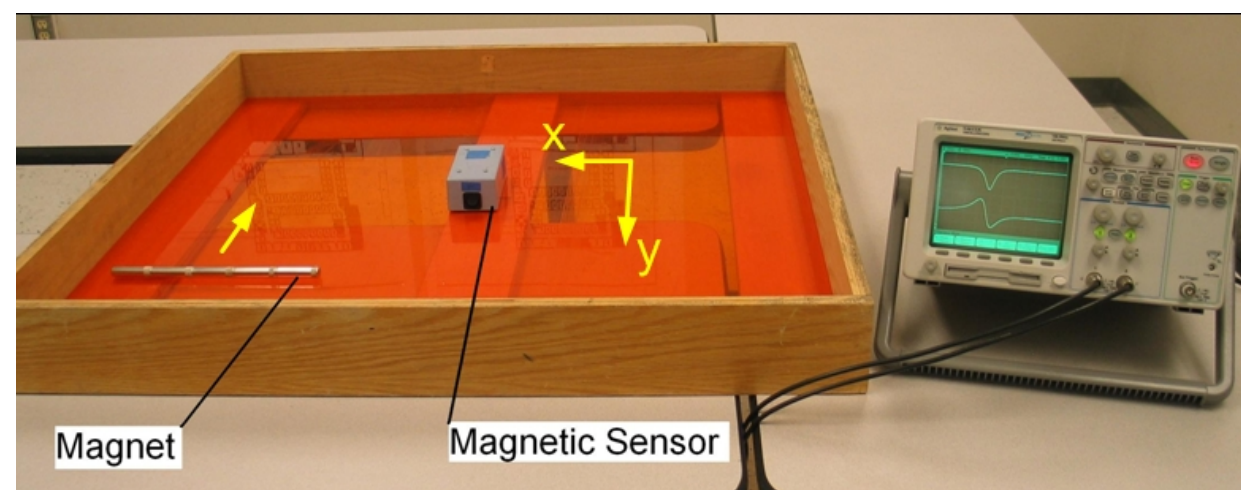

Figure 12. Laboratory bench test apparatus. 


\section{Laboratory Bench-Tests}

Literature suggests that a vehicle can be modeled by a single magnetic dipole [17]. Accordingly, in lab bench tests, permanent magnets were used to simulate the effects of vehicles. To gain a first-hand feel for the working of the magneto-resistive sensor, the preliminary bench test apparatus was constructed as shown in Fig. 12. The sensor electronics were enclosed in a plastic casing and placed at the center of the apparatus. Two different sized axially magnetized cylindrical permanent magnets were used to simulate the presence of cars and trucks. The magnets were moved parallel to the y-axis of the sensor (see Fig. 12) to simulate a vehiclepassing scenario. The induced magnetic field from the two different magnets was then recorded by the magnetic sensor and is as shown in Fig. 13.
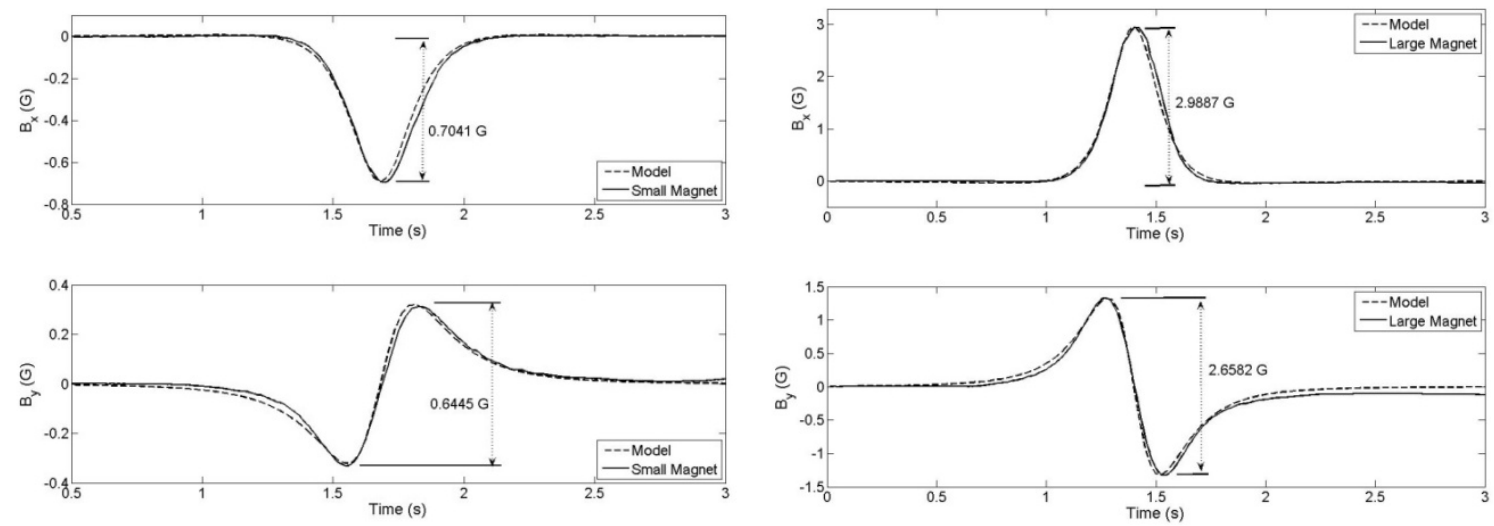

(a) Small magnet.

(b) Large magnet.

Figure 13. Magnetic sensor bench test results.

\section{Road tests}

Based on the encouraging results obtained from the bench tests and the theoretical corroboration of the sensor working-principle, the magnetic sensor was tested on the road. Figure 14 shows a schematic of the road test configuration. The sensor was mounted on the lateral side of the vehicle with its sensitive axes oriented in the directions shown. Test vehicles were made to pass the sensor at a constant velocity in the direction shown and their magnetic footprints were recorded in all the three dimensions $\mathrm{x}, \mathrm{y}$ and $\mathrm{z}$ by the onboard data acquisition system. Figure 15 
shows two such profiles recorded for a typical passenger car and a class 8 semi-truck/trailer respectively. It can be observed from these figures that unlike the bench-tests which used single dipole magnets, the magnetic profiles for both the car and the truck showed the superposition of multiple single dipoles. The presence of multiple dipoles can be seen in both magnetic footprints in Fig. 15 by the number of peaks that are present above 0 Gauss.

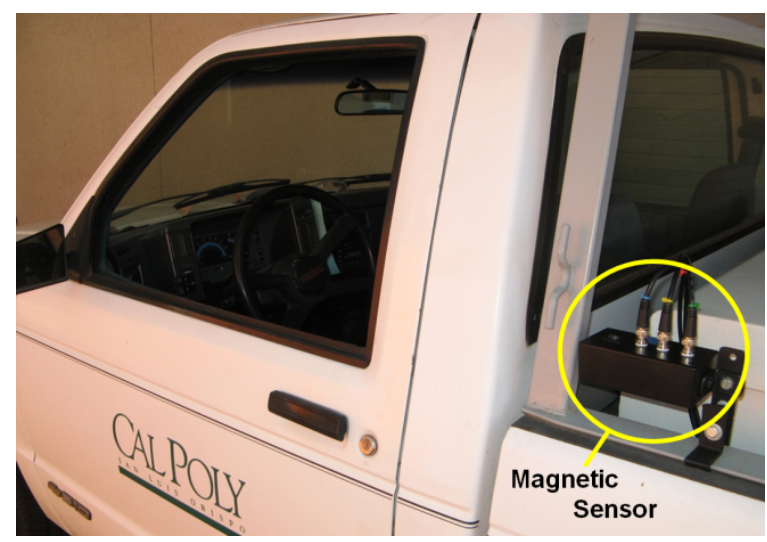

(a) Magnetic sensor mounted on test vehicle

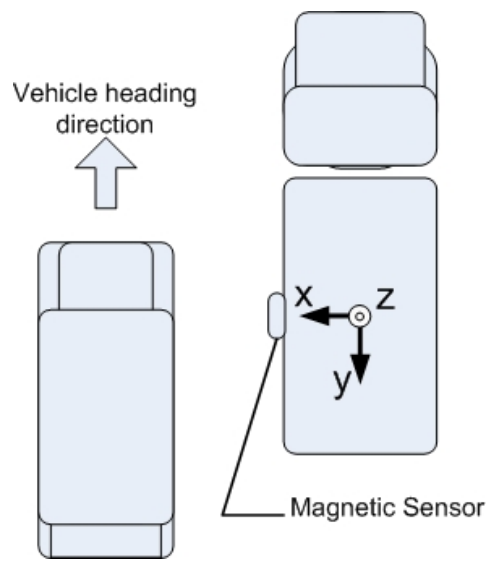

(b) Magnetic sensor orientation

Figure 14. Magnetic sensor road test configuration.
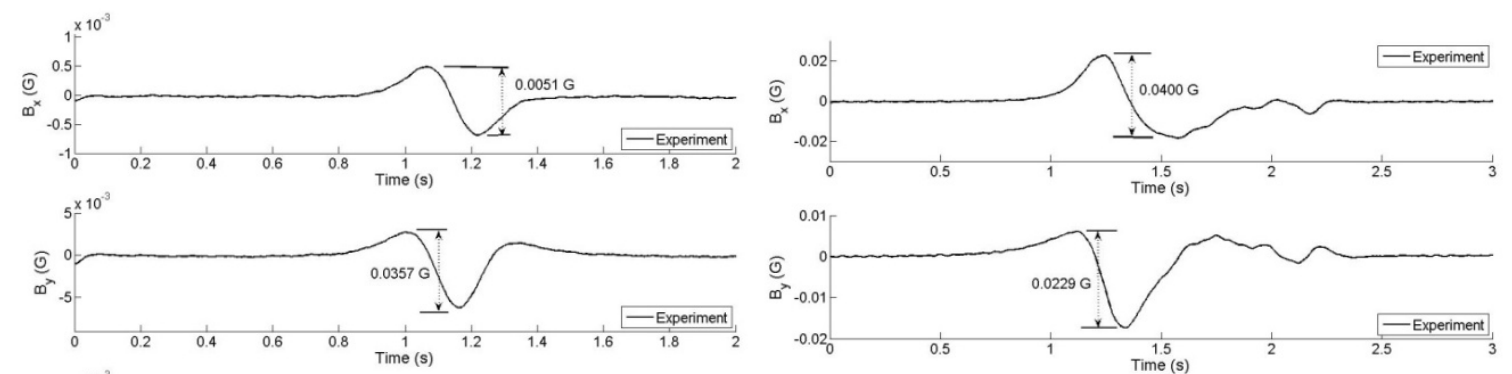

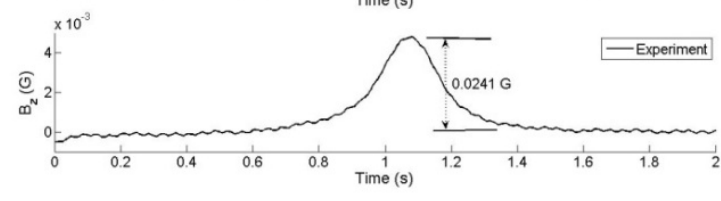

(a) Passenger car.

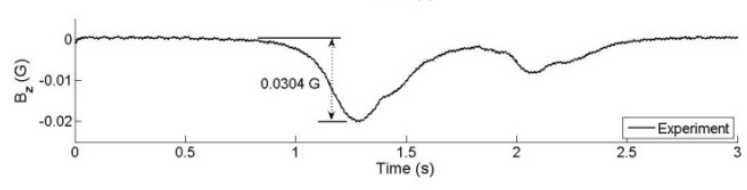

(b) Class 8 truck.

Figure 15. Magnetic sensor road test result.

\subsubsection{Radar Sensor Testing}

For this project, a $24 \mathrm{GHz}$ Ultra-Wide Band Short Range Radar was procured that is widely used for Adaptive Cruise Control (ACC) applications in the automotive market. The radar is capable of rapid detection of multiple objects, can provide quick information on both stationary and moving 
objects (distance, relative velocity, and bearing), and is functional in adverse weather conditions (e.g. rain, snow, fog, etc...). In order to confirm the radar specifications provided by the supplier and to become familiar with its operation and capabilities a series of bench tests and road tests were performed:

- Identify the noise floor - Determined if the radar reports spurious results when viewing a field of view with no objects, to investigate the occurrence of false positives.

- Angle Beamwidth - Computed the azimuth and elevation fields of view using simple trigonometry (see Fig. 16). The target was kept at a known distance, $d$, from the face of the radar and it is moved in the negative $x$ direction until detected by the radar; at this point the distance $a$ was measured and used along with $d$ to compute $\theta$.

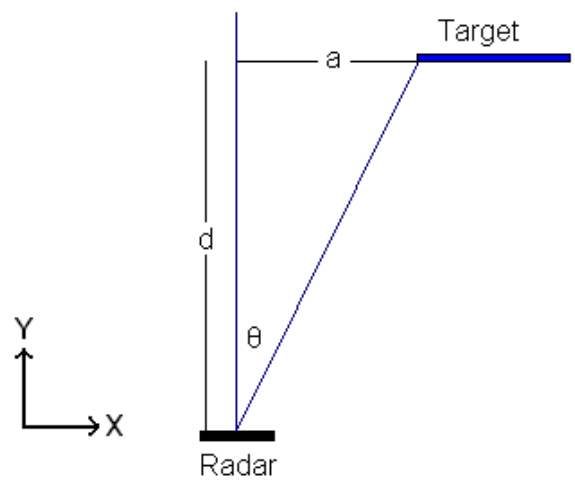

Figure 16. Schematic of radar azimuth and elevation angle measurements.

- Detection Range - Determined the detection range by keeping track of a target as it moved away from the radar until no longer observed. When the radar was unable to locate the target the detection range was thus determined.

- Range Accuracy - Determined the accuracy of the radar range measurements by doing a controlled experiment where targets were placed at known distances and the radar was used to measure the distances to the targets.

- Target Detection Bench Tests - These tests consisted of placing different size and shape objects in the vicinity of the sensor and arranging them in different order and distances relative to each other. The purpose of these tests was to determine what the radar considers a target; does it combine multiple objects into a single target or does it give multiple targets for a single object? 
- Target Detection and Tracking Road Tests - The tests consisted of placing the radar in the rear bumper of a truck facing backward to tag targets and keep track of them. The following scenarios were considered: (a) a target vehicle stays behind the host vehicle. (b) a target vehicle approaches from behind and merges into the left lane. (c) The host vehicle passes a target vehicle on the right. These tests evaluated the tagging and tracking capabilities of the radar.

The Target Detection and Tracking road test were conducted using the same passing scenario used in the ultrasonic and magnetic sensor tests (Fig. 17).
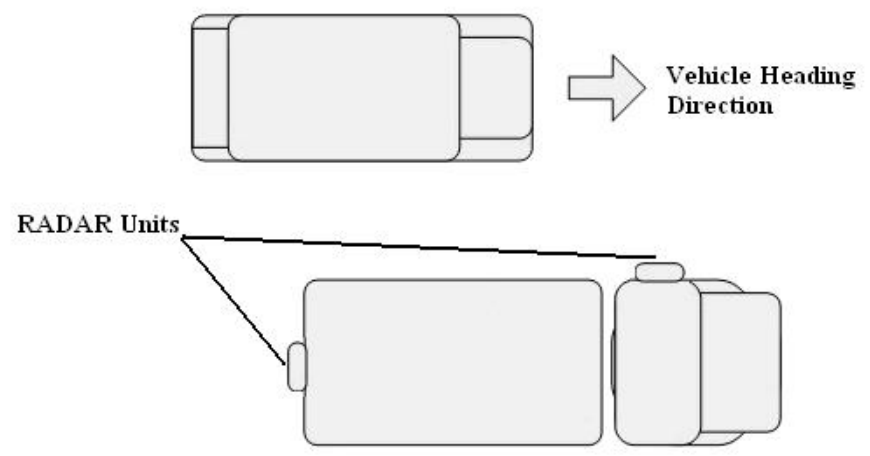

Figure 17. Radar road test schematic.

The radar accurately tracked the moving vehicle while providing range, velocity, and bearing information. Figure 18 shows a passing vehicle as it approaches the host vehicle from the rear.
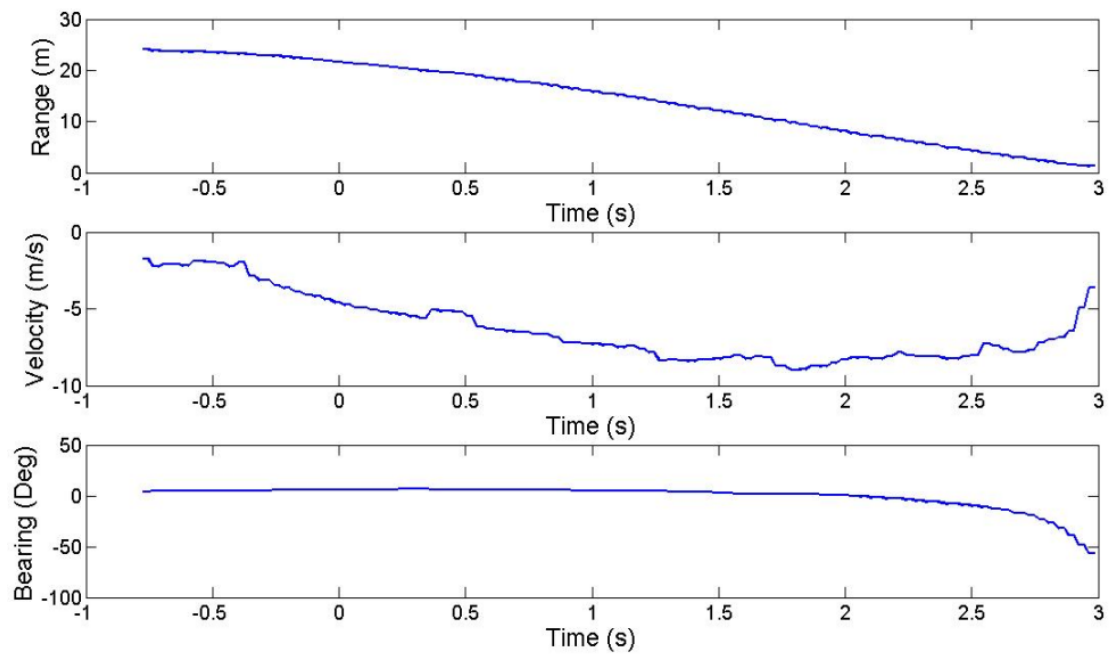

Figure 18. Radar (rear facing) road test results. 
. When identifying the noise floor false positives were observed but the radar was unable to keep track of these false positives - giving a good method to discard them (lost tagged objects can be ignored). Finally, the bench tests for Target Detection showed very promising results. The radar lumps object that are close to each other into a single target but is able to distinguish between two objects that are further apart. Vehicle distances on the highway are significantly greater than those used in the bench test, indicating that the radar should be able to track vehicles individually.

Based on these results, the radar has good potential as one of the main sensors for this project.

This radar was subsequently positioned at the rear of the class 8 trailer to identify and tag vehicles as they approached. By knowing the location, velocity and bearing of an approaching vehicle the algorithm should be able to predict its future location and be ready to track it with the sidemounted sensors.

\subsubsection{Ultrasonic Sensor Testing}

For testing purposes, the $42 \mathrm{kHz}$ LV-MaxSonar®-EZ1 ultrasonic sensors were enclosed in plastic enclosures fitted with mounting brackets, wind shield, and powered by a $9 \mathrm{~V}$ battery and a $5 \mathrm{~V}$ voltage regulator. Figure 19 shows the ultrasonic sensor housing as well as its orientation on a test vehicle.
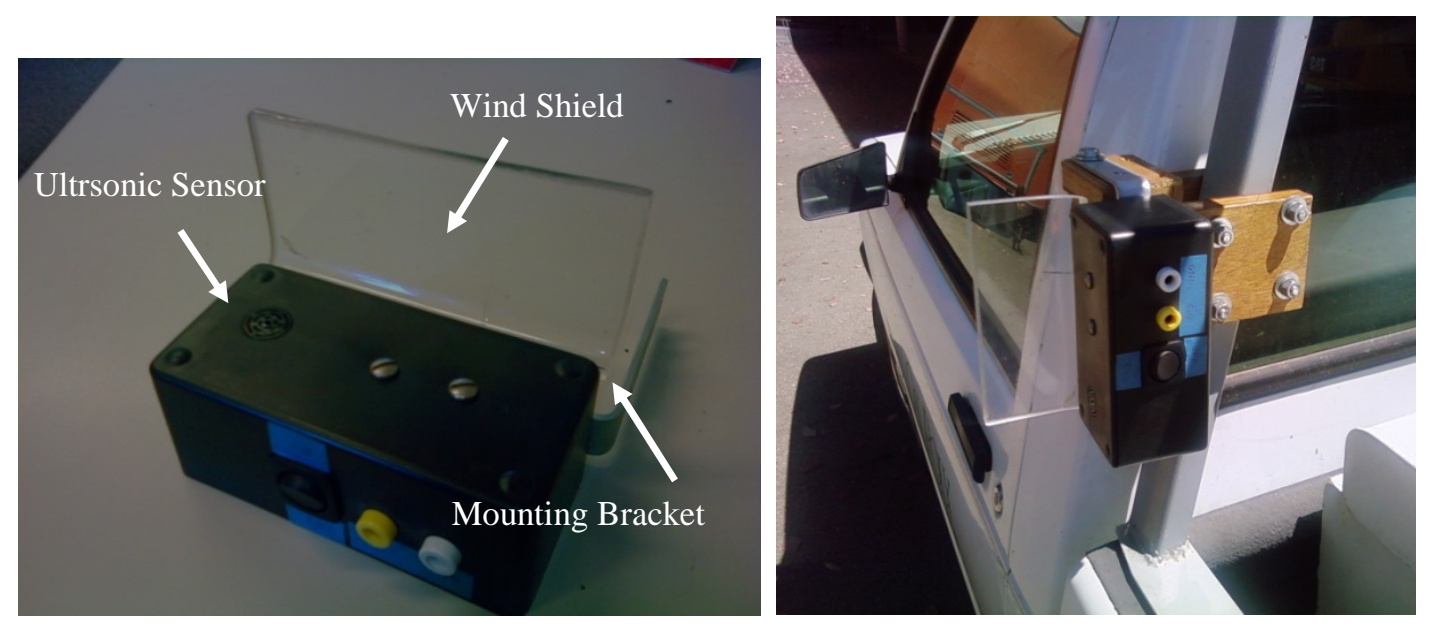

Figure 19. Ultrasonic sensor enclosure. 


\section{Laboratory Study}

The operation and function of ultrasonic sensors are well known, thus laboratory testing focused on profiling the beam characteristics and voltage to distance relationship for this particular sensor. An ultrasonic sensor beam characteristic can vary depending on the size, curvature, and material of the object it is detecting. Representing the material of a moving vehicle, a 0.5 m metal flat plate was used to profile our sensor's beam contour. This plate was moved left to right while parallel to the sensor face and with the sensor stationary. Figure 20 shows the beam characteristic of our ultrasonic sensor.

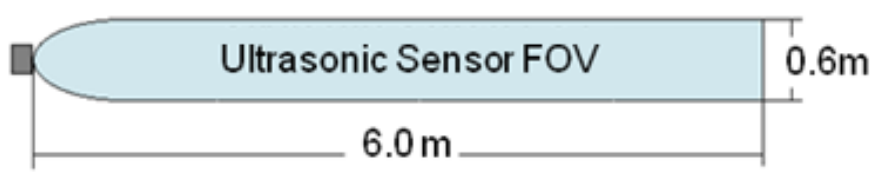

Figure 20. Ultrasonic sensor beam characteristic.

The largest error created from a target measured at the edge of the beam profile rather than the centerline is $0.12 \%$ at $6 \mathrm{~m}$.

Using the same target as before, the relationship between the sensor output voltage, input voltage, and range along the sensor's centerline was found to be $V=\frac{V_{c c}}{1300} \cdot d$ where $\mathrm{d}$ is range $(\mathrm{cm})$ and $V_{c c}$ is the sensor input voltage, regulated at 5 volts.

\section{Mobile Testing}

Although ultrasonic sensors are used in current vehicles, their use is restricted to low-speed applications. Other researchers have found that these sensors may become inaccurate at vehicle speeds of $30 \mathrm{~km} / \mathrm{hr}$ [8] to $105 \mathrm{~km} / \mathrm{hr}$ [7]. To corroborate this data with our sensor, wind tunnel testing was considered to verify the operating range of our ultrasonic sensor. However, due to the proximity of the tunnel walls the operating conditions of the sensor could not be replicated. 
Instead, field tests were used: The ultrasonic sensor was attached to a test fixture on a light vehicle and faced out perpendicular to the length of the vehicle (See Fig. 21). Effects of vehicle speed or wind flowing perpendicular to the sensor face caused significant sensor output noise around $80 \mathrm{~km} / \mathrm{hr}$. Other wind turbulence such as in high wind gust areas created the same noise. By employing shielding (adding a solid surface to direct the airflow around the sensor - see Fig. 21) the turbulence over the sensor was reduced, allowing the sensor to operate effectively at highway speeds ( $65 \mathrm{mph}$ or $105 \mathrm{~km} / \mathrm{hr}$ ). The same noise can further be reduced by installing the ultrasonic sensor at an angle facing away from the direction of wind flow rather than facing the sensor perpendicular to the vehicle (Fig. 9).

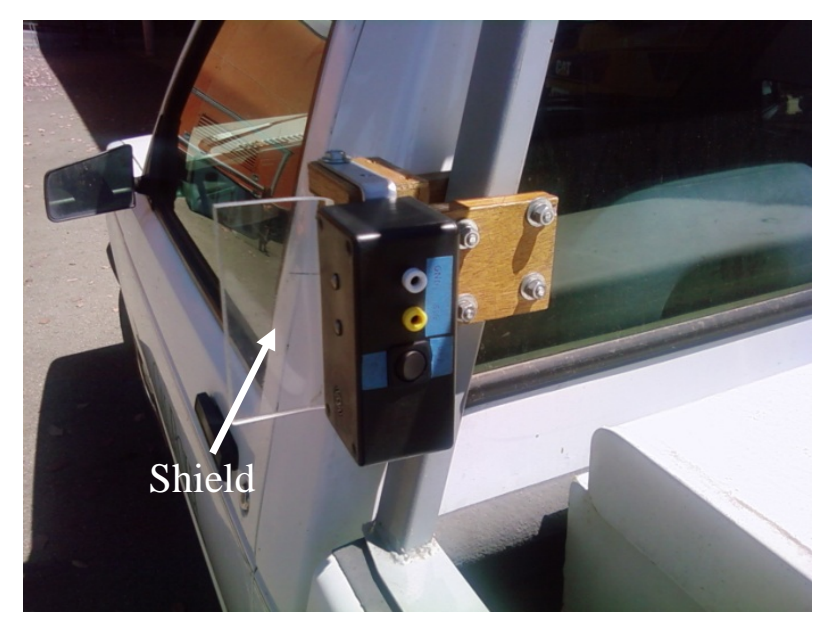

Figure 21. Ultrasonic sensor mobile test setup.

\subsection{SIMULATIONS}

Simulations were conducted to compare the magnetic sensor mathematical models with the testing to validate the models. It was decided that the radar and ultrasonic sensors were well understood and established for similar applications, so further simulations of these sensors were not necessary.

The simple 2-D single dipole model (5) and (6) was used to perform simulations replicating the bench tests that were conducted. Using appropriate parameter values for the dipole length, dipole angle and empirical relations $\left(K_{x}\right.$ and $\left.K_{y}\right)$, the response was obtained and compared with the 
experimental results. Figure 22 shows a good match between theory and experiment justifying the ability of the model to predict the magnetic phenomenon recorded in experiments.
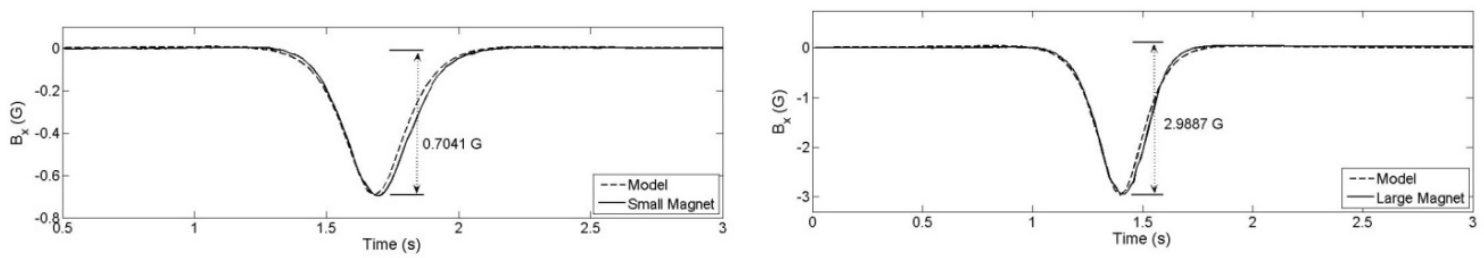

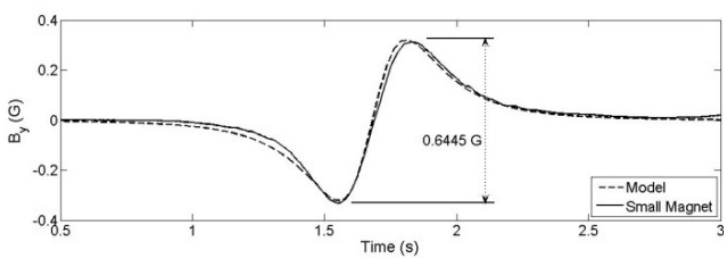

(a) Small magnet.

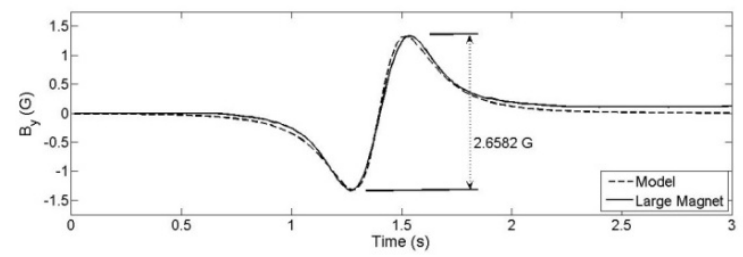

(b) Large magnet.

Figure 22. Comparison of simple dipole model experiment and simulation

\section{Magnet Type Detection}

From Eqn. (1) it can be seen that the magnetic sensor output depends of the strength of the induced magnetic field, which in turn is dependent of the size of the magnet. Hence, the magnetic sensor can possibly be used to detect the magnet size. Figure 23 shows a clear distinction (from a magnitude standpoint) between the magnetic fields induced by the two different magnets, thereby corroborating this claim. However, these magnetic fields are sign dependent and flip sign when the dipoles are flipped. Hence, in order to clearly distinguish the different sized magnets, appropriate mathematical functions that not only eliminate this sign dependency, but also produce a pronounced difference in the values were studied. Two such functions are,

$$
\begin{gathered}
\left|B_{x}\right|+\left|B_{y}\right| \\
\left(B_{x}\right)^{2}+\left(B_{y}\right)^{2}
\end{gathered}
$$

The results obtained by using these functions are as shown in Fig. 23. Both the functions were able to achieve the desired objective and the sum-square function in particular was able to provide a more comprehensive threshold difference that could be used to clearly distinguish the smaller magnet from the bigger one. 
Figure 24 shows a comparison between the mathematical model response and the experimental response from the road tests. The high fidelity 3-D mathematical model comprised of two dipoles for modeling the effect of cars, and five dipoles to obtain an accurate representation of typical class- 8 trucks. The following section describes the use of mathematical functions on the magnetic data to help distinguish different vehicle types.
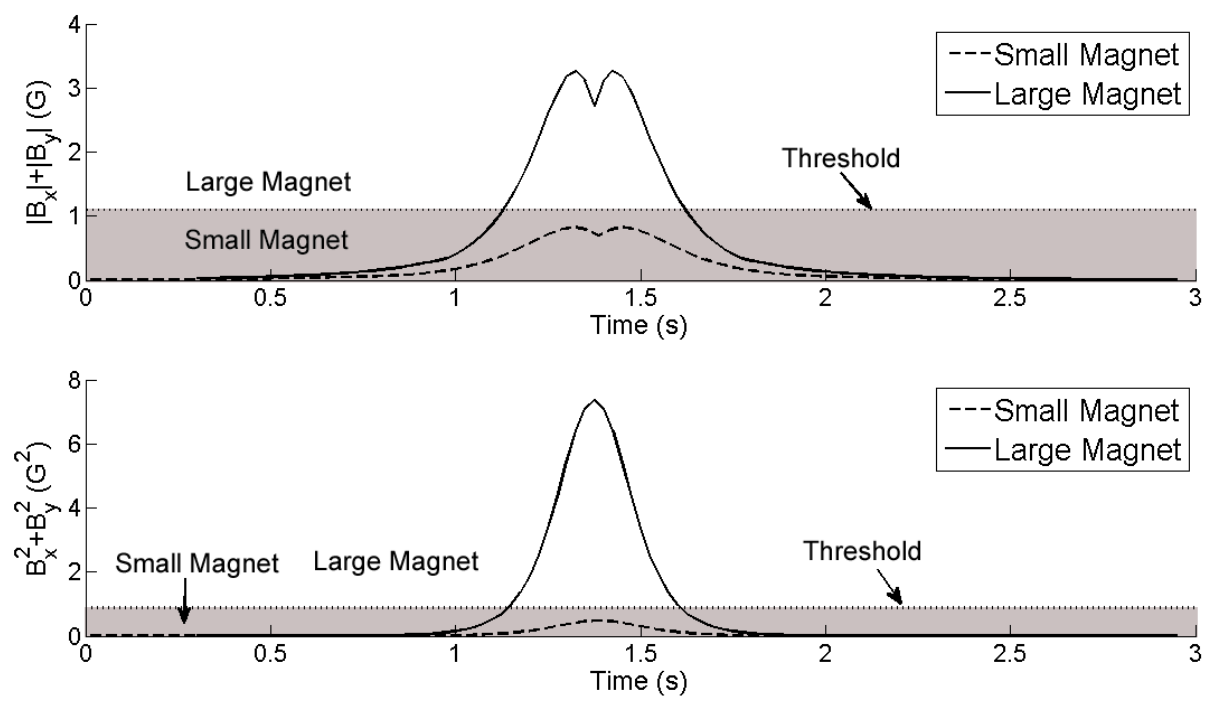

Figure 23. Magnetic threshold for object discrimination for simple dipoles.
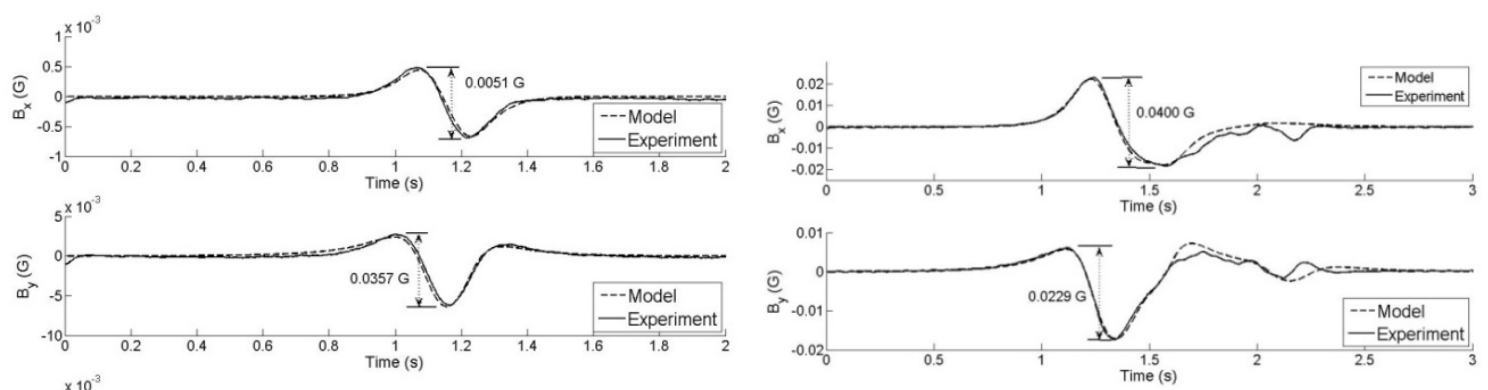

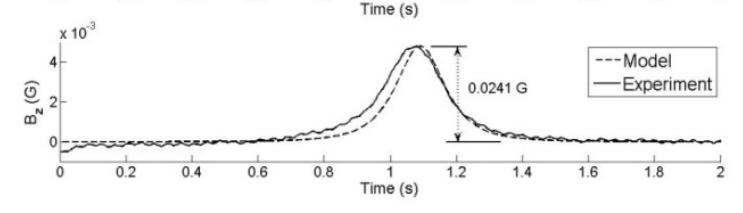

(a) Passenger car.

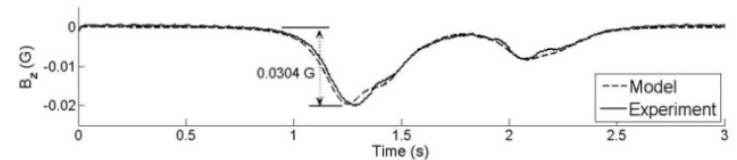

(b) Class 8 truck.

Figure 24. Comparison of on-road simulation and road test. 


\section{Vehicle Type Discrimination}

Similar to the case of the 2-D model, mathematical functions were employed to not only extract the magnitude information, but also magnify the combined magnetic effects along all three axes of measurement. Two functions employed were

$$
\begin{gathered}
\left|\mathrm{B}_{\mathrm{x}}\right|+\left|\mathrm{B}_{\mathrm{y}}\right|+\left|\mathrm{B}_{\mathrm{z}}\right| \\
\left(\mathrm{B}_{\mathrm{x}}\right)^{2}+\left(\mathrm{B}_{\mathrm{y}}\right)^{2}+\left(\mathrm{B}_{\mathrm{z}}\right)^{2}
\end{gathered}
$$
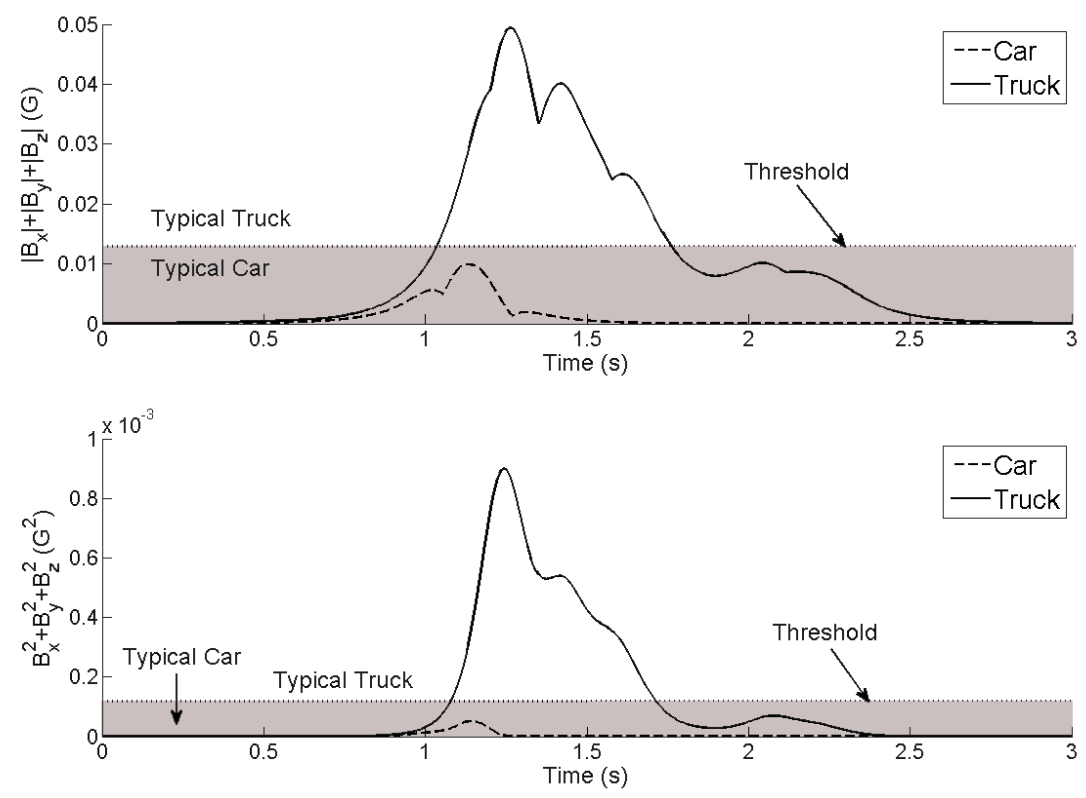

Figure 25. Magnetic threshold for object discrimination for vehicles.

From Fig. 25 it can be seen that there exists a clear threshold in the magnitude obtained for a typical truck when compared with that for passenger car. It can also be observed that the sumsquare function is able magnify this threshold to a much greater extent. The extensive modeling, testing, and simulations performed with the magnetic sensor have confirmed its capabilities for object type discrimination. 


\section{VEHICLE DETECTION ALGORITHM}

Radar, ultrasonic sensors, and magnetic sensors were identified as suitable sensors to be used in this crash avoidance system. Through modeling, simulation, and baseline testing the strengths and weaknesses of these sensors were identified. Because of the environmental and process noise inherent in monitoring a region around a vehicle, the data from these individual sensors are not reliable enough to make irreversible decisions. An intelligent algorithm is required to integrate data from the multiple sensors to create a situational awareness within the system that is far greater than the individual sensors can provide, at a price far less than a new custom-designed sensor.

\subsection{ALGORITHM DEVELOPMENT}

An intelligent algorithm is required to translate sensor data into useful information for a crash avoidance system. It is necessary for any warning or countermeasure taken by a crash avoidance system to be completed as soon as possible without false positives or overreacting in the situation. Simply interpreting the sensor data is not sufficient to identify threats because these sensors usually carry data that is noisy or incomplete. It is crucial that any sensor noise created by the environment (especially that of a large truck) is considered and reinforced by other sensor information. A probabilistic approach has been taken to help manage measurement of uncertainty and perform multi-sensor fusion. The following sections discuss the basic concepts used in probability, the basic concepts of Bayesian filtering and its uses, how this Bayesian filtering may be applied to vehicle identification, and how this technique facilitates sensor fusion.

\subsubsection{Basic Concepts in Probability}

For this application, voltage measurements taken from individual sensors are treated as random variables. Let the random variable (sensor measurement) be denoted by the variable $Z$ and the specific sensor reading at time t be denoted as $z_{t}$. These random variables can take on multiple 
values, and they do so according to specific probabilistic laws. A probabilistic law can be defined for sensor measurements for specific applications, such as vehicle detection and is explain in the next section.

To describe the probabilistic nature of sensor data, it is assumed that they possess probability density functions (PDFs). It is common for sensor PDFs to be that of the one-dimensional normal distribution with mean $\mu$ and variance $\sigma^{2}$. Normal PDFs are given by the following Gaussian function [21]:

$$
p\left(z_{t}\right)=\left(2 \pi \sigma^{2}\right)^{-\frac{1}{2}} \exp \left\{\frac{-(z-\mu)^{2}}{2 \sigma^{2}}\right\}
$$

where $p(z)$ represents the likelihood of a sensor measurement $z$ with an expected value of $\mu$ and the variability of the sensor measurement represented by $\sigma^{2}$. The PDF for the ultrasonic sensor and magnetic sensor are derived later in "Statistical Sensor Modeling" and used to attain the likelihood of vehicles being present around the host vehicle while taking into account false targets.

The information from an individual sensor can be compared with data from other sensors when applying a probabilistic approach for multiple sensors; this process is called joint distribution. Joint distribution describes the probability that the random variable $X=x$ and that $Y=y$. If $X$ and $Y$ are independent the joint distribution is given to be

$$
p(x, y)=p(x) p(y)
$$

Joint distribution is important for multi-sensor fusion in vehicle detection because presence of an object and its type is difficult to positively identify with a single sensor. The above equation can be used to integrate multiple sensors because the information of each sensor is independent of the other. Figure 26 shows the results of joint distribution between two sensor belief curves. If two sensors are in agreement, the joint likelihood has a unique mode (the value that occurs most 
frequently) at the estimated state variable; however, when the sensors are in disagreement, the joint likelihood is bimodal and has a low likelihood at the estimated state variable. This idea of joint distribution can be applied to the ultrasonic sensors and magnetic sensors to check for the presence of an object and to ascertain if the object is a vehicle.

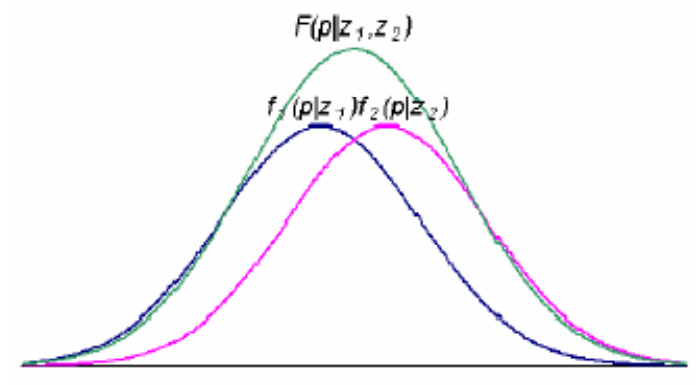

(a) Joint likelihood of two sensors in agreement.

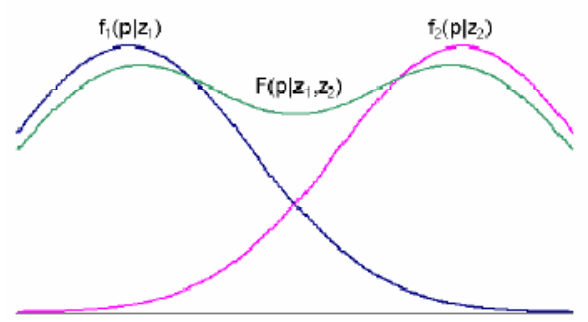

(b) Joint likelihood of two sensors in disagreement.

Figure 26. Joint likelihood of two sensors [22].

Often, random variables carry information about other random variables and thus are not independent. The joint distribution of the two random variables is then called conditional probability, which can be stated as the probability of $X=x$ being true when $Y=y$ is true. From [21] conditional probability is denoted as

$$
p(x \mid y)=\frac{p(x) p(y)}{p(y)}
$$

Conditional probability can be used to describe the behavior of an individual sensor or the likelihood of an event by assuming that the measurement at time $t$ is dependent on the measurement at $t$ - 1 . Conditional probabilities can also be examined by an alternate method called Bayes rule [21].

$$
p(x \mid y)=\frac{p(y \mid x) p(x)}{p(y)}
$$

"If $x$ is a quantity that we would like to infer from $y$, the probability $p(x)$ will be referred to as prior probability distribution, and y is called the sensor measurement data. The distribution $p(x)$ summarizes the knowledge we have regarding $X$ prior to incorporating the data $y$. The probability $p(x \mid y)$ is called the posterior probability distribution over $X$. This method provides a convenient way to compute posterior conditional probability $p(x \mid y)$ using the "inverse" conditional probability $p(y \mid x)$ 
along with the prior probability $p(x)$.In other words, if we are interested in inferring a quantity $x$ from sensor data y, Bayes rule allows us to do so through the inverse probability, which specifies the probability of the data y assuming that $x$ was the case." [21]

The following section expands on the basic concepts of probability and explains how Bayes rule can be modified into a filter to reject sensor noise. Furthermore, this filter is modified to handle information from the ultrasonic and magnetic sensors and play an active role in the proposed vehicle detection algorithm.

\subsubsection{Bayesian Filtering}

Raw data from sensors is corrupted by process noise and anomalies due to environmental influences as seen in Fig. 27. In this figure, the ultrasonic sensor voltage indicates the presence of a vehicle when the voltage is below $1 \mathrm{~V}$. The noise outlined in red in Fig. 27can cause a false negative reading in a vehicle detection algorithm and must be accounted for. Bayesian filters can be created to filter this noisy or partial sensor data using the basic concepts in probability from the previous section [21], [23]. A Bayesian filter is a recursive state estimation model with the ability to output the likelihood of an event occurring. The state of the surroundings around sensors cannot be measured directly due to environmental and process noise; however, the likelihood of the state can be inferred through sensor data and a Bayesian filter. The filter is completed in two steps: the prediction step and correction step.

Prediction Step: At each time update, the state is predicted according to the following update rule [21], [24].

$$
\operatorname{Bel}^{-}\left(x_{t}\right)=\int p\left(x_{t} \mid x_{t-1}\right) \operatorname{Bel}\left(x_{t-1}\right) d x_{t-1}
$$

The predicted belief of the state variable at time $t, \operatorname{Bel}^{-}\left(x_{t}\right)$, is represented by the integral or sum of the product of two distributions: the prior distribution, $\operatorname{Bel}\left(x_{t-1}\right)$, and a predicted belief based on the prior belief. The term $p\left(x_{t} \mid x_{t-1}\right)$ describes the system dynamics, which ascertains how the state 
of the system changes over time. This term predicts the likelihood of the system state based on the last measurement. The prediction parameters are described in the following section.

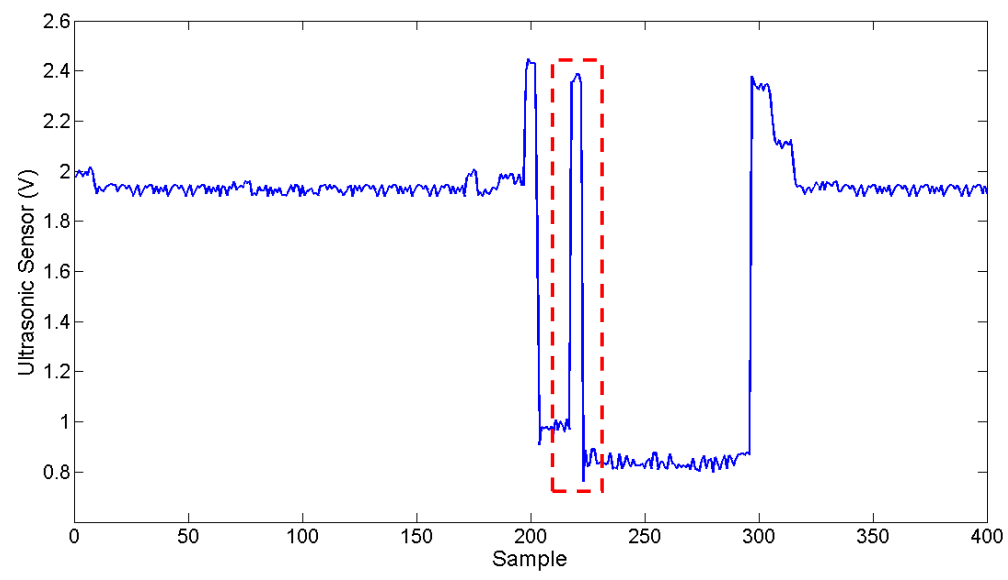

Figure 27. Raw ultrasonic data with noise.

Correction Step: Whenever new sensor information $z_{t}$ is received, the measurement is used to correct the predicted belief using the observation [21], [24].

$$
\operatorname{Bel}\left(x_{t}\right)=\eta p\left(z_{t} \mid x_{t}\right) \operatorname{Bel}^{-}\left(x_{t}\right)
$$

The term $p\left(z_{t} \mid x_{t}\right)$ is the perceptual model that describes the likelihood of making observation $z_{t}$ given that a state variable is equal to $x_{t}$. For location estimation, the perceptual model is usually considered a property of a given sensor technology. It depends on the types and positions of these sensors and captures a sensor's error characteristics. The term $\eta$ is a normalizing constant which ensures that the posterior over the entire state space sums up to one. This constant is discussed in more detail in the following section.

\subsubsection{Bayesian Filter Algorithm}

Bayesian filtering can be directly applied to the sensors for the purposes of vehicle detection. To clearly explain how the Bayes filter algorithm is developed; consider only the ultrasonic sensor with the state variable of interest being the presence of an object. This procedure will later be 
expanded to include the magnetic sensor and other state variables. As mentioned in the previous section, the Bayesian filter is completed in two steps: prediction and correction.

Prediction Step: The predicted model for the ultrasonic sensor is based on Theorem of Total Probability. The following equation represents the predicted probability of an object's presence at time $t$ based on the probability of an object's presence at time $t-1$ [21].

$$
\left.p\left(x_{t}^{\prime}\right)=p\left(x_{t}^{\prime} \mid x_{t-1}\right) p\left(x_{t-1}\right)+p\left(x_{t}^{\prime} \mid\right\urcorner x_{t-1}\right) p\left(7 x_{t-1}\right)
$$

Here, the terms $p\left(x_{t}^{\prime} \mid x_{t-1}\right)$ and $\left.p\left(x_{t}^{\prime} \mid\right\rceil x_{t-1}\right)$ describe the predicted probability that an object is present at time $t$ based on the probability that an object is present at time $t-1$ and the probability that an object is absent at time $t-1$ respectively. In detecting an object's presence, this conditional probability is referred to as the motion model where the vehicle might be at time $t$, given its location at $x_{t-1}$.

Correction Step: Using the information from the prediction step, the likelihood of a vehicle's presence $p(x)$ and a vehicle's absence $p(7 x)$ are evaluated using the correction step. The correction step of the algorithm is represented by [21]:

$$
\begin{gathered}
p(x)=\eta p\left(z_{t} \mid x_{t}^{\prime}\right) p\left(x_{t}^{\prime}\right) \\
\left.p(7 \mathrm{x})=\eta p\left(z_{t} \mid\right\urcorner \mathrm{x}_{\mathrm{t}}^{\prime}\right) p\left(7 \mathrm{x}_{\mathrm{t}}^{\prime}\right) \\
\eta=\left[p\left(z_{t} \mid x_{t}^{\prime}\right) p\left(x_{t}^{\prime}\right)+p\left(z_{t} \mid 7 \mathrm{x}_{\mathrm{t}}^{\prime}\right) p\left(7 \mathrm{x}_{\mathrm{t}}^{\prime}\right)\right]^{-1}
\end{gathered}
$$

where $\eta$ represents the normalizing parameter to ensure the probability of a $p(x)$ and $p(7 x)$ are between 0 and 1 . 


\subsection{SYSTEM TESTING}

For algorithm development, system testing was completed in three stages: preliminary data collection, simulation, and a full scale test. The data collection was performed using a small test vehicle with the various sensors attached. Data collected from this test vehicle was used to create the sensor probability density functions explained in the previous section. The data collected was post processed and used to simulate the effectiveness of a Bayes filter algorithm. Finally, the system was attached to a heavy truck and data was collected and applied to the purposed algorithm.

\subsubsection{Data Collection}

The test vehicle seen in Fig. 28 was modified to include two ultrasonic sensors, two magnetic sensors, and two radars. Information was collected from all sensors as vehicles passed the lateral side of the vehicle. A digital camera was used as reference to positively identify the presence of a passing vehicle (not shown in figure).

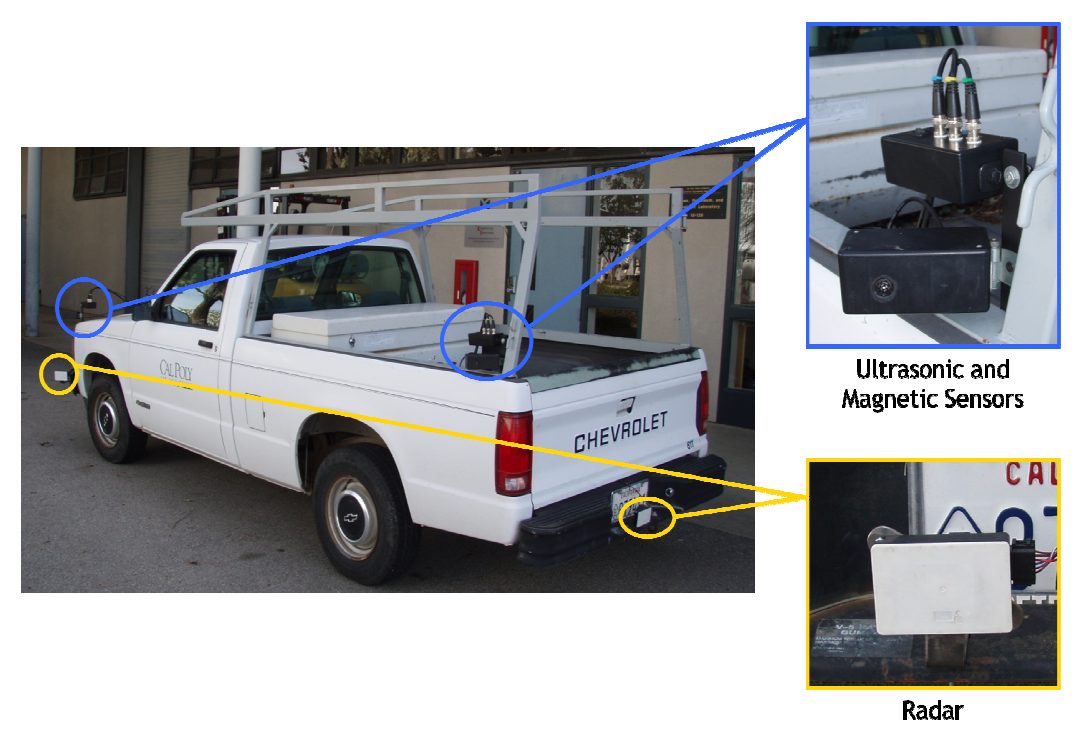

Figure 28. Test vehicle (pickup truck).

Figure 29 shows the typical data set for a passing vehicle. As the vehicle passes, three distinct regions based on the vehicle's location in reference to the sensors are taken into consideration: 
region $\mathrm{A}, \mathrm{B}$, and $\mathrm{C}$. It is clear that Region $\mathrm{B}$ has the most potential to positively identify the presence of a vehicle.

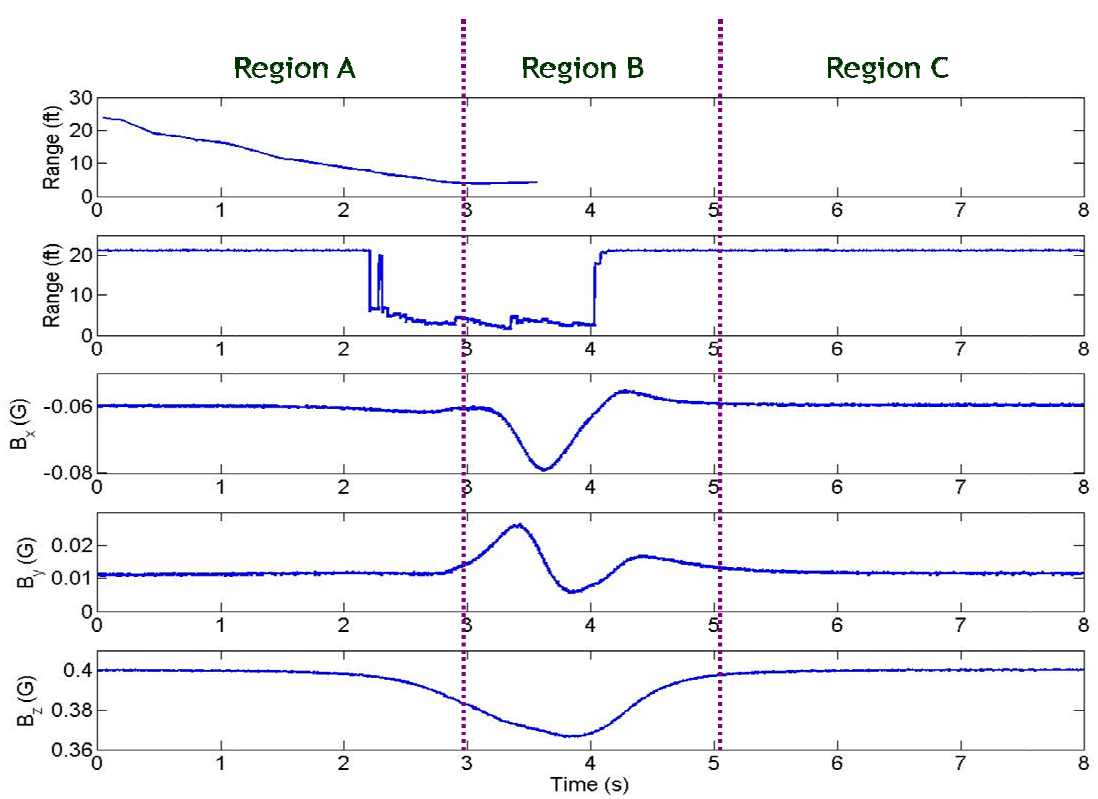

Region A: Car in adjacent lane approaching rear sensors of test vehicle. Region B: Car in adjacent lane beside sensors of test vehicle. Region C: Car in adjacent land ahead of sensors.

Figure 29. Sensor data for vehicle passing scenario.

\subsubsection{Statistical Sensor Modeling (Individual Sensors)}

The Baysian filter requires specific parameters for both the prediction and correction steps. The following section describes the prediction parameters and correction parameters used for the ultrasonic sensor and magnetic sensor. Data was collected for a vehicle passing scenario as mentioned in the introduction. The methods described in the following sections may be extended to create other prediction and correction steps for scenarios other than the passing scenario that is modeled in the following sections.

\section{Ultrasonic Sensor}

The ultrasonic sensor is used to detect the range and presence of an object on the lateral side of a large truck. The prediction and correction models for this sensor can be achieved because this 
sensor is operating in a specific manner with physical constraints (i.e. lane dimension and vehicle dimensions) and detection frequency (i.e. traffic flow). For this sensor, the state variable of interest is presence of a vehicle. In other words, when the ultrasonic sensor is filtered through the Bayesian filter, the output is the likelihood that a vehicle is present.

To describe the sensors behavior, a perceptual model is required. For this application, the perceptual model was created for the ultrasonic sensor by monitoring the lane on the lateral side of a large truck. As vehicles pass by the sensors, the average distances are recorded and average sensor behavior identified. The sensor behaviors can be modeled as Gaussian distributions and used in the correction step of the filter. The histograms shown below represent the behavior of an ultrasonic sensor when vehicles are present and absent, respectively (Fig. 30 and 31). The average distance of a passing car from this model is about $6.25 \mathrm{ft}$ (sensor voltage of $0.71 \mathrm{~V}$ ). It is important to note that some transmitted signals from the ultrasonic sensor may be reflected off a vehicle's body and not be received by the sensor. This causes the sensor behavior to be somewhat bimodal. The information from this belief distribution is utilized in the prediction step to account for this sensor characteristic.

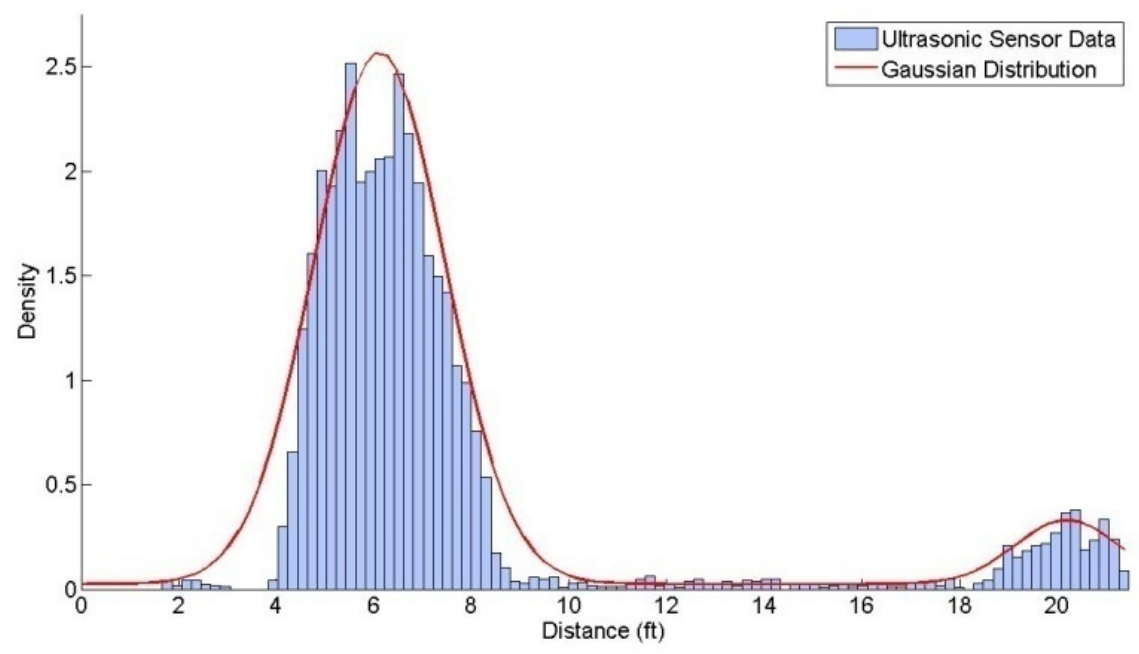

Figure 30. Ultrasonic sensor belief distribution when vehicle present. 


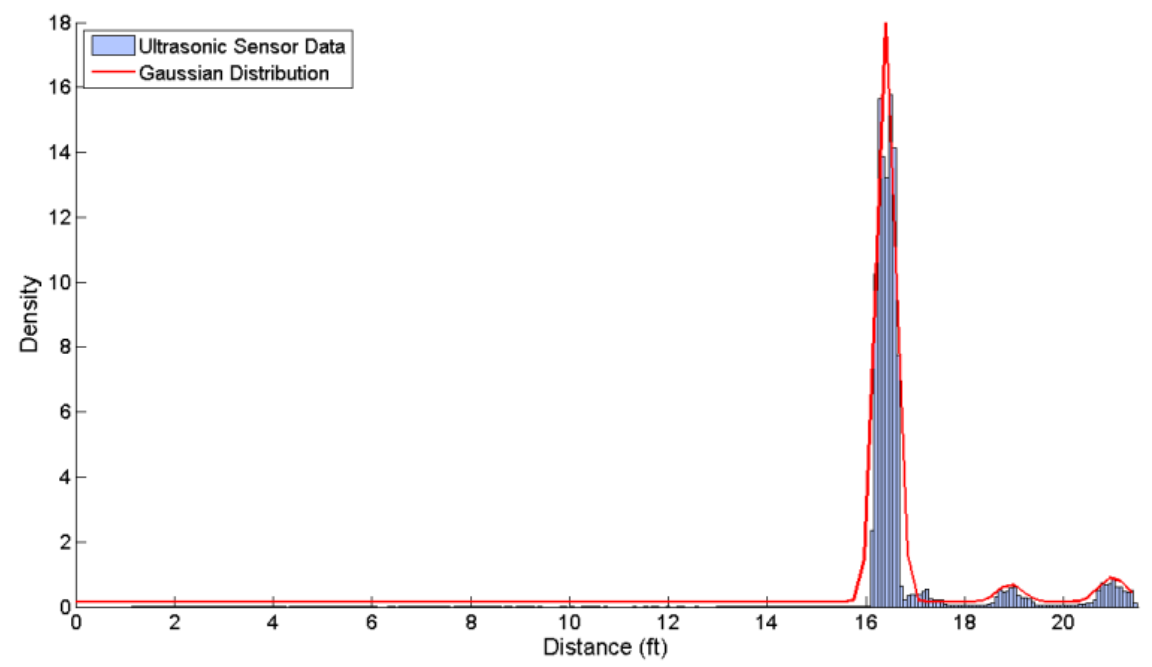

Figure 31. Ultrasonic sensor belief distribution when vehicle absent.

\section{Magnetic Sensor}

The magnetic sensor was tested in the same manner as the ultrasonic sensor. The magnetic fields in all three axes $(x, y, z)$ were recorded as vehicles passed by the sensor. As mentioned in the "Introduction" section, a math function was used to positively detect vehicle presence.

$$
\left|B_{x}\right|+\left|B_{y}\right|+\left|B_{z}\right|
$$

Unlike the ultrasonic sensor when a vehicle passes by a magnetic sensor, the change in sensor voltage is gradual and is not proportional to the vehicle's length. To accurately identify a vehicle's presence from the magnetic sensor, its behavior is modeled as (46) for an incremented range of data rather than frequency.

$$
\frac{\text { Number of "Present" Measurements }}{\text { Total Number of Measurements }}
$$

The behavior for the magnetic data is best described using a two step function and a Gaussian distribution (Fig. 32). The two step function best describe belief distribution of the magnetic sensor behavior in the lower magnetic field ranges and the Gaussian curves best describes the higher field strengths. The same process is followed to describe the magnetic sensor's behavior during the absence of a vehicle. 


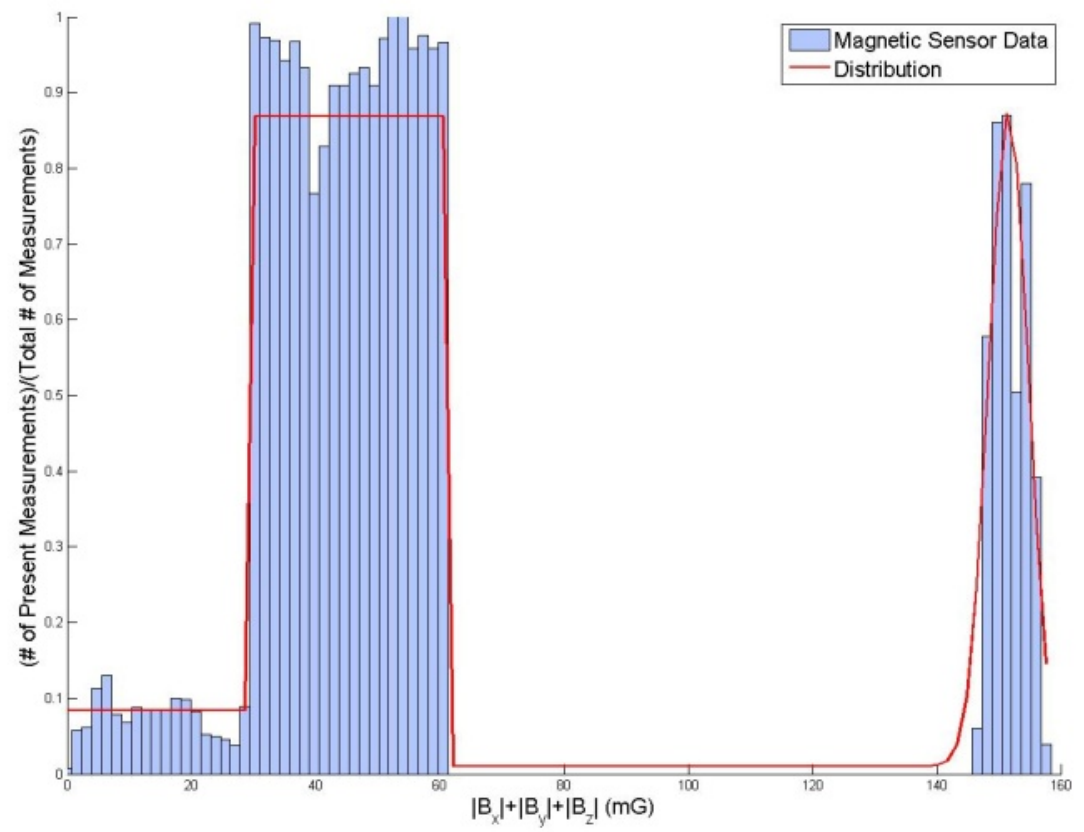

Figure 32. Magnetic sensor belief distribution when vehicle present.

\section{Prediction Model}

The prediction step requires the probability that an event will occur at time $t$ based on the previous correction step at time $t-1$. These parameters can be based on a variety of ideas. In the case of predicting the likelihood of a vehicle being present, two prediction schemes are taken into consideration. One prediction phase is in effect when a vehicle is detected and the other prediction phase is in between vehicles. The first prediction step takes into account the number of "present" measurements taken by the ultrasonic sensor when a vehicle passes; the number of "present" samples varies with the physical length of a vehicle and its relative velocity. As the number of "present" measurements increases, the predicted probability of a vehicle being present in the next sensor measurement will decrease. This process can be further refined by integrating the magnetic sensor to identify the vehicle type allowing adjustments in the number of predicted "present" measurements based on vehicle length. The same approach mentioned is used for the prediction parameter when a vehicle is absent; however, the number of "absent" measurements is based off of vehicle frequency or traffic flow information provided from intelligent transportation 
systems. As traffic flow increases, the likelihood of a vehicle being absent in the next "absent" measurement will decrease. Figure 33 shows the ultrasonic sensor measurement and predicted likelihood when a vehicle is present and absent.
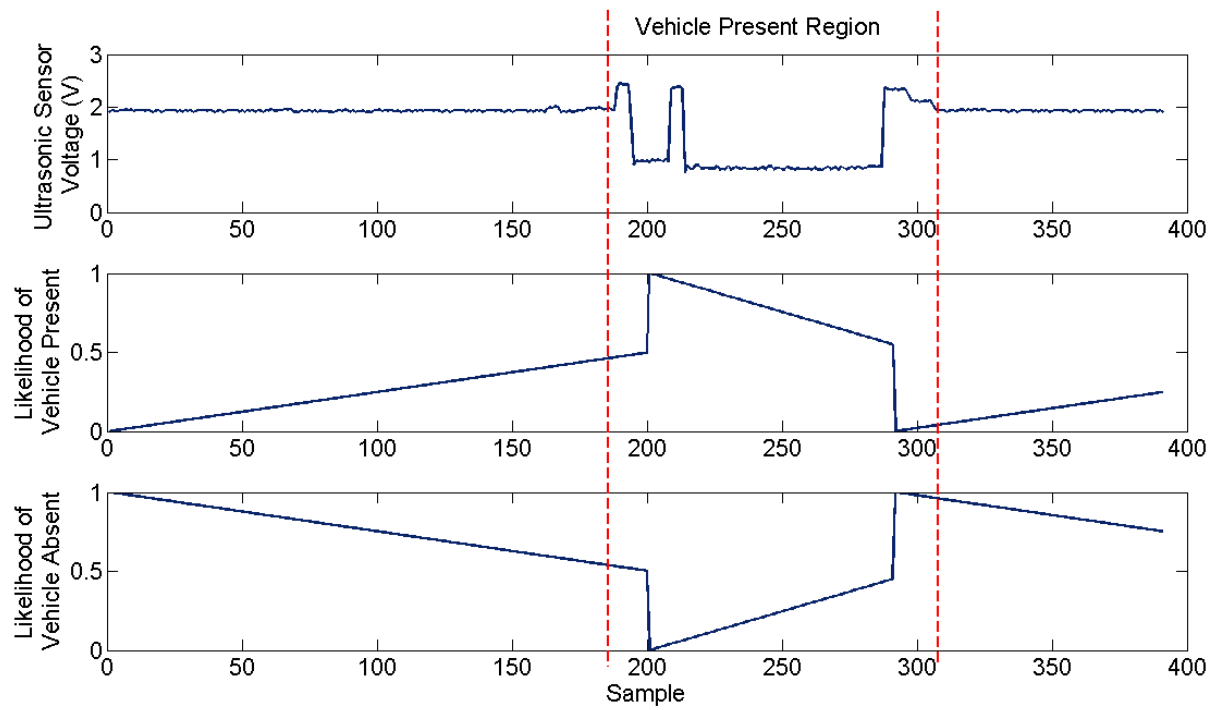

Figure 33. Ultrasonic sensor raw data and predictive models.

\section{Correction Model}

The correction step uses the sensor models to make correction in the predicted measurements. With the ultrasonic and magnetic sensor models and a prediction model, the probability of an object being present and being of specific type can now be identified using equations (42)-(44). The results for this prediction and correction methods being applied to both individual sensor case and sensor fusion case are presented in the next section.

\subsubsection{Bayesian Filter Results (Individual Sensors)}

The Bayesian filter is complete with both the prediction and correction models produced above as shown in the following schematic (Fig. 34). The following shows the resulting behavior of the Bayesian filters for the ultrasonic sensor and magnetic sensor. 


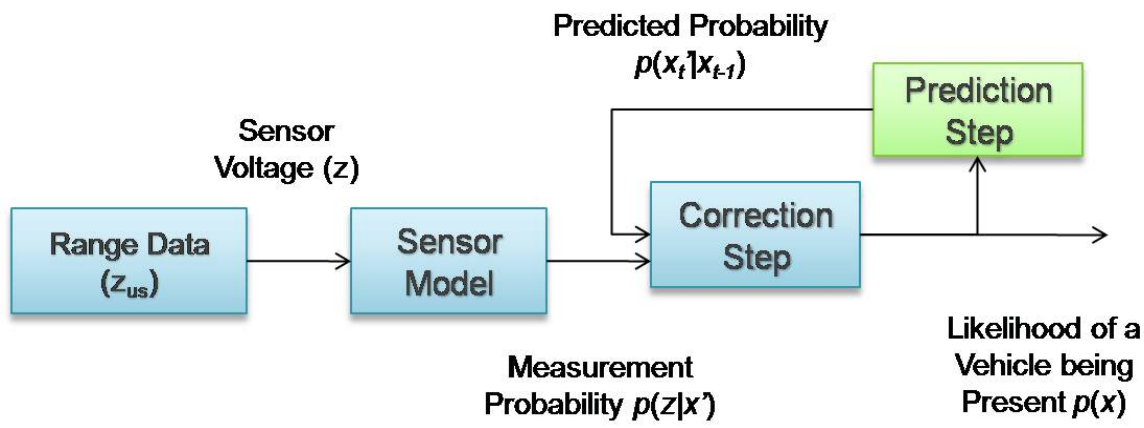

Figure 34. Bayesian filter algorithm (individual sensor).

As vehicles pass the ultrasonic sensor, the data is recorded and entered into the Bayesian filter algorithm (Fig. 34). The prediction model and the correction model, in this algorithm, work together to output the likelihood that a vehicle is present. It can be seen in Fig. 35 that the noise in the ultrasonic sensor, such as the large spike at time step 220 has little effect on the belief that a vehicle is present. The same procedure is used for the magnetic sensor (Fig. 36). With this Bayesian filter, the uncertainties that arise from partial and noisy ultrasonic data are accounted for and the belief of a vehicle's presence can be evaluated to make decisions in vehicle identification.

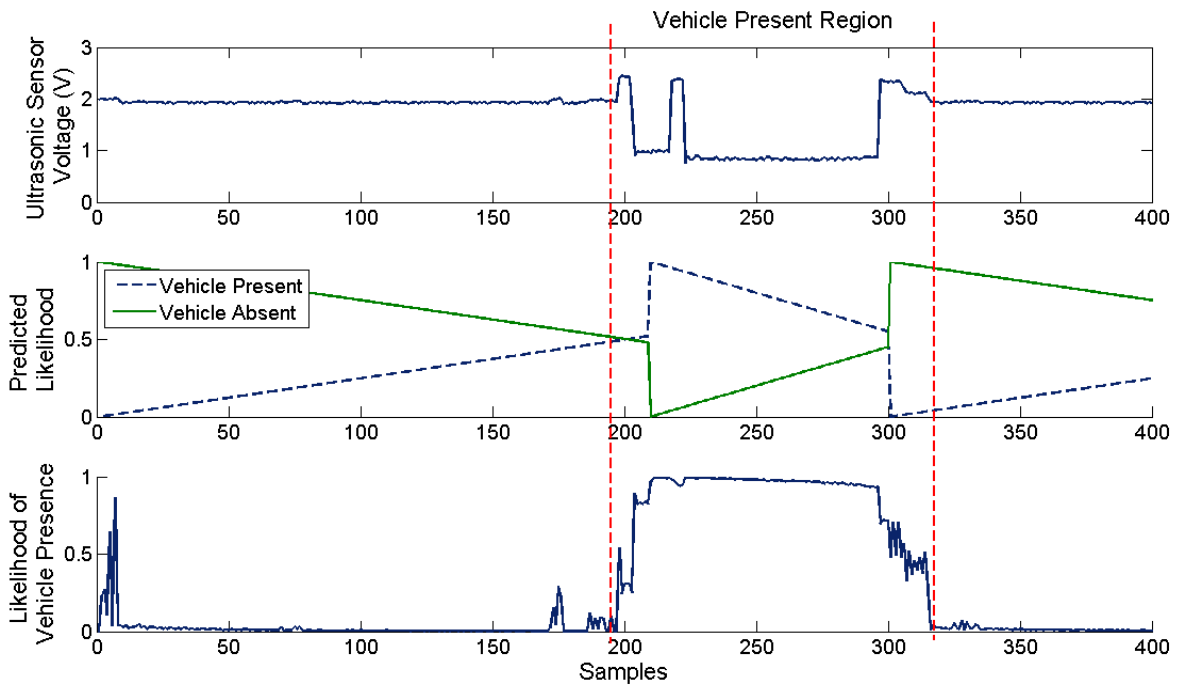

Figure 35. Likelihood of vehicle presence (ultrasonic sensor). 

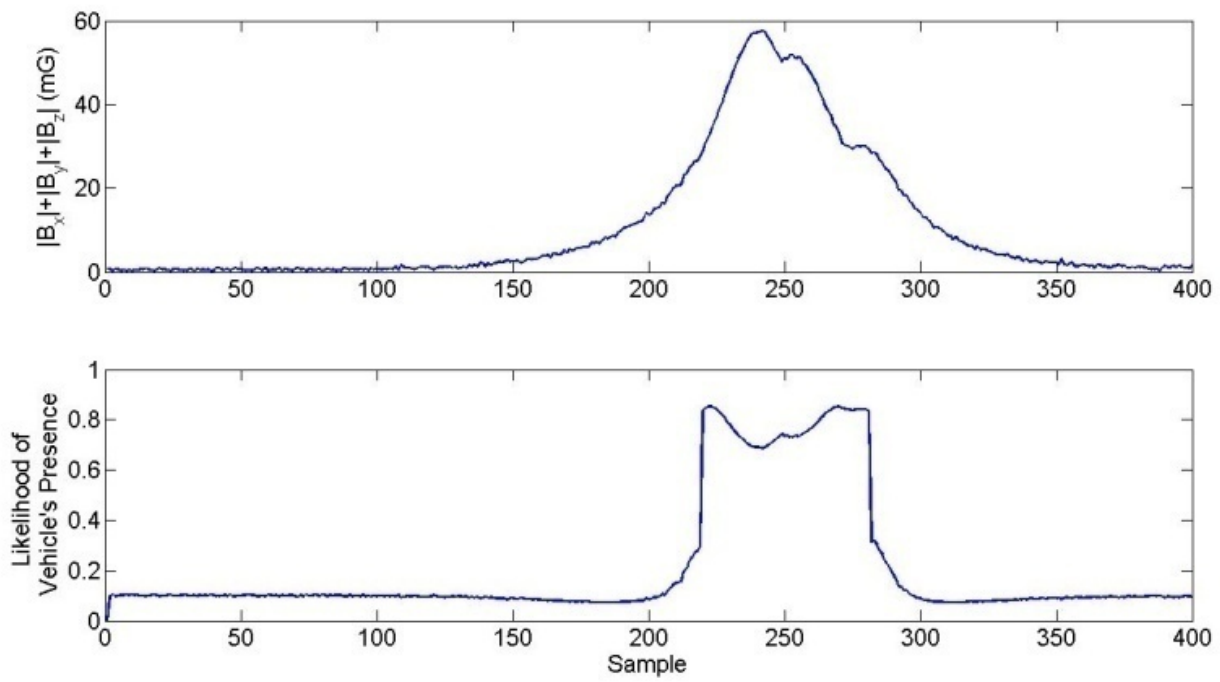

Figure 36. Likelihood of vehicle presence (magnetic sensor).

\subsubsection{Sensor Fusion}

The outputs from the Bayesian filter only represent the belief of individual sensors. Thus, a joint probabilistic method is required to "fuse" this information together (Fig. 37). If the ultrasonic sensor is represented as $S_{l}$ and the magnetic sensor as $S_{2}$, the joint belief distribution can be represented as [21]:

$$
p\left(x \mid z_{S 1}, z_{S 2}\right)=p\left(x \mid z_{S 1}\right) p\left(x \mid z_{S 2}\right)
$$

where, $x$ and $y$ represent independent state variables for the sensors and $z$ represents the joint distribution.

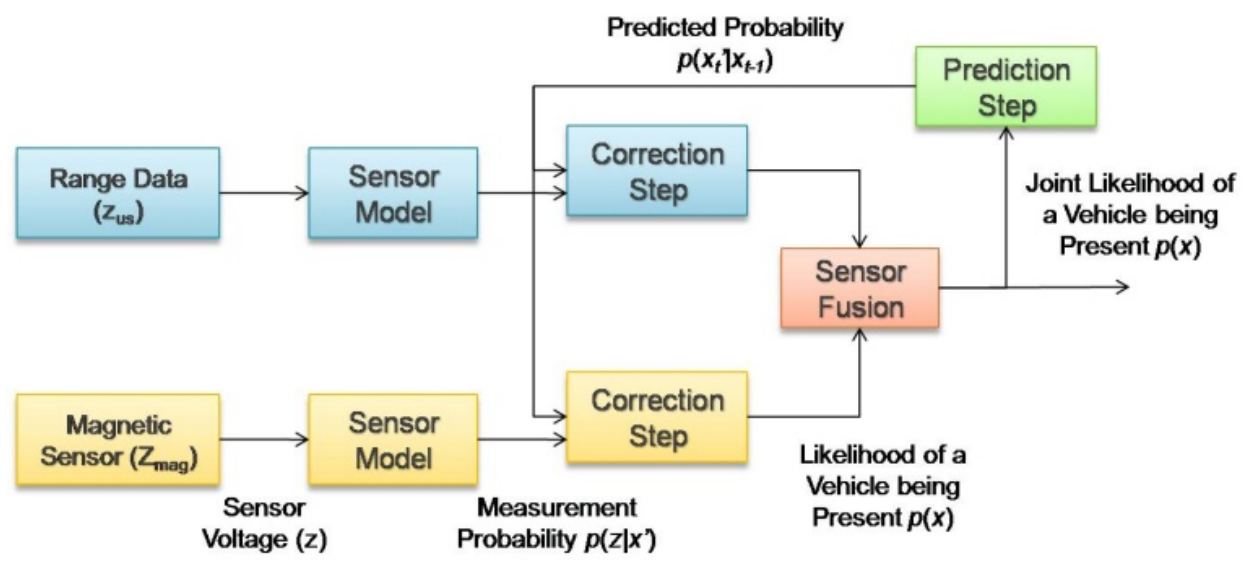

Figure 37. Bayesian filter algorithm (multiple sensors). 

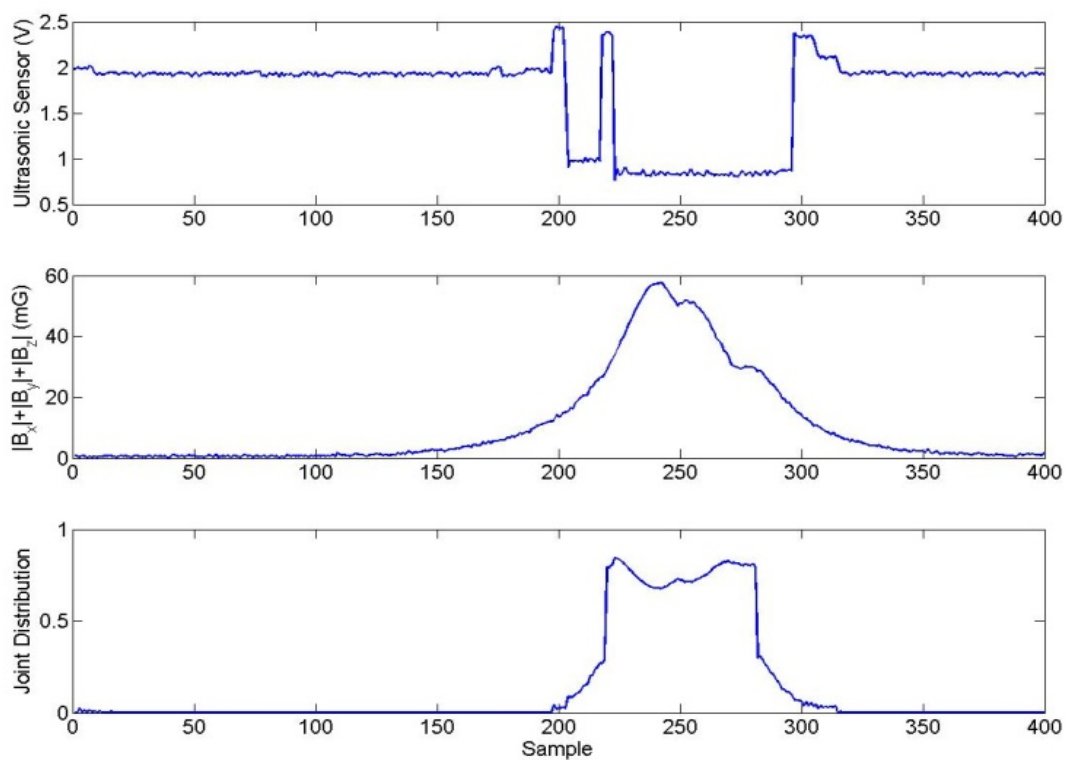

Figure 38. Joint likelihood of vehicle presence.
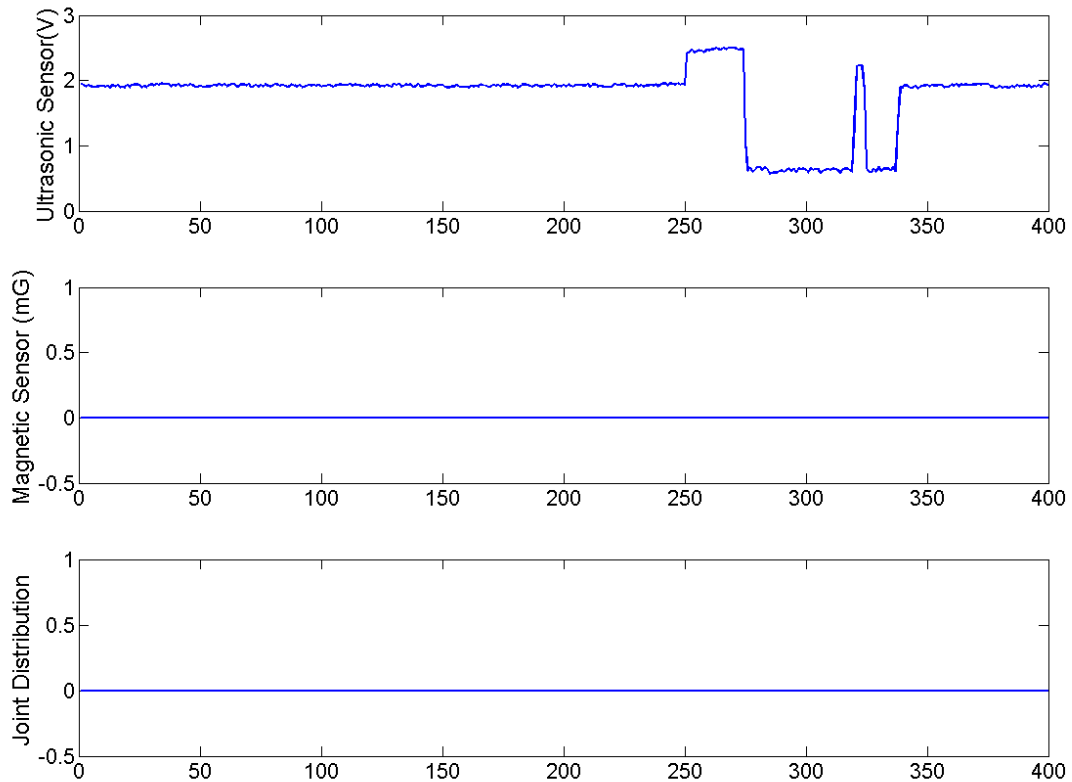

Figure 39. Joint likelihood of a non-metallic object.

Figures 38 and 39 show the detection of two objects; in Fig. 38 the joint likelihood being high (about 0.8 ) suggests the presence of a vehicle and on the other hand, Fig. 39 has a zero joint likelihood suggesting the presence of a non-metallic object. This prediction is justified by observing that the magnetic sensor data is low while only the ultrasonic picks up the presence of an object. 


\subsubsection{Full Scale Testing}

To proficiently test the vehicle detection algorithm described in the previous section, data collection was extended to a class 8 vehicle and collected during normal operation of the vehicle. A test fixture was fabricated to attach the sensors and data acquisition system to a class 8 trailer without disturbing normal operation. The design of this test fixture takes into account: powering the system, protecting the system from excessive vibration, protecting the system from roadway debris, providing the system with proper cooling and ventilation, maintaining accessibility to all components, and attaching the fixture to the exterior of the trailer.

The final test fixture is shown in Fig. 40. The data acquisition hardware required includes a 16 channel data acquisition system for the ultrasonic and magnetic sensors, CAN bus system for radar sensors, and FireWire for the reference camera. The system also includes a $12 \mathrm{~V}$ deep cycle battery and a $120 \mathrm{~V}$ pure sin wave inverter to make the system self-powered. This helps to avoid introducing any noise from the truck's power supply. All hardware in the test fixture are secured or isolated from vibration to avoid any damage that may be incurred from the trailer's vibration. To protect the computer used for data acquisition from excessive vibration, a solid state hard drive is used and the computer is surrounded by packaging foam. To protect the system from roadway debris the text fixture is enclosed in $5 / 8$ " wood and secured to a metal frame with fasteners. The rear panels were fitted with cooling vents to provide ventilation for the computer and the power inverter. All components of the test fixture are accessible through removable rear and top panels. 


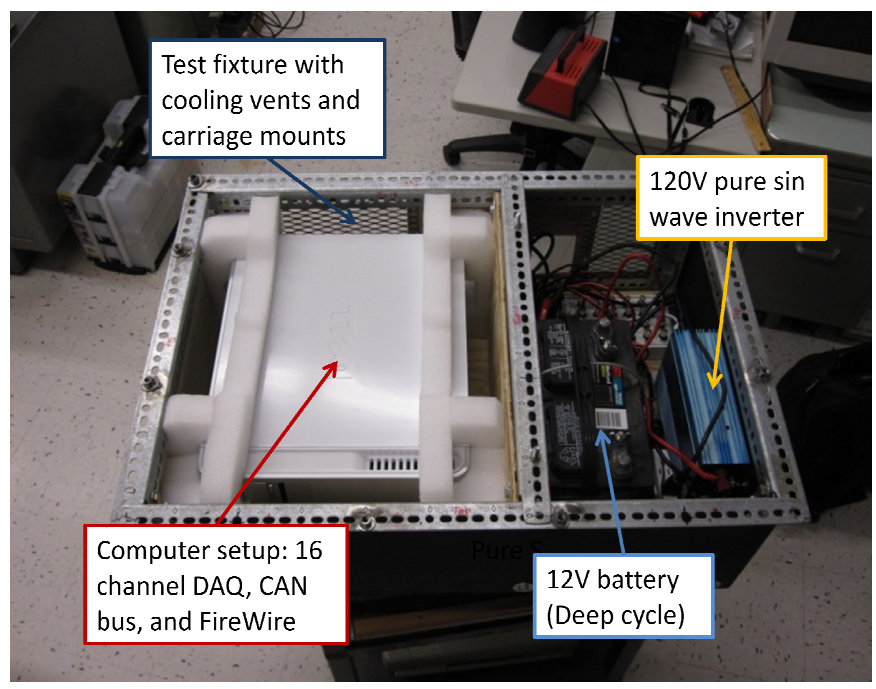

Figure 40. Equipment housing for road testing.

This fixture was designed to attach underneath the trailer to allow for normal use of the trailer.

The test fixture is attached to the trailer using a carriage that is secured to the trailer using I-beam clamps (Fig. 41). The test fixture is slid into the carriage and is secured using fasteners (Fig. 42).

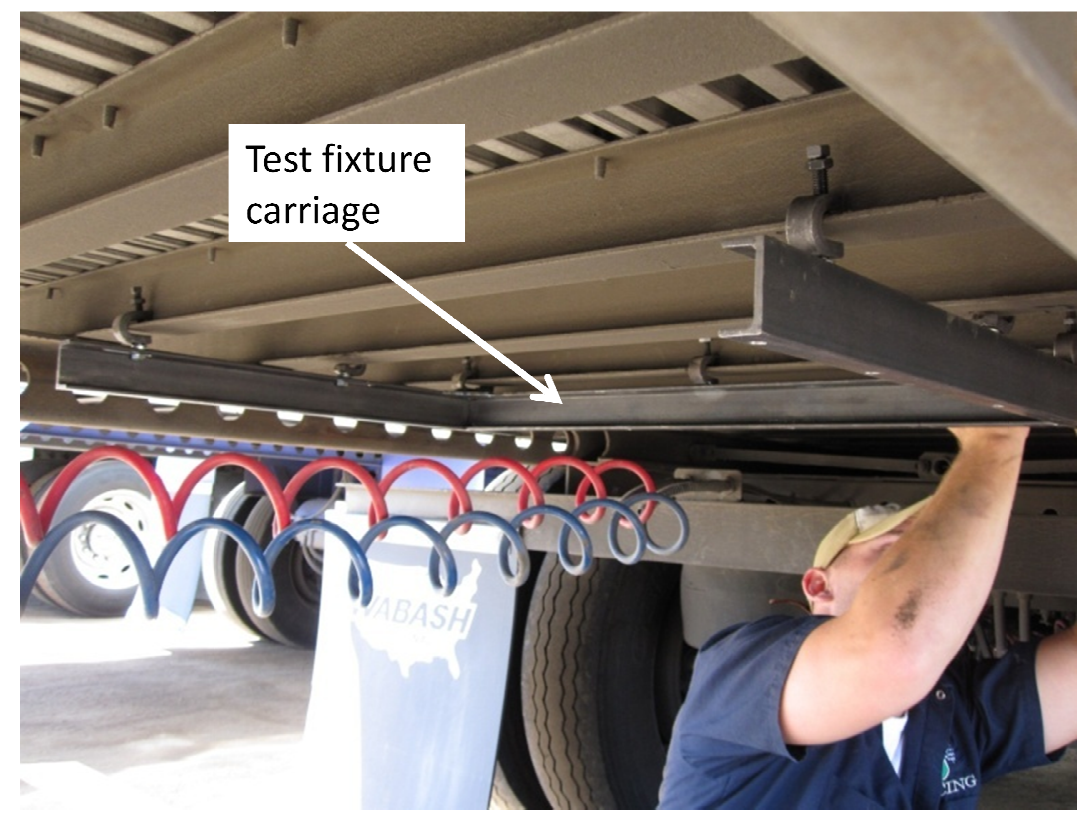

Figure 41. Carriage for equipment housing. 


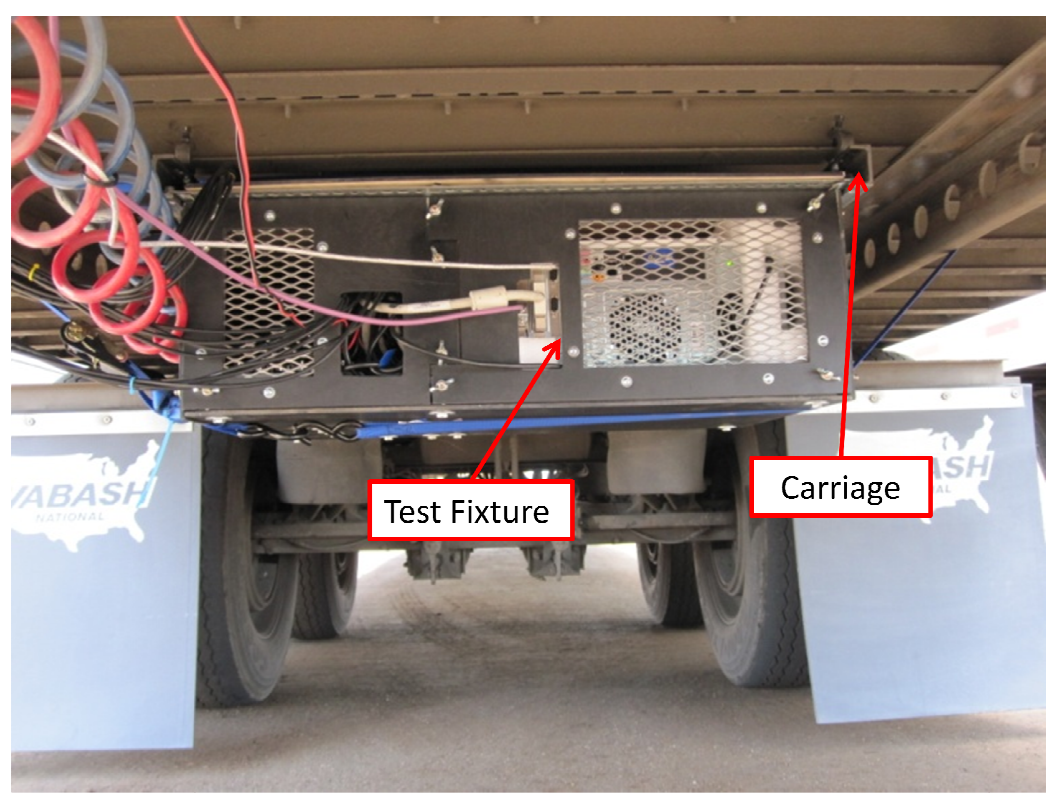

Figure 42. Equipment housing and carriage on large truck.

The ultrasonic sensors, magnetic sensors, and rear facing radar were attached on the left and rear of the trailer (Fig. 43). A combination of one ultrasonic sensor and one magnetic sensor was placed at the rear corner of the trailer and another 6 feet ahead.

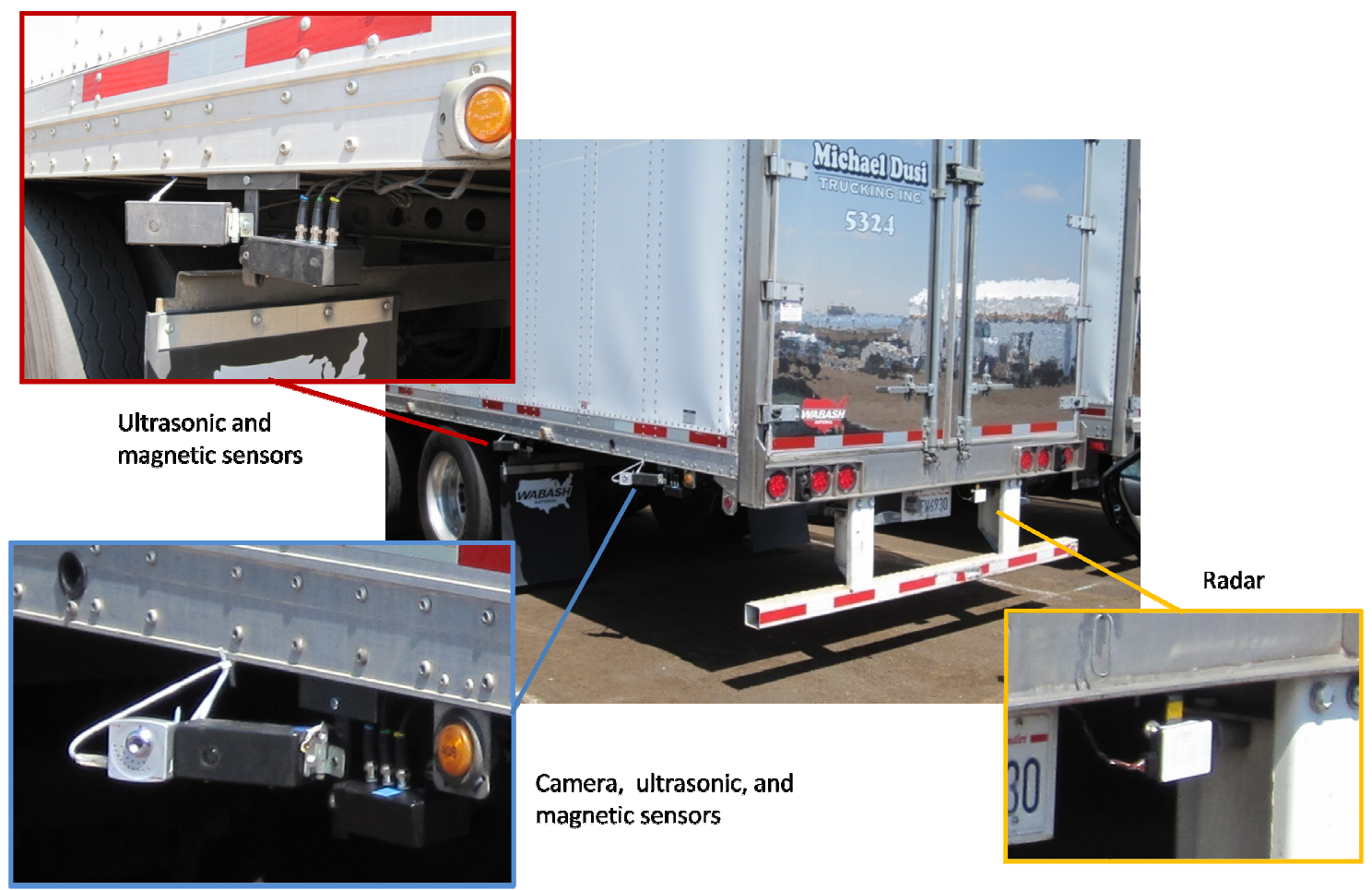

Figure 43. Sensor placement on large truck. 
The Class 8 truck was taken out on its normal delivery route and through several specific passing scenarios. After completion of the testing it was discovered that the ultrasonic sensor data was noisy due to an electrical grounding issue and the data from both ultrasonic sensors could not be used. The results indicated that the magnetic and radar sensors functioned as expected. Video was also acquired for reference.

Due to time constraints, additional testing on the class 8 truck could not be conducted for this study. The final tests were conducted using a pickup truck driven through various passing scenarios. The data from these tests is free from the induced noise of the full scale test and is comparable to the conditions of the full scale tests. The data analysis presented in the following section uses this data. 


\subsection{DATA ANALYSIS}

Several road tests were carried out to test the performance of the Bayesian filter algorithm in the detection of vehicles and the rejection of false targets. The tests conducted assess the performance of the filter while passing a variety of targets that could affect the sensors, such as non-vehicle magnetic objects and objects with dimensions similar to vehicles. The following sections include: how the filter performance is quantified, the filter performance for different passing scenarios, and recommendations for future work to improve the filter's performance. The following sections include: Quantification of filter performance, filter performance for different passing scenarios, and recommendations for future work to improve the filter's performance.

\subsubsection{Bayes Filter Algorithm Performance}

The performance of the Bayes filter is quantified using the percentages of true and false outputs of the filter. Using the camera data as a reference of true vehicle presence and the vehicle presence likelihood output of the filter, the number of true and false filter outputs can be calculated. Figure 44 shows the four possible outputs of the filter. The definitions and case names that will be used for the remainder of this paper are described in Table 2. For this study, an output from the filter is considered true (Case 1 and 2) if a vehicle is present (determined from the video data) and the filter likelihood is over 0.5 (Case 1) or if a vehicle is not present and the filter likelihood is under 0.5 (Case 2). The filter output is considered false (Case 3 and 4) if a vehicle is present and the filter likelihood is under 0.5 (Case 3) or if a vehicle is not present and the filter likelihood is over 0.5 (Case 4). 


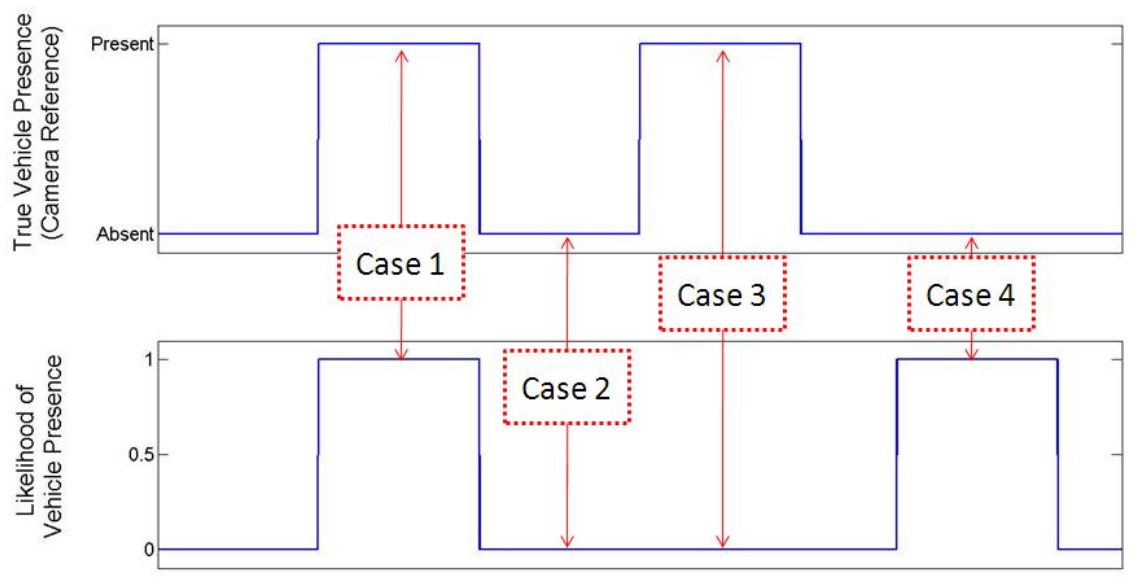

Figure 44. Filter performance.

TABLE 2. Filter performance cases.

\begin{tabular}{|c|c|c|c|c|}
\hline Case & $\begin{array}{c}\text { Filter } \\
\text { Output }\end{array}$ & Case Name & Description & Action Taken \\
\hline 1 & \multirow{2}{*}{ True } & True Present & Filter reports a vehicle accurately & $\begin{array}{c}\text { Proper action or } \\
\text { warning taken }\end{array}$ \\
\cline { 3 - 5 } 2 & True Absent & $\begin{array}{r}\text { Filter reports a "non-vehicle" target } \\
\text { accurately }\end{array}$ & No action taken \\
\hline 3 & \multirow{2}{*}{ False } & False Present & $\begin{array}{c}\text { Filter reports a "non-vehicle" target } \\
\text { when a vehicle is present }\end{array}$ & $\begin{array}{c}\text { Required action or } \\
\text { warning not taken }\end{array}$ \\
\cline { 3 - 5 } & False Absent & $\begin{array}{r}\text { Filter reports a vehicle when no } \\
\text { vehicle is present }\end{array}$ & $\begin{array}{c}\text { Over-correction or } \\
\text { false warning }\end{array}$ \\
\hline
\end{tabular}

The performance of the Bayes filter can now be calculated by classifying the filter outputs. Three types of percentages are used to describe the performance of the filter: the overall performance, the vehicle present percentage, and the vehicle absent percentage (Table 3). The overall performance is the percentage of how many true filter outputs (Case 1 and 2) were made over the entire data set. The vehicle present performance percentage shows how many true present outputs (Case 1) were made for the total number of data points where vehicles were present. The vehicle absent performance percentage shows how many true absent outputs (Case 2) were made for the data points where vehicles were not present. The overall performance shows how many correct 
outputs the filter can produce while the vehicle present and vehicle absent performances show whether the filter is biased to positive or negative detections of vehicles.

TABLE 3. Performance percentages.

\begin{tabular}{|c|c|}
\hline Performance (\%) & Equation \\
\hline Overall & $\frac{\text { (True Present }+ \text { True Absent })}{\text { Total Number of Data Points }} \times 100 \%$ \\
\hline Vehicle Present & $\frac{\text { True Present }}{\text { Total Number of Present Points }} \times 100 \%$ \\
\hline Vehicle Absent & $\frac{\text { True Absent }}{\text { Total Number of Absent Points }} \times 100 \%$ \\
\hline
\end{tabular}

Analyzing the Bayes filter performance also requires considerations of the testing environment. The sensor models created in Section 3.2.2. were created in a controlled environment where vehicle data was collected when the sensors are stationary. During the road testing, it was apparent that sensor environment has more variance and is more volatile than the environment where data was collected in Section 3.2.2.To improve the implementation of this filter in this unstable environment, further testing and data collection are required to include the sensor variations into the statistical sensor model. Other considerations for improving the filter performance are addressed in Section 3.3.3.

To demonstrate the effects of the sensor behavior on the filter performance, the results presented in the following section show the behavior of the Bayes filter algorithm using two independent methods: first, with the developed sensor model created in Section 3.2.2. and second, using a tuned sensor model. The tuned sensor model is created by modifying the ultrasonic sensor variance and the expected magnetic field ranges of the developed sensor model. The tuned sensor model optimizes the performance of the Bayes filter algorithm in each of the individual scenarios presented in the following section. The results for each individual scenario are presented in the format of Table 4. This data can be interpreted as a best and worst case performance measure. 
This table presents the overall, vehicle present, and vehicle absent performance percentages for both the developed and tuned sensor models.

TABLE 4. Example performance results table.

\begin{tabular}{|c|c|c|c|}
\cline { 2 - 4 } \multicolumn{1}{c|}{} & Overall & Vehicle Present & Vehicle Absent \\
\hline Developed Model & $--\%$ & $--\%$ & $--\%$ \\
\hline Tuned Model & $--\%$ & $--\%$ & $--\%$ \\
\hline
\end{tabular}

\subsubsection{Filter Performance}

The following section shows the performance of the filter algorithm and the percentage of true and false detection when the host vehicle passes various types of targets. In many cases, it is possible for non-vehicular objects to have the same sensor behavior that a vehicle may have. For example, foliage on the side of the road may cause the ultrasonic sensor to output a high belief that a vehicle is present or railroad track may cause the magnetic sensor to output a high belief that a vehicle is present. The tests conducted show how the individual sensor behavior affects the overall belief of a vehicle's presence. The following scenarios were selected to test the filter's ability to discriminate from true vehicle detection and false targets. The targets include:

- Two passenger vehicles - True vehicle detection with high belief of vehicle presence for both the ultrasonic sensor and magnetic sensor.

- Set of garbage containers - Possible false warning with high belief of vehicle presence for ultrasonic sensor and low belief for magnetic sensor.

- Railroad tracks - Possible false warning with low belief of vehicle presence for ultrasonic sensor and high belief for magnetic sensor.

- Building wall - Possible false warning with high belief of vehicle presence for both the ultrasonic sensor and magnetic sensor.

Most of the above tests are less than 10 seconds in length; however, they represent the typical behavior of the Bayes filter algorithm during regular operation. 
Two sensors models are presented in each of the case scenarios: a developed model and a tuned model. The developed model represents the sensor models developed in Section 3.2.2. The sensor models for the ultrasonic and the magnetic sensor were created in controlled environment; but as expected, the variance in the sensors' behavior during the road test is not fully captured in the developed model. The performance of the Bayesian filter using the developed model is presented, but does not represent the full capability of the filter. To demonstrate the maximum performance of the filter during road testing, the developed model was tuned by adjusting the expected values for the ultrasonic sensor and the magnetic sensor to values that maximize the filter performance. It was found that the expected value and variance of the ultrasonic sensor of the developed model provide suitable filter performance. However, the expected value for the magnetic sensor was changed from a range of $45 \mathrm{mG}$ to $20 \mathrm{mG}$ to maximize filter performance. This change was made to fit the actual road test data, which better represents typical driving conditions. The tuned model was implemented to also demonstrate that the filter performance can be further increased by creating more advance sensor models that can account for the sensor behavior variance that exist in the real world.

\section{Two Passenger Vehicles}

In this scenario, the host vehicle passes two passenger vehicles in quick succession. This scenario tests the baseline performance of the Bayes filter. It can be seen in Fig. 45 that the ultrasonic sensor voltage goes low and the magnetic sensor voltage goes up when a vehicle is present (34.5$35.5 \mathrm{~s}$ and 35.7-36.6 s). The developed sensor model does not report the presence of the first vehicle because the magnetic field amplitude is expected to be between 29 and $62 \mathrm{mG}$ (Fig. 32). With the developed model the overall performance is $70.6 \%$. For maximum performance, the tuned sensor model shifts the expected magnetic field amplitude to the range of 10-30 mG. This brings the overall sensor performance to $90 \%$. 


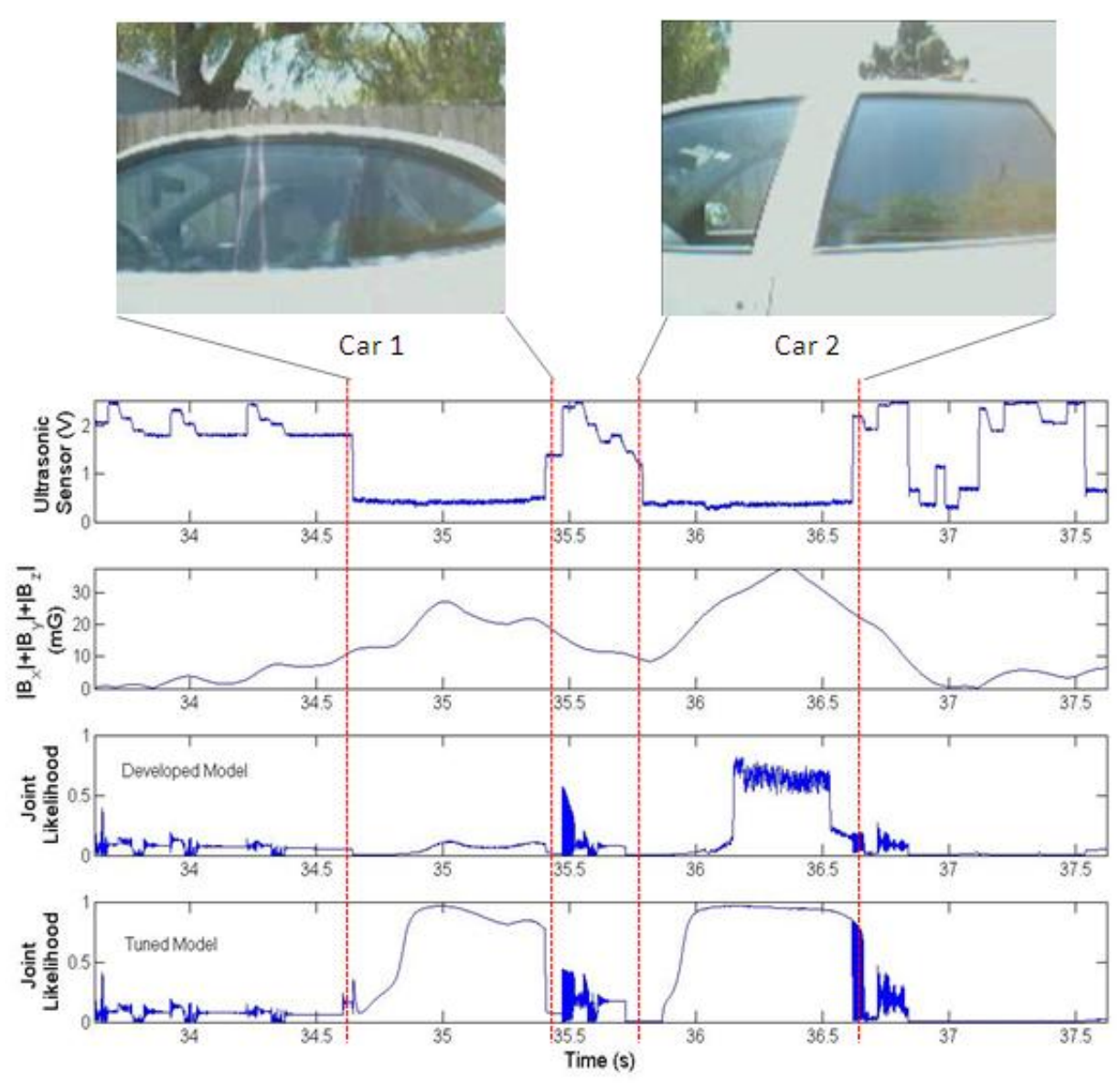

Figure 45. Two passenger vehicles test using developed and tuned senor models.

It can be seen in Table 5 that the filter is biased toward Vehicle Absent; meaning that the filter is more likely to report that a vehicle is not present. This has an advantage of not setting off false alarms, but also indicates that further testing is necessary for irreversible decision making.

TABLE 5. Filter performance while passing two passenger vehicles.

\begin{tabular}{|c|c|c|c|}
\cline { 2 - 4 } \multicolumn{1}{c|}{} & Overall & Vehicle Present & Vehicle Absent \\
\hline Developed Model & $70.6 \%$ & $26.4 \%$ & $100.0 \%$ \\
\hline Tuned Model & $90.7 \%$ & $78.4 \%$ & $99.1 \%$ \\
\hline
\end{tabular}

\section{Passenger Vehicle and Set of Garbage Containers}

This scenario has the host vehicle passing both a vehicle as well as a set of trash containers. The trash containers produce an ultrasonic signature that is similar to vehicles. As the host vehicle passes the target the Bayes filter is able to distinguish between the vehicle and the trash bins. In 
Fig. 46, the ultrasonic sensor voltage goes low for both the passenger vehicle and the trash containers; however, the magnetic sensor voltage only changes for the vehicle. It can be seen when using the developed model that the vehicle has a high probability of being present as opposed to the garbage containers. The same tuned model (with the reduced range of magnetic field strength) is applied to this data.

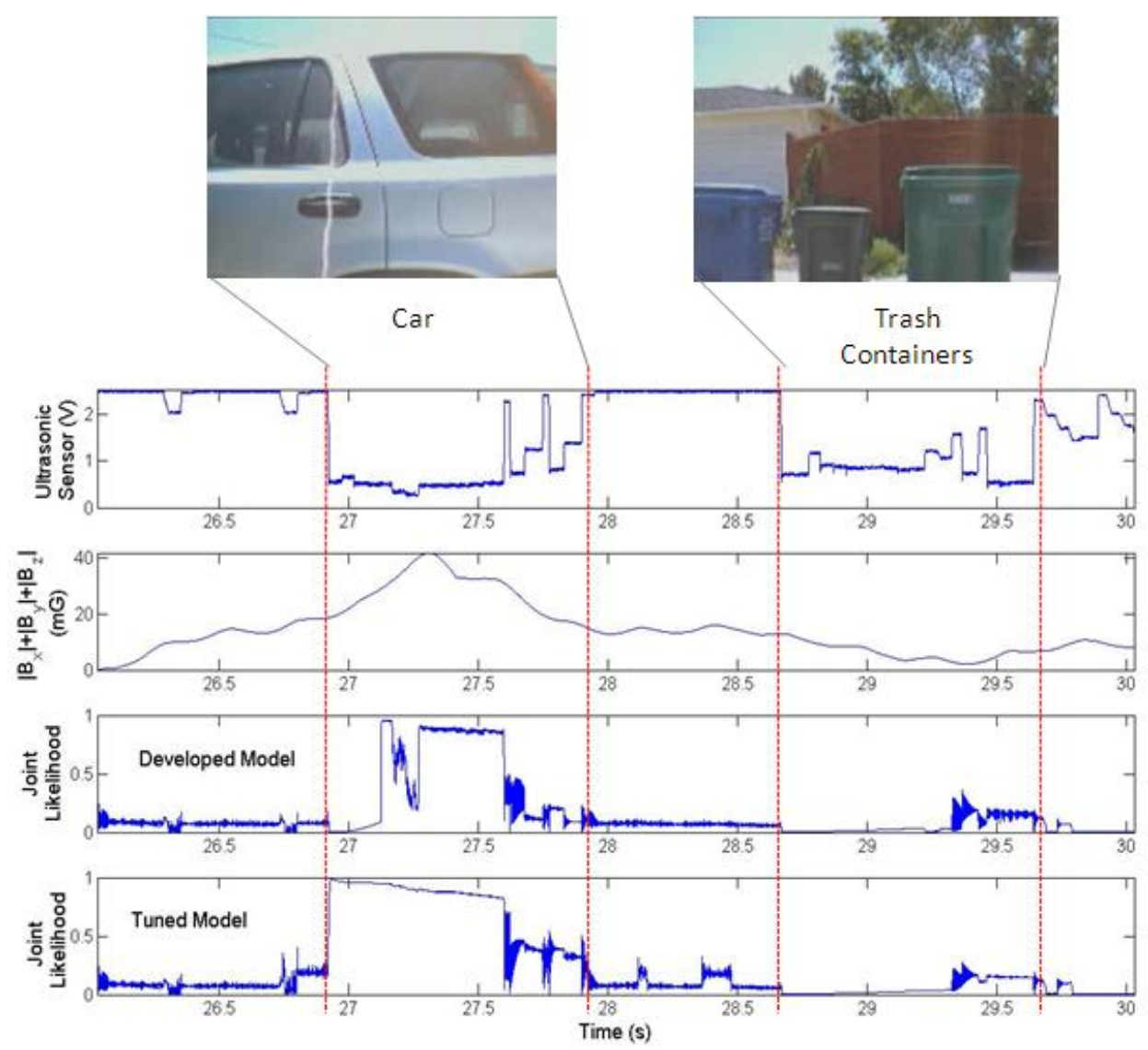

Figure 46. Trash containers test using developed and tuned sensor models.

The filter performs with $91.7 \%$ accuracy with the developed model and $98.2 \%$ with the tuned model (Table 6).This scenario shows that the Bayes filter has the ability to reject non-vehicular objects that have heavy influence on one of the two sensors.

TABLE 6. Filter performance while passing a vehicle and trash containers.

\begin{tabular}{|c|c|c|c|}
\cline { 2 - 4 } \multicolumn{1}{c|}{} & Overall & Vehicle Present & Vehicle Absent \\
\hline Developed Model & $91.7 \%$ & $56.0 \%$ & $100.0 \%$ \\
\hline Tuned Model & $98.2 \%$ & $90.7 \%$ & $99.9 \%$ \\
\hline
\end{tabular}




\section{Railroad Tracks}

The next scenario conducted had the host vehicle drive over railroad tracks. The railroad tracks have a high magnetic influence, but no influence from the ultrasonic sensors. It can be seen that the Bayes filter continues to output a low likelihood of a vehicle's presence when the host vehicle passes over railroad tracks.

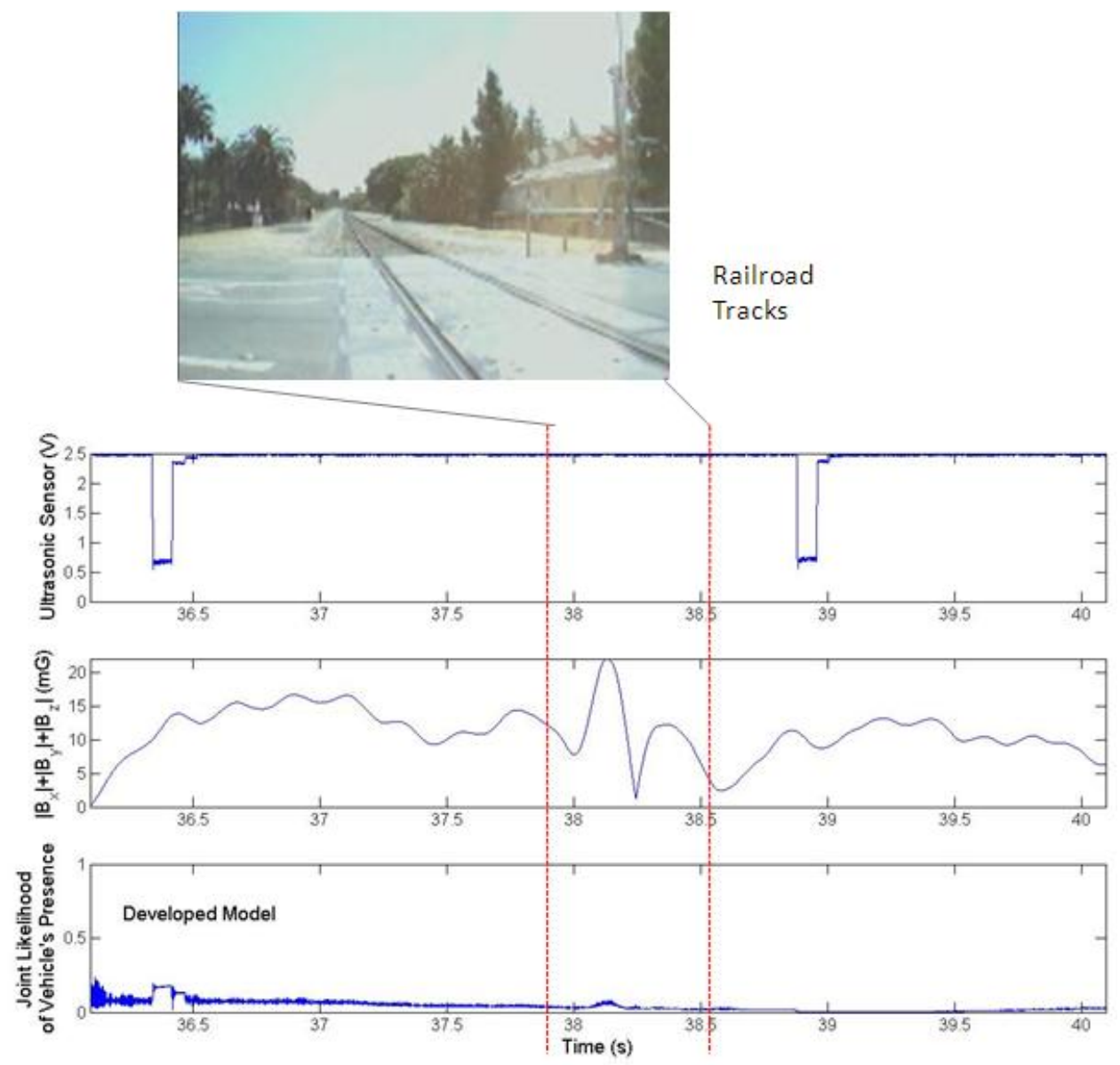

Figure 47. Railroad tracks test using developed sensor model.

In this scenario, the Bayes filter performed at $100 \%$ with the developed model (Table 7). The use of a tuned model was not applied to this scenario because the filter performance could not be further enhanced.

TABLE 7. Filter performance while driving over railroad tracks (high magnetic influence).

\begin{tabular}{|c|c|c|c|}
\cline { 2 - 4 } \multicolumn{1}{c|}{} & Overall & Vehicle Present & Vehicle Absent \\
\hline Developed Model & $100.0 \%$ & N/A & $100.0 \%$ \\
\hline
\end{tabular}




\section{Building Wall}

In this last scenario, the host vehicle is driven past a metal warehouse. The building wall has high influence on both range and magnetic signature. The host vehicle passes the start of the building around $16 \mathrm{~s}$. and passes the end of the building around $26.8 \mathrm{~s}$. In between those times, the ultrasonic sensor voltage is low as if a vehicle was present and the magnetic signature is high as well.

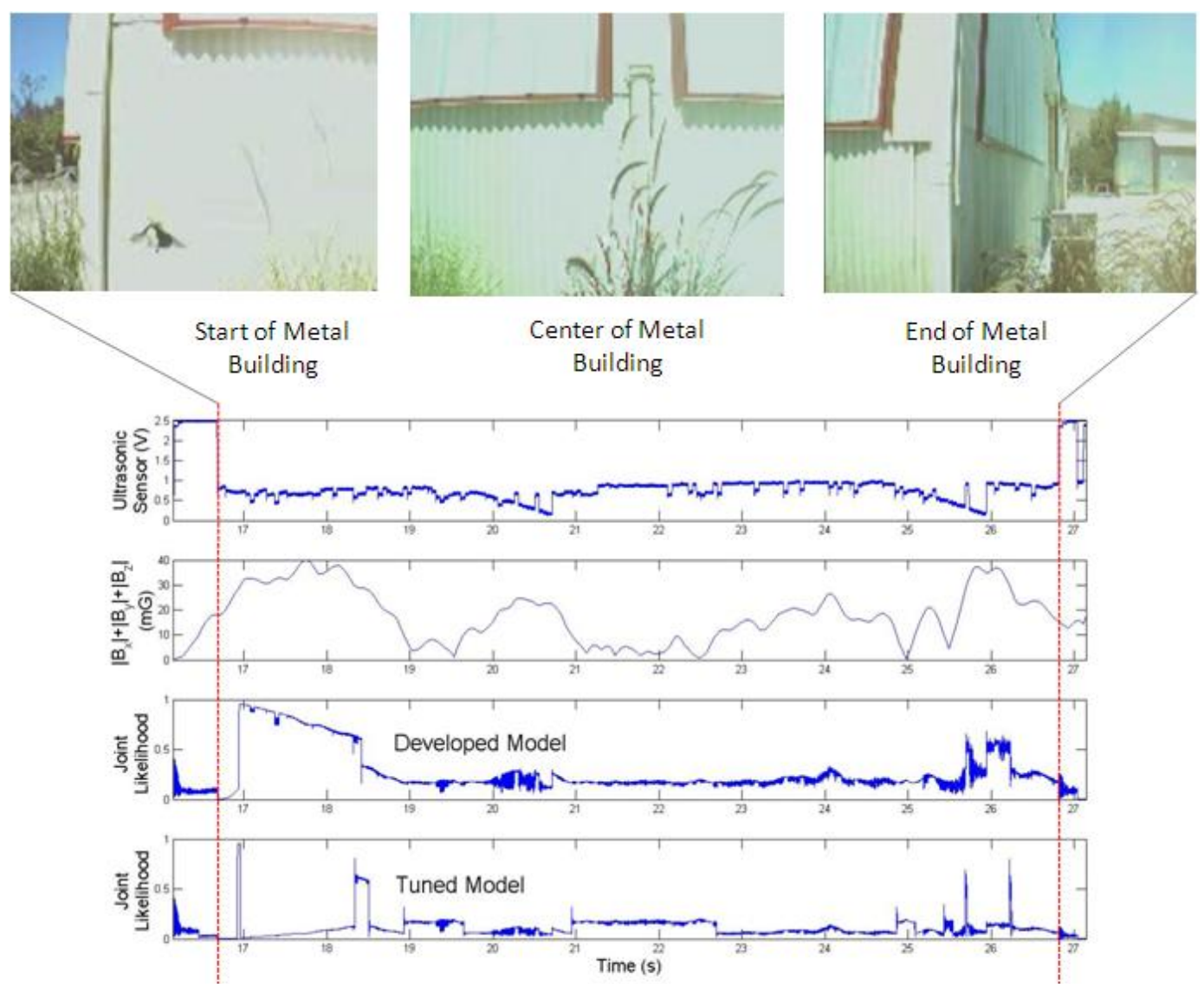

Figure 48. Metal building test using developed and tuned sensor model.

It can be seen in Fig. 48 that when the host vehicle first passes the building the filter has a high belief that a vehicle is present; however, that belief changes at $18.5 \mathrm{~s}$. This is due to the prediction model created in Section 3.2.2. The prediction model (Fig. 49) expects a vehicle to be in the sensor's field of view for $1.5 \mathrm{~s}$. As time increase the likelihood that a vehicle is present is reduced because a vehicle is expected to be within some dimensional constraints. 
Note that in final implementation of this system, the expected duration of vehicle presence could be controlled by host vehicle speed and road type (if available). However, this prediction technique still has an issue if a vehicle pulls alongside a truck and then matches speeds. More work is needed to address that scenario.
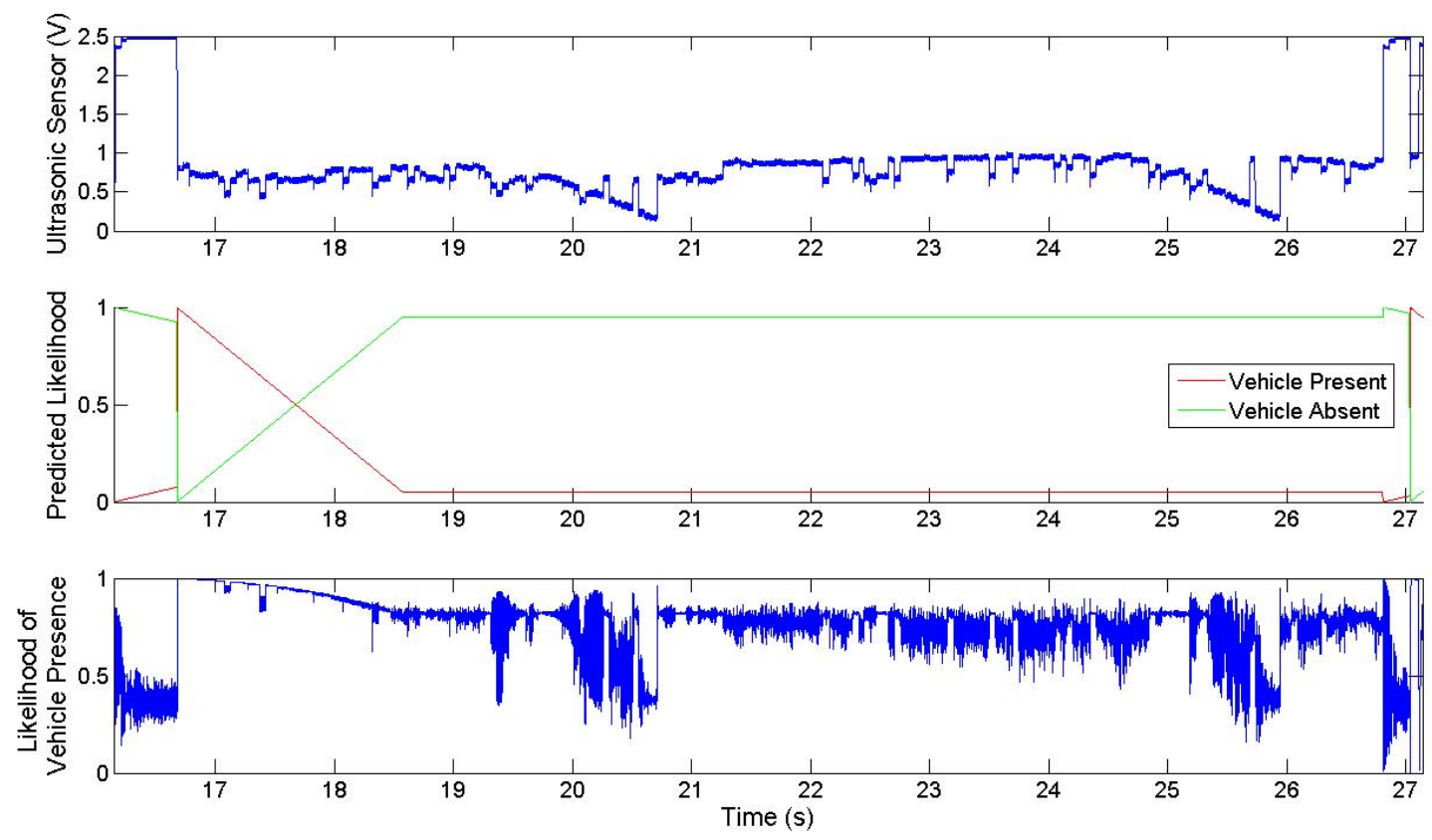

Figure 49. Ultrasonic sensor filter.

In this scenario, the filter had a delayed reaction in identifying that no vehicle was present. The filter has shown the ability to reject false positive targets with both strong range and strong magnetic influence. The overall performance of the filter was $84.2 \%$ (Table 8 ). The same tuned model was applied to further increase the filter's performance to $97.9 \%$.

TABLE 8. Filter performance while driving over railroad tracks (high magnetic signature and high range influence).

\begin{tabular}{|c|c|c|c|}
\cline { 2 - 4 } \multicolumn{1}{c|}{} & Overall & Vehicle Present & Vehicle Absent \\
\hline Developed Model & $84.2 \%$ & N/A & $84.2 \%$ \\
\hline Tuned Model & $97.9 \%$ & N/A & $97.9 \%$ \\
\hline
\end{tabular}




\subsubsection{Improving Filter Performance}

The Bayes filter has room for improvement in two areas: the predictive model and the corrective model. The following sections discuss the specific tasks that have an opportunity to improve the filter's performance and increase the consistency of that performance.

\section{Prediction Model}

Correction model input - The current prediction model is based on edge detection of the ultrasonic sensor, thus is influenced by noise of the sensor. To minimize these noise effects the prediction model must see a consistent low voltage or high voltage (about $300 \mathrm{~ms}$ ) before it can take any action. Using the correction step to input the prediction step instead of using the sensor reading will allow the filter to act without this delay and the effects of the ultrasonic sensor will not affect the prediction step. To modify the current prediction model a study of when the correction step can be triggered to properly identify the presence of a vehicle must be completed. While the current trigger takes a continuous low voltage for a period of $300 \mathrm{~ms}$ to identify the presence of a vehicle, a prediction model based off the correction step may only require one data point above $50 \%$ belief, for example.

\section{Correction Model}

$\underline{\text { Sensor model variance }}$ - The correction model that was used in the study was created from sensor data collection in a single controlled environment which consisted of the host vehicle parked on a roadside while other vehicles passed at low speeds. This data was used to generate the correction model. Vehicle detection was consistently above $80 \%$ accurate when the algorithm was operated under similar conditions. However, when the system was tested under conditions that differed significantly from those used to generate the correction model, the consistency and accuracy was lower. To improve the performance of the filter, sensor data collection should be extended to include more extensive "real-world" scenarios. In the road tests that were conducted the 
ultrasonic sensor variance is much greater than the controlled scenario that the sensor model was based on. In the tuned sensor models shown above the standard deviation of the sensor model was increased to allow the likelihood of the vehicle presence to decrease at a slower rate as the vehicle were detected closer and farther than the expected value of the sensor model. The system could be designed to change from one correction model to another depending on external factors such as location from GPS system, live traffic data, or vehicle-to-vehicle communication. For example one correction model could be used at low speeds on surface roads, a second for low speeds on highways, a third for high speeds on highways, etc.

Weighting individual sensors - Observations were made while comparing the video of vehicles passing to the ultrasonic sensor and magnetic sensor. It was seen that in some cases, the ultrasonic sensor produced a belief that better represented the true vehicle presence than the magnetic sensor and vice a versa. The correction step may be further improved if the algorithm is extended to give an influential weight to the sensor that has a better representation of the true vehicle presence or the higher belief. An influential weight allows the algorithm to internally judge its own belief. An investigation on different statistical methods to change weighting factors is recommended.

Additionally the weighting factors could be changed based on road conditions in a similar manner as the correction model.

\subsection{RESULTS MAPPING}

The following sections present the areas that are most suitable for the implementation of the purposed crash avoidance system. These areas include:

- Improving truck driver awareness

- Improving nearby driver awareness

- Preventing risky actions

- Taking preventative actions 


\subsubsection{Improving Truck Driver Awareness}

A first level of countermeasure would be to use the sensing system to improve the driver's awareness of the truck's surroundings. This could be either continuous (a situation display) or only when objects of interest are detected (a warning system). Information exchange would be visual, audible, or haptic. These safety countermeasures rely on the driver taking the necessary action once alerted to a dangerous situation.

\section{Continuous Situation Display}

Since the system would be constantly scanning the rear and sides of the truck, information on the current status of these scans could be used to provide a continuous update to the driver.

\section{Visual}

Some sort of visual display would be added to the truck to indicate the presence or lack of objects nearby. This could be as simple as a 'green/yellow/red' indicator light showing the presence of any vehicles in the detection range, or as complicated as a full-color display showing the top of the truck and the positions and vehicle types for any objects in the truck's vicinity.

\section{Provide Warnings}

Instead of providing feedback continuously, information from the sensors could be conveyed to the driver only when a vehicle is within the detection range. All driver warning systems carry some risk of precipitating risky actions by startling the driver. Human factors are critical in the selection of an appropriate warning system. 
Visual

A visual warning would be implemented in much the same way as a continual visual display. The only difference is that the driver would only see the warning when objects of interest are present. As a result, the warning could be more intrusive (e.g. heads-up-display, flashing lights).

Audible

Warning sounds could be used to indicate of the presence of nearby objects, as well as their approximate locations (by using the truck's 4-channel speaker system). The main benefits of this option are low cost and less distraction potential.

\section{Haptic}

The steering wheel or seat mounts could be used to give some sense of warning such as a vibration when a vehicle is nearby. The major benefit would be reduced risk of distraction.

\subsubsection{Improve Nearby Drivers' Awareness}

Accident data shows that proximity errors committed by car drivers are a factor in most side and rear heavy truck accidents with cars. In order to address this, a truck-based sensing system could be used to trigger exterior warnings to the surrounding vehicles. All of these systems would require very quick sensing and a simple, easily recognized message to have any significant effect. These countermeasures also rely on the drivers (in this case, of the nearby vehicles) to take an appropriate action when warned of danger.

\section{Visual Warnings}

Exterior visual warnings could include flashing lights, flags, or pop-out signs. When the sensing system detects an object approaching into a danger zone, one of these warnings could be used to 
alert the other driver. If a visual signal could be triggered fast enough and were easily recognized (like a flashing light), then proximity accidents might be reduced.

\section{Audible Warnings}

Horns or loudspeakers could be added on the rear and sides of the trailer to project a simple warning message if a vehicle approaches too close.

\section{Truck/Car Communication}

Vehicle-to-Vehicle and Vehicle-to-Infrastructure communication has been discussed for many years. If these systems are implemented, a truck-based sensing system could be used to directly inform nearby vehicles if they are entering a danger zone, and rely on in-vehicle warnings to notify each driver.

\subsubsection{Preventing Risky Actions}

Although it is important to keep the human in the loop, under certain conditions you cannot rely on the vehicle operator to take appropriate actions. If distracted or confused about the situation, drivers may even take actions that exacerbate dangerous situations. To prevent such actions, it may be possible to put passive/active countermeasures in place. These would be countermeasures that do nothing except to resist or prevent a poor motion (e.g. turning into a lane with another vehicle inside) by the driver.

\section{Haptic Warning}

One easy way to convey information is through the steering wheel. If a driver attempts to move the wheel in a direction that would move the truck to close to a neighboring vehicle, the wheel could vibrate or push back. The driver would still be able to make the maneuver, but would receive some negative feedback about it. 


\section{Resistive force}

One step beyond steering wheel motion is a resistive force against the motion. If the turn creates a dangerous situation, progressively higher turning resistance could be applied. This would likely work best when combined with another warning system, so the driver doesn't just push harder to overcome the obstacle. As with motion, this system would still allow the driver to make the decision.

\section{Audible or Visual Warnings}

A visual or audible warning could be used to notify the driver when his/her actions are risky. This would be like the proximity warning mentioned in 3.4 .10 above, but would only be used when the driver was taking such an action. In other words, they could be less intrusive than proximity warnings.

\subsubsection{Taking Preventative Actions}

If other countermeasures are unsuccessful at correcting a dangerous situation, it may be necessary for the safety system to take unilateral action. For rear and side impacts, these actions may include minor steering corrections and small accelerations or decelerations.

Steer

If a truck and nearby object are approaching rapidly and the driver has not taken corrective action, the vehicle steering system would be engaged in an attempt to widen the gap between the two vehicles. This action could only be taken if the safety system has 360-degree awareness of the truck's surroundings. In addition, it should be taken in concert with some braking, so that the maneuver doesn't move the truck outside of its own lane. 


\section{Brake}

If a vehicle is approaching the front side of the truck, an appropriate action may be light deceleration. This would allow the other vehicle to move past the truck in the front, preventing an impact.

Accelerate

If a vehicle is approaching the rear or rear side of the truck, a light acceleration may enable the two vehicles to avoid an impact. As with steering, however, this action should only be considered if the safety system has 360-degree situational awareness. 


\section{CONCLUSIONS}

A thorough literature was conducted to identify state-of-the-art crash avoidance system technologies and opportunities to reduce the cost of current crash avoidance systems for large trucks. A sensor evaluation was conducted and several technologies were identified for this system: magnetic sensors, ultrasonic sensors, vision system, and radar.

The magnetic sensor was identified as a cost reducing technology for crash safety; however, it has primarily been developed for vehicle identification at intersections and electronic compasses, but not for vehicle type classification and real time crash avoidance. In this report, preliminary work was conducted in the form of 2-D analytical modeling of dipoles and experimental bench tests to corroborate the findings of previous studies. A 3-D analytical single dipole model was then developed to better represent the magnetic phenomenon of real-world objects. A detailed parameter study was conducted to better understand the magnetic behavior of 3-D dipole models and the insights gained from the exercise were used for model matching with the experimental data. Road tests were conducted to capture the 3-D magnetic behavior of vehicles. The single 3-D dipole model was then extended to incorporate multiple dipoles for capturing the complex magnetic footprints recorded from vehicles. Mathematical functions capable of both eliminating the sign dependency of magnetic signals and producing a magnitude threshold for the different vehicle types were developed. The analytical and experimental study thus conducted showed that vehicle magnetic behavior could indeed be captured by mathematical models and that a magnetic sensor could be used to identify vehicle types.

The magnetic sensor was identified as a suitable sensor for vehicle classification; however, due to the sensor's range dependency, sensor fusion is required with a range sensor. This report investigated the application of statistical algorithms in the form of a Bayesian filter to enhance vehicle identification that uses an ultrasonic sensor and a magnetic sensor combination. This 
research study utilized the knowledge gained by the authors in a previous study on the applicability of ultrasonic and magnetic sensor fusion for vehicle detection. This report presents a detailed description of the procedure to formulate a two step prediction/correction based Bayesian filtering algorithm for both the ultrasonic and magnetic sensors. Statistical sensor models were developed for each type of sensor and individually utilized in the Bayesian filter algorithm. The results obtained showed a reduction in process noise and sensor anomalies that negatively influence the credibility of vehicle detection (Section 3.3.2.). A joint Bayesian filter algorithm was then developed to facilitate sensor fusion. Typical results of the filter performance indicates that the filter performs at greater than $80 \%$ accuracy overall. The results obtained clearly show the ability of the probabilistic approach to further enhance the prediction of object detection and discrimination capabilities of an ultrasonic-magnetic sensor fusion system. This study shows that this filter is effective for systems such as blind spot detection and vehicle classification systems; however, further studies must be conducted for systems that require irreversible decision making such as airbag deployment or active braking. 


\section{REFERENCES}

[1] Federal Motor Carrier Safety Association (FMCSA), "Large Truck Crash Causation Study Analytical Users Manual," <http://ai.fmcsa.dot.gov/ltccs/data/documents/LTCCS_Manual_Public.pdf〉, June 2006.

[2] M. Kunert, G. Rollmann, H.-L. Bloecher, J. Schuermann, " 24 GHz ultra-wideband vehicular short range radar systems technology and regulatory aspects overview," International Wroclaw Symposium and Exhibition on Electromagnetic Compatibility, 2006, Wroclaw, pp. 28-30. June 2006

[3] K.M. Strohm, H.-L. Bloecher, R. Schneider, J. Wenger, "Development of future short range radar technology," Radar Conference, 2005. EURAD 2005. European , vol., no., pp. 165-168, 6-7 Oct. 2005

[4] R. Mende, A. Zander, "A multifunctional automotive short range radar system" Smartmicro GmbH 2000.

<http://www.smartmicro.de/GRS_2000_Multifunctional_Short_Range_Radar_Syst em.pdf $>$

[5] M. Kunert, G. Rollmann, H.-L. Bloecher, J. Schuermann, "24 GHz ultra-wideband vehicular short range radar systems technology and regulatory aspects overview," International Wroclaw Symposium and Exhibition on Electromagnetic Compatibility, 2006, Wroclaw, pp. 28-30. June 2006

[6] Sergio Damiani, "State-of-the-art of current Collision Avoidance Systems." REPOSIT: RElative POSitioning for collIsion avoidance sysTems. 9 Jan. 2008. 20 Feb. $2008<$ 〈ttp://www.ist-reposit.org>.

[7] A. Carullo, M. Parvis, "An ultrasonic sensor for distance measurement in automotive applications," Sensors Journal, IEEE , vol.1, no.2, pp.143-147, Aug 2001.

[8] Kai-Tai Song; Chih-Hao Chen; Cheng-Hsien Chiu Huang, "Design and experimental study of an ultrasonic sensor system for lateral collision avoidance at low speeds," Intelligent Vehicles Symposium, 2004 IEEE , vol., no., pp. 647-652, 14-17 June 2004.

[9] J.E. Lenz: "A review of magnetic sensors," Proceedings of the IEEE, vol.78, no.6, pp.973-989, Jun 1990

[10] Honeywell, "Three-axis Magnetic Sensor Hybrid." Honeywell Magnetic Sensors. Feb. 2004. 10 Apr. 2008 <http://www.magneticsensors.com/datasheets/hmc2003.pdf>.

[11] M. J. Caruso: "Applications of Magnetoresistive Sensors in Navigation Systems," Honeywell Inc. Apr. 2008 < http://www.magneticsensors.com/datasheets/sae.pdf>

[12] M. J. Caruso, L. S. Withanawasam: "Vehicle Detection and Compass Applications Using AMR Magnetic Sensors," Honeywell Inc. Apr. 2008 <http://www.magneticsensors.com/datasheets/amr.pdf> 
[13] S. Y. Cheung, S. C. Ergen, and P. Varaiya: "Traffic Surveillance with Wireless Magnetic Sensors," Paper \#4779, University of California Berkeley, CA. Apr. 2008 < http://paleale.eecs.berkeley.edu/ varaiya/papers_ps.dir/sensors_its_final9.pdf>

[14] R. Lao and D. Czajkowski: "Magnetoresistors for Automobile Detection and Traffic Control," SENSORS Mag., pp.70-73, April 1996

[15] T. Phan, B. W. Kwan, L. J. Tung: "Magnetoresistors for vehicle detection and identification," Systems, Man, and Cybernetics, 1997. 'Computational Cybernetics and Simulation'., 1997 IEEE International Conference on , vol.4, no., pp.3839-3843 vol.4, 12-15 Oct 1997

[16] "Application Note-AN218 Vehicle Detection Using AMR Sensors," Honeywell Magnetic Sensors. Aug. 2005. Apr. 2008 <http://www.magneticsensors.com/datasheets/an218.pdf>.

[17] A. Shaffer, G. Aulakh, A. Getman, and R. Miller: "Vehicle Blind Spot Detection Using Anisotropic Magnetic Resistive Sensors," 2005 SAE International.

[18] A. Shaffer, G. Aulakh, A. Getman, and R. Miller. "Vehicle Sensors and Actuators (Part 1 \& 2)." SAE 2005 World Congress \& Exhibition, Apr. 2005, Detroit, MI, USA. SAE International. Vol. 2005-01-0456. SAE International 2005. 53-58

[19] "Unibrain Fire-i digital camera." Unibrain - The FireWire (FireWire 800 - IEEE 1394b) Innovators. Mar.-Apr. 2008 <http://www.unibrain.com/Products/VisionImg/Fire_i_DC.htm>.

[20] MaxBotix, "LV-MaxSonar®-EZ4 ${ }^{\mathrm{TM}}$ High Performance Sonar Range Finder." MaxBotix ${ }^{\circledR}$ Inc. Jan. 2007. Feb. $2008<$ http://www.maxbotix.com/uploads/lvmaxsonar-ez4-datasheet.pdf $>$.

[21] Thurn, Sebastian, Wolfram Burgard, and Dieter Fox. Probabilistic Robotics. Cambridge: The MIT P, 2006.

[22] Pinheiro, Pedro, and Pedro Lima. Bayesian Sensor Fusion for Cooperative. Proc. of 8th Conference on Intelligent Autonomous Systems (IAS-8), 2004.

[23] Fox, Dieter, Jeffery Hightower, Henry Kaus, Lin Liao, and Donald J. Patterson. Bayesian Techniques for Location Estimation. Proc. of 2003 Workshop on Location-Aware Computing.

[24] Koch, Karl-Rudolf. Introduction to Bayesian Statistics. New York: Springer, 2007. 